\title{
Towards Personalized Interactomes
}

by

\author{
Allen Amos-Binks
}

A thesis submitted to the Faculty of Graduate and Postdoctoral Affairs in partial fulfillment of the requirements for the degree of

Master of Applied Science

in

Electrical and Computer Engineering

Carleton University

Ottawa, Ontario

(C) 2015, Allen Amos-Binks 


\begin{abstract}
This thesis presents a preliminary study to evaluate the ability of our sequence-based protein-protein interaction prediction tool (PIPE) to detect changes in the interactome caused by non-synonymous Single Nucleotide Polymorphisms (SNPs). High performance computing is used to explore the effect of SNPs on the interactome and results are mixed. Sequencebased PPI prediction is not sensitive to SNP-induced interactome changes, however sequence-based PPI interaction site prediction can be used to extract some information regarding interactome changes.

PIPE on its own does not perform well on detecting the effects of SNPs. However, experiments using a contemporary sequence-based method confirm that this lack of sensitivity is not limited to PIPE, but likely affects all sequence-based methods.

Using the interaction site prediction feature of PIPE, PIPE-Sites, a number of interactions are identified for which there is reason to believe that they may, in fact, be affected by SNPs. These interactions are identified using subsets of disease-causing SNPs.

To examine the effect of collections of co-occuring SNPs, genotypes are extracted from data arising from the 1000 Genomes initiative. For these genotypes, it appears that PIPE-Sites is able to identify subsets of interactions where SNPs are enriched and therefore likely affected by that particular genotype.
\end{abstract}




\section{Acknowledgements}

I would like acknowledge financial support from the Natural Sciences and Engineering Research Council of Canada (NSERC) and Compute Canada, specifically the High Performacne Computing Virtual Laboratory (HPCVL).

I would also like to acknowledge the invaluable expertise and support of Dr. Frank Dehne, Dr. James R. Green, Dr. Ashkan Golshani, Dr. Alex Wong, Dr. Sylvain Pitre, Dr. Riva Soucie, Adam Amos-Binks and Andrew Shoenrock.

Finally, I would like to thank Lucy Barnes for her encouragement and support during this proccess. 


\section{Contents}

1 Introduction $\quad 1$

1.1 Motivation ......................... 1

1.2 Research Goals . . . . . . . . . . . . . . . 2

1.3 Summary of Methodology . . . . . . . . . . . . . 2

2 Background 5

2.1 Significance of PPI Prediction . . . . . . . . . . 7

2.2 Significance of PPI Binding Site Prediction . . . . . . . . . . . 11

2.3 SNPs and their effect on PPIs ............... 12

2.4 Computation Tools that Predict SNP Effects . . . . . . . . . 15

2.5 The PIPE Algorithm . . . . . . . . . . . . . . . 15

2.5.1 Algorithm ..................... 16

2.5.2 Interaction Site Prediction . . . . . . . . . 19

2.6 Limitations of PIPE _..................... 21

3 Quanitfying Performance of sequence-based PPI Prediction on $\begin{array}{ll}\text { SNPs } & 25\end{array}$

3.1 Data Source: SKEMPI database ............. 25

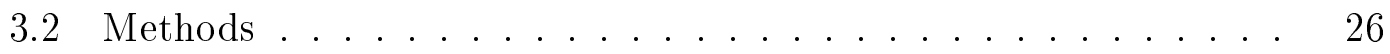

3.2.1 Data Conversion to PIPE Input . . . . . . . . . 26

3.2.2 Creating Mutated Protein Sequences from PDB Files . . . 30

3.2.3 Generating Input Pairs for Individual PIPE SNP Runs . . 31

3.2.4 Running PIPE on the M9000 Enterprise Server . . . . . 32

3.2.5 Run Time Information for M9000 PIPE Runs . . . . . . 35

3.2.6 Alternative PPI Prediction Methods . . . . . . . . 36 
3.3 Results for SKEMPI Data . . . . . . . . . . . . . . 36

3.3.1 PIPE Score Change for SKEMPI SNPs . . . . . . . . 37

3.3.2 PDB and Affinity Analysis of SKEMPI Data using PIPE . 39

3.3.3 Alternative Sequence-Based PPI Method Results using SKEMPI Data ........................ 41

3.4 Conclusion . . . . . . . . . . . . . . . . . 43

4 Using PIPE-Sites to Identify Interactome Changes Caused by Disease-associated SNPs $\quad 44$

4.1 Data Source: SNPdbe . . . . . . . . . . . . . . . . . . 44

4.1.1 SNPdbe PIPE Run Data . . . . . . . . . . . . . . . 45

4.2 Methods for PIPE-Sites SNPdbe Data Analysis . . . . . . . 46

4.2 .1 P Value Calculation . . . . . . . . . . . 47

4.2 .2 Per-Site Analysis of SNP Locations . . . . . . . . . 48

4.2.3 Per-Protein and Per-Interaction, Site Analysis of SNP Lo-

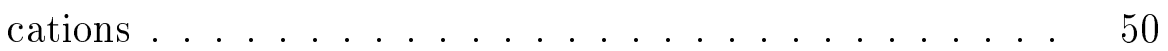

4.2.4 Per Disease Analysis . . . . . . . . . . . . . . 51

4.2 .5 Q Value Analysis . . . . . . . . . . . . . 54

4.2.6 Generating Inputs for Individual PIPE Runs of the SNPdbe Data ........................ 55

4.3 Results. . . . . . . . . . . . . . . . . 58

$4.3 .1 \quad$ Per Site Results . . . . . . . . . . . . . 58

4.3.2 Per Protein and Per Interaction Results _. . . . . . . 59

4.3 .3 Per Disease Results . . . . . . . . . . . . . . . . 60

4.3 .4 D3JS Graphs . . . . . . . . . . . . . . . 62

4.3.5 SNPdbe PIPE Score Results for PIPE-Sites Data . . . . . 64 


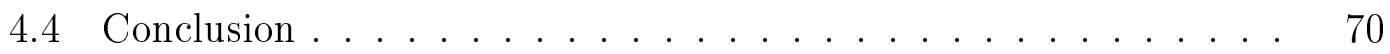

5 Using PIPE-Sites to Identify Interactome Changes Caused by Known Co-occurring SNPs from 1KG Genotypes 72

5.1 Data Source: 1000 Genomes . . . . . . . . . . . . 72

5.1 .1 Sequence Alignment ............. 73

5.1 .2 SNP Calling .................. 74

5.1.3 SNP Acquisition from $1 \mathrm{KG} \ldots \ldots . \ldots 75$

5.1.4 SNP Conversion to Amino Acid Substitutions . . . . . 75

5.2 PIPE Sites 1 KG Results . . . . . . . . . . . . . 77

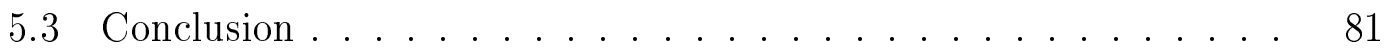

6 Conclusion $\quad 84$

6.1 Summary of Outcomes ................. 84

6.2 Future Work .................... 86

A Pseudo Code, Extraneous Data 95

A.1 Optimization of the PIPE Algorithm . . . . . . . . . . 95

A.2 Pseudo-Code for Input Generation _ . . . . . . . . . . . . 99

A.3 Generating Interaction Graphs for Individual PIPE Runs . . . . . 103

A.4 SKEMPI PIPE Run Analysis Files . . . . . . . . . . . 104

A.5 Per Protein Interaction Site Analysis of SNP Locations . . . . . 105

A.5.1 Per Protein Results . . . . . . . . . . . . 106

A.6 Per Interaction, Interaction Site Analysis of SNP Locations . . . . 107

A.6.1 Per Interaction Analysis Results . . . . . . . . . 108

A.7 Significant Interactions at a Q-Value of 0.001 . . . . . . . . 109 


\section{List of Tables}

3.1 Amino Acid Conversion Table . . . . . . . . . . . 29

3.2 M9000 Run Time Information . . . . . . . . . . . . . 35

4.1 Disease SNPs from PIPE Benchmark Run . . . . . . . . . . 46

4.2 Q-Value Results . . . . . . . . . . . . 61

4.3 PIPE Sites Score Change Statistics - All Mutations. Where 'n' is the number of interactions . . . . . . . . . . . 67

4.4 PIPE Sites Score Change Statistics - C to W Mutations. Where 'n' is the number of interactions ............. 68

4.5 Z Value Mean Comparison for Score Change of All SNPdbe Mutations 70

5.1 ANNOVAR Output File . . . . . . . . . . . 76

5.2 1KG Sample Data Summary 1 . . . . . . . . . . . . 78

5.3 1KG Sample Data Summary 1 ............. 79

5.4 1KG PIPE-Sites Analysis Results Summary 1 . . . . . . . . 82

5.5 1KG PIPE-Sites Analysis Results Summary 2 . . . . . . . 83

6.1 Summary of Outcomes and Applications . . . . . . . . . 86

A.1 Example PIPE Analysis File of a SNP . . . . . . . . . . . . 104 


\section{List of Figures}

2.1 Protein Interaction with Interaction Site . . . . . . . . 6

2.2 SNP Causing a Protein Sequence Change . . . . . . . . . . 7

2.3 PIPE Algorithm Overview [1] ............... 16

2.4 PIPE Score Surface Plot for a Prediction Pair . . . . . . . . . 18

2.5 PIPE Score Contour Plot for a Prediction Pair . . . . . . . . . . 19

2.6 PIPE Score Surface Plot Showing More Than Three Peaks . . . . 22

2.7 PIPE Score Contour Plot Showing More Than Three Peaks . . . . 23

3.1 Chains from a PDB Entry .............. 31

3.2 M9000 Enterprise Server Hardware Architecture . . . . . . . . . . 34

3.3 Histogram of Absolute PIPE Score Changes . . . . . . . . . . 38

3.4 PIPE Score Change vs. Protein Affinity Change for SKEMPI SNPs 40

3.5 sig_prod Score Change vs. Protein Affinity Change for SKEMPI

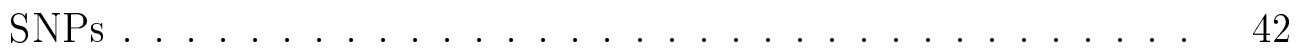

4.1 Per Site Interaction P-Value Analysis . . . . . . . . . . . 49

4.2 Per Protein Per Interaction Analysis . . . . . . . . . . . . 50

4.3 Per Disease P-Value Analysis . . . . . . . . . . . . . 52

4.4 Randomly Generated Interaction Site . . . . . . . . . . . 53

4.5 Workflow of a PIPE Run for one SNP . . . . . . . . . 57

4.6 Per Site Analysis Histogram . . . . . . . . . . . . 58

4.7 Per Protein, Per Interaction Analysis Histogram . . . . . . . . . 60

4.8 D3 Web Graph of Interactions with $\mathrm{Q}<0.05 \ldots \ldots 65$

4.9 D2 Web Graph with Additional Information Shown after a Transition 66

5.1 SNP Prediction from Multiple DNA Sequence Reads . . . . . . 74

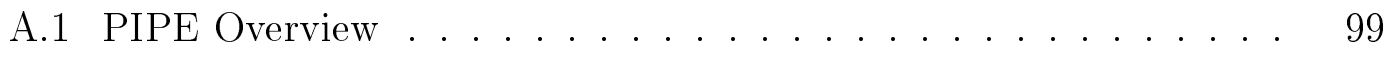


A.2 Per Protein Interaction P-Value Analysis . . . . . . . . 106

A.3 Per Interaction P-Value Analysis _ . . . . . . . . . . 107

A.4 Per Interaction Analysis Histogram of P-values . . . . . . . . 108 


\section{List of Algorithms}

1 Gentab Pseudo Code . . . . . . . . . . . . . . 28

2 PIPE Scheduler[1] ..................... 97

$3 \quad$ PIPE Worker[1] . . . . . . . . . . . . . . . 98

4 create_sets_against_all_input.py Pseudo Code ........ 100

5 create_pairs_from_SNPs.py Pseudo Code . . . . . . . . . . 101

6 shell_gen.py Pseudo Code . . . . . . . . . . . . . 102 


\section{Introduction}

\subsection{Motivation}

High-throughput next-generation sequencing techniques will soon make it feasible to sequence the genome of an individual as part of typical patient care, thereby elucidating the multitude of genetic variants that make each person unique [2]. These genetic variants will provide the information needed to give patients truly personalized care based on their unique DNA. One step towards this goal is the prediction of the effect these genetic variants will have on the patient's proteinprotein interaction network, hereafter referred to as the interactome.

Predicting the effect of Single Nucleotide Polymorphisms (SNPs) on proteinprotein interactions (PPIs) has received little attention thus far in literature, largely due to a lack of experimental data linking SNPs with changes to the interactome. Since PPIs are known to mediate critical functions within the cell, and are often drug targets [3], developing the ability to predict an individual's interactome, based on the patient's set of SNPs, would be a major step towards personalized medicine. To this end, identifying interactions where it appears SNPs are enriched would provide valuable insight into which interactions are most likely affected most by a specific set of mutations, whether they are sets of disease related SNPs or less harmful SNPs that co-occur in nature.

While several sequence-based methods of PPI prediction are available, it is unclear whether such methods are sufficiently sensitive to changes in protein sequence to detect the impact of non-synonymous SNPs on PPIs. Exploring and quantifying this ability is the central aim of this thesis, as this question has not been studied, to the best of our knowledge. Furthermore, the secondary goal of identifying PPIs that are most likely to be impacted by SNPs would provide valu- 
able insight into the effects specific sets of SNPs have on the interactome, whether those SNPs are disease related such as those in [4] or SNPs that are known to co-occur such as those from [5].

\subsection{Research Goals}

The research contained herein focuses on three research outcomes in an effort to provide quantitative results regarding the effect of SNPs on the human interactome.

The desired outcomes of this research are:

1. Quantifying the performance of computational sequence-based PPI prediction methods in gauging the effect of SNPs on the interactome

2. Compare computationally-predicted PPI interaction sites with known diseasecausing SNP locations with two aims:

(a) Identify potential PPIs affected by congenital diseases

(b) Identify diseases whose mechanism of action may be to disrupt PPIs

3. Given that not all known SNPs will co-occur in a single individual, investigate the effect of genotypes (i.e. collections of co-occurring SNPs) on the interactome

\subsection{Summary of Methodology}

In order to achieve the research goals detailed in Section 1.2, the Protein Interaction Prediction Engine (PIPE) [1], summarized in Section 2.5, was used to provide high confidence PPI predictions as well as PPI site predictions, via PIPE-Sites [6], 
on an interactome scale. Using the PPI prediction score generated from PIPE as well as the interaction site predictions, experimentally verified mutations from the Structural database of Kinetics and Energetics of Mutant Protein Interactions (SKEMPI) [7], SNPdbe [4] and the 1000 Genomes Project (1KG) [5] were analyzed in a number of different ways to quantify the effect SNPs have on PPI predictions. These datasets are described in Sections 4.1, 5.1 and 3.1 respectively.

Section 3 addresses the first research goal by examining the performance of PIPE and another PPI prediction method, sig_prod from [8], in predicting SNPinduced changes to the interactome. For PIPE, this performance was quantified as the change in PIPE score caused by SNPs. This analysis was performed using data from the SKEMPI project, which provided SNPs and associated information on the kinetic changes induced by these SNPs. SKEMPI was used because the kinetic information contained therein provided a direct metric by which PIPE score change could be evaluate against. The results from the score change analysis can be found in Section 3.3.

Section 4 investigates the second research goal by using the interaction site prediction feature of PIPE. Using the predicted interaction sites for all interactions from PIPE two main experiments were performed:

1. Quantifying enrichment of SNPs at specific interaction sites

2. PIPE score change analysis between the cases when a SNP falls inside or outside of an interaction site

These results and the accompanying methods for the PIPE score change when considering the interaction site are found in Section 4 .

The third research goal was addressed in Section 5 and investigated using PIPE predicted interaction sites in conjunction with collections of SNPs (geno- 
types) obtained from the $1 \mathrm{KG}$ project. Using collections of SNPs from a subset of individuals, the locations of these SNPs were examined for enrichment at predicted PPI sites. 


\section{Background}

Proteins are major building blocks on which all biological beings rely for a variety of functions, including DNA replication, catalyzing metabolic processes, biological process regulation and many more [3]. Proteins themselves are chains of amino acids that form $3 \mathrm{D}$ structures and then perform their biological function [3].

While performing their biological functions, proteins rarely act alone, they often interact with other proteins to form complexes, which in turn perform the desired function [3]. These interactions are, therefore, integral to the proper function of biological pathways in nature. When these protein interactions occur, the interaction partners do not completely convolve, rather, parts of each protein come together like a lock and key [3]. These areas that form the "lock and key" are the interaction sites of the proteins. Figure 2.1 shows an example of two proteins with the interaction sites highlighted in green. As can be seen, the interaction site shapes are complimentary to one another, facilitating the interaction between these proteins [3]. Furthermore, the physicochemical properties exhibited on the protein surfaces at the interaction site are often complementary to each other, which further facilitate interaction [3].

The sequence of amino acids used to build proteins is coded in DNA within genes. Each DNA sequence is made up of a series of one of four bases, Adenine(A), Thymine(T), Cysteine(C) and Guanine(G). Each of 20 naturally occurring amino acids in human cells is coded for by DNA subsequences of 3 bases, known as codons. For example for the amino acid alanine, the DNA sequence is coded for by the subsequences: GCT, GCC, GCA, GCG. The sequence of codons that codes for a protein is known as a gene.

Genetic variation can be caused by a number of changes to the DNA. Shorter 

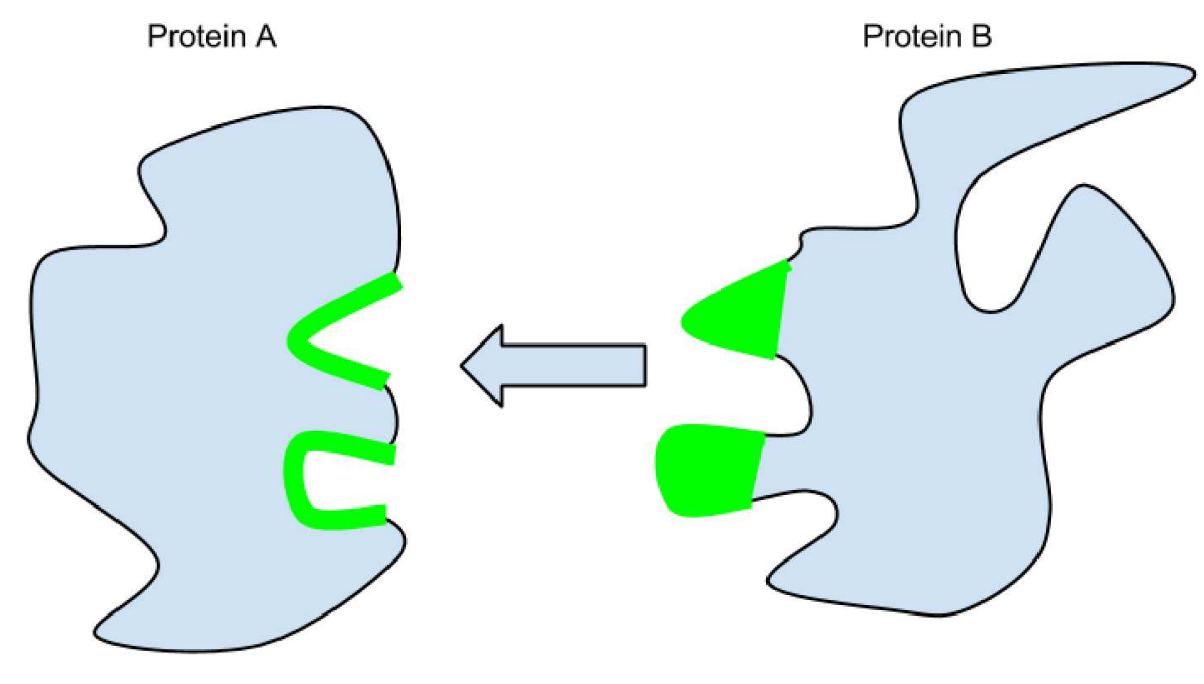

Figure 2.1: Protein Interaction with Interaction Site

genetic variations include insertion-deletions, where parts of the DNA code for proteins are added or removed, and SNPs, where one base is changed in the DNA code. Longer genetic variations include the complete loss of a gene, copying of a gene multiple times throughout the genome and translocations, where genes are moved around in the genome.

Mutations outside of the coding region for a protein affect other factors like gene expression, thereby affecting the abundance of the protein.

SNPs do not always cause a change in amino acid sequence, for example a change in a codon from GCT to GCC would still result in the same protein sequence since both codons represent the amino acid alanine. All SNPs discussed in this thesis are non-synonymous SNPs, which are SNPs that do cause an amino acid change in the protein sequence. Of these non-synonymous SNPs, it is estimated that a person has, on average, about 108,000 , though this number is highly variable [5]. Figure 2.2 shows one example of a non-synonymous SNP, where a SNP changes the fourth codon from GCT (coding for alanine) to ACT (coding 


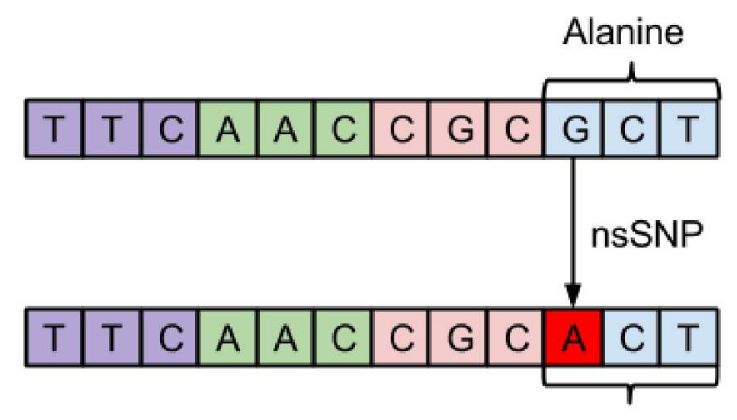

Threonine

Figure 2.2: SNP Causing a Protein Sequence Change

for threonine) [3].

It is easy to visualize the genome as one long sequence of DNA bases, however human cells each have two copies of genetic code, one from each parent. This complicates gene sequencing since both copies are mixed together when samples are sequenced. These two copies of the genome are referred to as the genotype. In each genotype there are subsets of genes that are inherited together. These collections of genes that co-occur and are inherited together are known as haplotypes. Haplotypes can be inferred through statistical means; this is known as haplotype imputation and is beyond the scope of this thesis. For the purposes here, genotype information was extracted in precomputed form from the $1 \mathrm{KG}$ data.

\subsection{Significance of PPI Prediction}

Since PPIs are an integral part to biological pathways that mediate processes in living organisms, understanding how they function is critical to understanding how living organisms function in normal and adverse conditions [6, 9]. In total for the 22,000 human proteins, there are approximately 242 million possible protein interactions, therefore computational tools are necessary to focus the efforts of 
wet lab experiments [10].

There is estimated to be approximately 650,000 total PPIs comprising the human interactome [10]. At this time only approximately 40,000 are experimentally verified [11], representing only a small fraction of the potential human interactome. In the past, PPI verification was done using single pairs of proteins being tested for interaction against one another, however these studies are too slow and provide too low throughput for interactome-wide studies.

Recent studies use higher throughput methods of PPI verification such as Yeast Two Hybrid and TAP-Tagging, which can be used to discover hundreds of new interactions in one experiment, such as those found in [12]. Though this number is high relative to past experimental techniques, the results are not always of high quality and the experiments themselves are expensive in terms of time and resources $[13,12]$.

In order to optimize the use of resources and augment experimental verification efforts, computational methods for predicting PPIs with high confidence are desirable. PPI predictions from computational methods, if reported with high confidence, can be used to direct the research efforts of the scientific community. There are multiple computational methods available that predict PPIs using various approaches, each with distinct advantages and disadvantages.

One popular method for predicting PPIs uses the 3D structure of proteins in question. For the purposes of interactome-wide studies, these structure based methods have some distinct disadvantages. Firstly, only $39 \%$ of the human proteome can be assigned 3D structures [11]. Considering that the structure of both query proteins in a putative interaction must be known, this results in only $16 \%$ of protein pairs being amenable to these structure-based techniques [11]. Structurebased methods are also computationally expensive and are normally applied on a 
pair by pair basis, not on the interactome scale [13]. One structure-based method from [14] was applied to 36 million of the possible 242 million PPIs, however the corresponding precision and recall associated with this experiment was $41.2 \%$ and $7 \%$ respectively. Due to the magnitude of the interactome, this means the predictions made likely contain an unreasonably high number of false positives, which makes these predictions unsuitable for directing wet-lab experiments.

Sequence-based methods offer an alternative to the computationally expensive structure-based methods and overcome some of the limitations of structure-based methods. Some of these methods have been reviewed in depth in [15]. However, many of these sequence-based methods still suffer from performance issues when scaled to make interactome-wide predictions [13, 12]. As discussed below, significant efforts in algorithmic optimizations and parallelization have enabled PIPE to be the sole method applicable at the genome scale [1].

Genomic methods of PPI prediction use the information generated from genome sequencing technology [13]. The main feature for these methods is the co-location of genes that code PPI interaction partners. These methods are able to predict certain kinds of interactions however there are drawbacks that make this method unsuitable to human interactome studies. This approach is not able to predict interactions between genes that are not co-located together. This class of methods also produces a sizable number of false positives since many co-located genes code for proteins that do not in fact interact.

Evolutionary PPI prediction methods look at the evolutionary characteristics of a protein across multiple organisms. If proteins are related functionally, it stands to reason that they may have similar interaction partners. While useful, these methods require extensive homology information, which is not available for many proteins in the human interactome. These methods are also unable to 
identify species-specific PPIs.

Structure based prediction methods, similar to evolutionary PPI methods, search for homologs of the proteins in an interaction database or compare parts of the protein with known interaction interfaces from known 3D structures. As mentioned previously, these methods suffer from a lack of experimentally verified data as there are relatively few solved 3D structures, therefore these methods cannot be applied to arbitrary protein pairs since most pairs do not have a known structure for both proteins. These methods are also computationally expensive and few can be run quickly on the interactome scale. Both these factors make these methods unsuitable for an interactome-wide analysis.

Another PPI prediction class of method is based on domain conservation, where a domain is an independent, functional area of a protein. In these methods the proteins in question have their own domains compared with those in a known domain interaction database [13]. This class of methods suffers from limited accuracy since, in general, protein domains comprise large portions of the protein. Since larger protein regions are examined, the sensitivity to smaller changes between interaction types limits the applicability of these methods to the interactome.

In this thesis, we will make use of a highly optimized, computationally efficient sequence-based PPI prediction method developed by our research group here at Carleton University. This method is called the Protein Interaction Prediction Engine (PIPE) and it was used to make fast, high confidence PPI predictions on the interactome.

There are also other sequence-based PPI prediction methods, like PIPE that use short polypeptide chains as the main feature to predict PPIs. Of these methods, four were compared in [15] that do not require homology data. In this com- 
parison, the four methods were compared against validation sets from both yeast and human interactomes and it was found that PIPE "significantly outperforms the others in terms of recall-precision across both the yeast and the human data" [15].

\subsection{Significance of PPI Binding Site Prediction}

Aside from predicting PPIs themselves, it is also important to identify the area in each protein which facilitates the bond between the interaction partners. In Figure 2.1 this area is highlighted in green.

Protein binding site identification is important in identifying critical motifs involved in protein interactions, biological pathways and protein function [6]. Knowing the binding site of a protein or for a specific interaction is the first step in understanding the potential interactions any SNPs will impact.

As with PPI predictions, computational methods for binding site prediction offer significant advantages in terms of cost and time effectiveness compared with experimental techniques. Similarly, as with PPI prediction there are 3D structural approaches that use docking software to predict binding sites, however as mentioned before these methods require 3D structure data which is not always available [6].

Sequence-based methods for binding site prediction overcome these limitations and provide valuable binding site prediction data. The majority of these sequencebased methods for binding site prediction rely on manually curated datasets of binding site motifs, which are difficult to experimentally verify and therefore do not result in particularly high quality training data [6].

This thesis uses PIPE-Sites, an extension of PIPE that provides the top three 
potential interaction sites (when available) based solely on sequence. These top three interactions sites represent three peaks in the PIPE interaction graph, which is discussed in Section 2.5.2. Similar to PIPE, since PIPE-Sites is a sequence-based method, it is able to provide fast high-confidence binding site predictions on the interactome scale [6].

PIPE-Sites, discussed in depth in Section 2.5.2, provides interaction site prediction by sorting the various peaks in the PIPE landscape that is generated for a pair of proteins. Each peak is sorted and classified and the three highest peaks are then reported to the user [6]. PIPE-Sites is able to predict the location of interaction sites, within $10 \%$ of the protein length, 6 times better than a randomly generated site, based on the binding sites from DOMINO used in [6].

\subsection{SNPs and their effect on PPIs}

The reference genome sequence from the Human Genome project represents a consensus genetic reference for which biological comparisons can be made, however it does not capture SNPs that cause the genetic variations seen between individuals. When an individual is sequenced, the individual's sequenced genome is compared to the reference genome to identify any genetic variation. The $1 \mathrm{KG}$ project found that on average a person has 3.60 million SNPs, as many as $3 \%$ of which cause

changes to the protein sequence [5]. It is important to note that SNPs are closely related to Single Nucleotide Variants (SNVs). SNVs are also genetic variations at a single base location in DNA. However, unlike SNPs, SNVs have less supporting statistical evidence, whereas SNPs are mutations that can be observed in any individual across a population.

There has been a large amount of analysis and verification of PPIs and interac- 
tion sites using the reference genome, however there has been comparatively little research into the effect of genetic variation on the human interactome [9]. While there has been little investigation so far, preliminary experiments show that even small changes, such as SNPs, can cause changes to a protein's physical interactions [16]. The results collected in the SKEMPI dataset further support the hypothesis that genetic variation can have a meaningful impact on the PPIs [7].

One study which examines the effect of SNPs on the interactome is [16]. In this study the effects of different types of mutations are examined to quantify their effect on the interactome of the mutation targets. The relevant conclusions from this study show that SNPs typically produce edgetic perturbations (i.e. the loss or gain of individual PPIs) in their mutation targets, rather than complete loss of all interactions. While the sample size is small, 5 protein's interactomes and 29 SNPs were examined, it was found that the majority of the SNPs caused edgetic perturbations or no change to the mutated proteins interactome. Only 5 of the 29 SNPs caused a complete interactome loss to their mutation targets. This result provides evidence that SNPs cause changes to the interactome, rather than completely disrupting protein interaction.

Other studies look at specific diseases caused by SNPs, such as the study found in [17], however this looks more at evolutionary variation at the genome level, not the effect these mutations have on the interactome.

There have been other studies that show certain diseases have a marked effect on PPIs. For example [18] found that two SNPs could account for increased susceptibility to colorectal adenomas due to a change in the interactome from these mutations.

Other examples of disorders that have been shown to be affected by SNPs causing interactome changes include, Meesmann Corneal Dystrophy [19], autoimmune 
lympho proliferative syndrome (ALPS) [20] and Liddle's Syndrome [21].

With the increasing availability of public data documenting the genetic variation of individuals, it has become more feasible to analyze and begin quantifying the changes that such genetic variation will have on PPIs. Some databases worth noting are the 1000 Genomes Project (1KG) [5], which seeks to identify all SNPs in at least 1000 individual genomes, SNPdbe [4], which catalogs known SNPs and any known association with disease or other phenotype, and SKEMPI [7], which catalogs and quantifies a large number of mutation-induced changes in PPI binding affinity. Each of these will be discussed in more detail in Sections 5.1, 4.1 and 3.1 , respectively.

SNPs that cause changes to amino acids that are buried within a protein structure often affect the function of that protein. However these effects are of less interest when examining the interactome, since SNPs on the surface of proteins, particularly those that occur in an interaction interface, potentially affect the protein interactome with more consequence [9]. Conversely, changes to the protein's core may disrupt scaffold structure necessary to form the PPI interface. It has also been shown in previous research that SNPs are enriched at protein interaction locations for some diseases [9].

It should be noted that the location of SNPs can be effected by biochemical properties inherant in DNA. For this reason and other evolutionary reasons, the distribution of SNPs is not completely random [22]. The analysis presented in this thesis only examines the location of SNPs and not their possible biochemical association. 


\subsection{Computation Tools that Predict SNP Effects}

Computational tools that predict the effect of SNPs have been introduced, such as those found in [23], [24] and [25]. These methods essentially fall into two categories: methods that use 3D structure homology data and methods that only use amino acid sequence. None of these methods specifically predicts interactome changes, rather they focus on predicting a functional change to the protein.

The SIFT algorithm from [24] predicts whether or not an amino acid substitution affects protein function and does not consider the protein in question's interaction partners. This method is based on the evolutionary conservation of amino acids. its underlying theory is that if an amino acid has been highly conserved through evolution, it is less tolerant to a substitution.

The method presented in [23] gives predictions on binding affinity based on classifiers from the SKEMPI database. Since this method uses 3D structure data to make these predictions, it would not be feasible to perform an interactome-wide analysis due to run-time issues and the lack of 3D structure data available.

It does not appear that there have been any interactome-wide studies into the affect SNPs have on protein interactions, however there is sufficient data to begin a pilot study using methods that can perform interactome-wide interaction analysis on the affect of SNPs, using tools such as PIPE.

\subsection{The PIPE Algorithm}

PIPE, developed at Carleton University, is a sequence-based PPI and binding site prediction tool that can produce high confidence PPI predictions at a False Positive Rate (FPR) of less than $0.05 \%$. PIPE predictions are based on a continuous score between zero and one. A higher PIPE score indicates an increased likelihood 


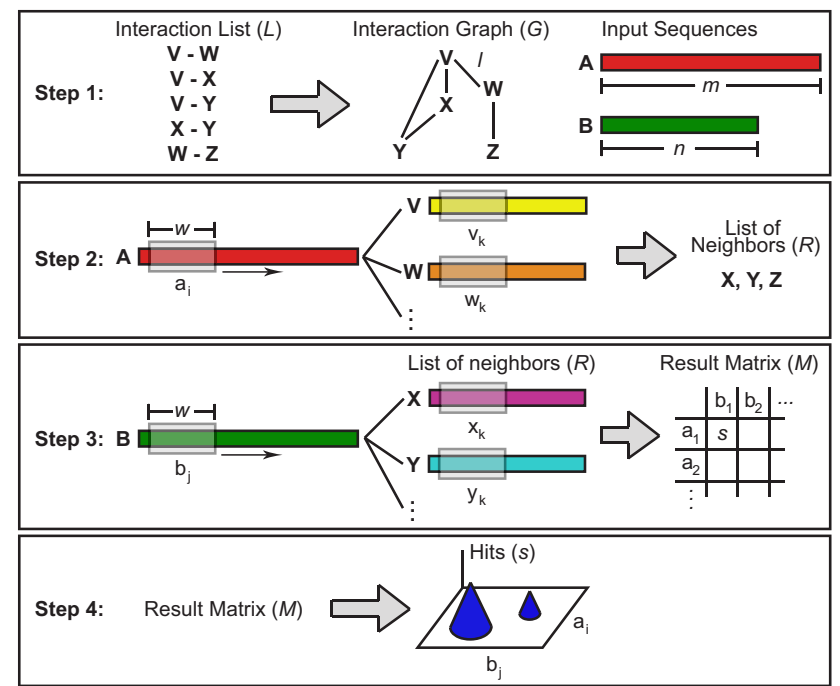

Figure 2.3: PIPE Algorithm Overview [1]

that two proteins interact.

\subsubsection{Algorithm}

The PIPE algorithm makes a prediction on whether two protein partners will interact based on the query protein sequences and an interaction database of experimentally verified protein interactions. This database of verified protein interactions is known as the interaction graph. The interaction graph $(\mathrm{G})$ has vertices $(\mathrm{V})$ that represent proteins and edges $(\mathrm{E})$ that represent experimentally verified interactions between the connected vertices. Figure 2.3 summarizes the basic PIPE algorithm.

In the first step of the algorithm, the interaction graph is created using the list of experimentally verified protein interactions.

In the second step, protein $A$ is divided into an array of uniformly sized, overlapping, protein windows or fragments, $a_{i}$, of size $w$, where $0 \leq i \leq|A|-w+1$. 
Using these fragments, the list of neighbors, $R$, is constructed. To accomplish this all proteins that have a fragment similar to $a_{i}$ are found. All known interactions with this subset of proteins containing similar fragments to $a_{i}$ are then placed in the list of neighbors, $R$. The sliding window used in the PIPE runs from this thesis was always set to 20 as increasing or decreasing the size did not result in any performance improvements [1].

To determine similarity between protein fragments a score is generated using the PAM120 matrix, which represents the probability of changes between amino acids through natural selection. For each corresponding amino acid pair, $x$ and $y$, a score based on the PAM120 matrix is assigned representing the probability that $x$ has been replaced by $y$ through natural selection. If the sum of all PAM scores for the window in question are above the specified threshold, the windows are deemed similar and the protein in question's neighbors are added to $R$.

In the third step, protein $B$ is examined using overlapping windows $b_{i}$ where $0 \leq i \leq|B|-w+1$. Each fragment is then compared to all fragments from all proteins in the neighbor list $R$ in a search for similar fragments, once again using the PAM120 score as a threshold for similarity.

The results matrix is an $|A| \mathrm{x}|B|$ matrix initialized to zeroes. Each row, i, represents a protein fragment $a_{i}$ and each column, $\mathrm{j}$, represents a protein fragment $b_{j}$. The result for a cell indicates how often the fragments $a_{i}$ and $b_{j}$ appear in proteins that are known to interact. Once all cells in the results matrix have been computed, the mean value of all cells is calculated, the result of which is known as the PIPE score. If the calculated mean is above a threshold, the proteins are predicted to interact.

The PIPE threshold is calculated using Leave-One-Out (LOO) cross-validation. In LOO testing, a positive pair is held out from the interaction graph. The PIPE 


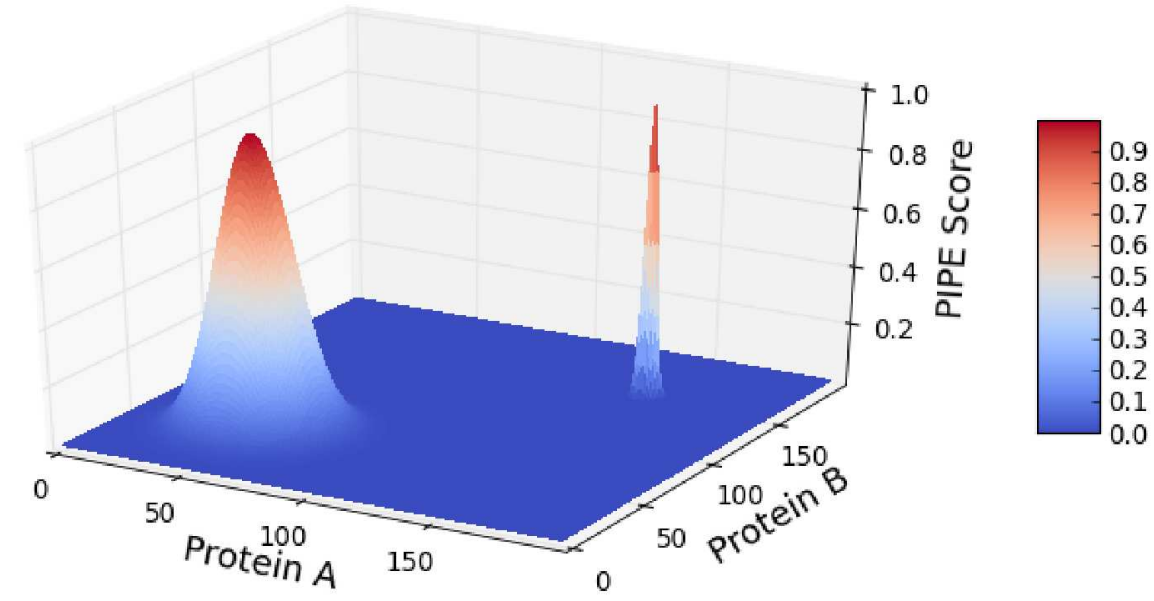

Figure 2.4: PIPE Score Surface Plot for a Prediction Pair

algorithm is then run and predictions made on the remaining positive pairs as well as a negative set of 100,000 pairs created using the techniques described in [26]. Using the sensitivity and specificity based on various PIPE thresholds, the desired operation point can be selected. In this thesis, the threshold selected corresponds to a specificity of 0.9994 and a sensitivity of 0.2376 .

The results matrix is a "landscape" of peaks and valleys that is used further for PIPE-Sites, described in Section 2.5.2. Figures 2.4 and 2.5 show examples of a $3 \mathrm{D}$ results matrix and the contour map from that results matrix.

Figure 2.4 shows an theoretical 3D contour plot of a possible PIPE landscape for two proteins. Figure 2.5 shows the same plot as a contour diagram. Protein $A$ is shown along the $x$ axis and Protein $B$ is shown along the $y$ axis. The number of hits from the interaction graph would be measured along the $z$ axis. In this case, two distinct peaks are shown. If the normalized score, calculated as discussed previously, is above the calculate PIPE threshold, these two proteins would be predicted to interact. 


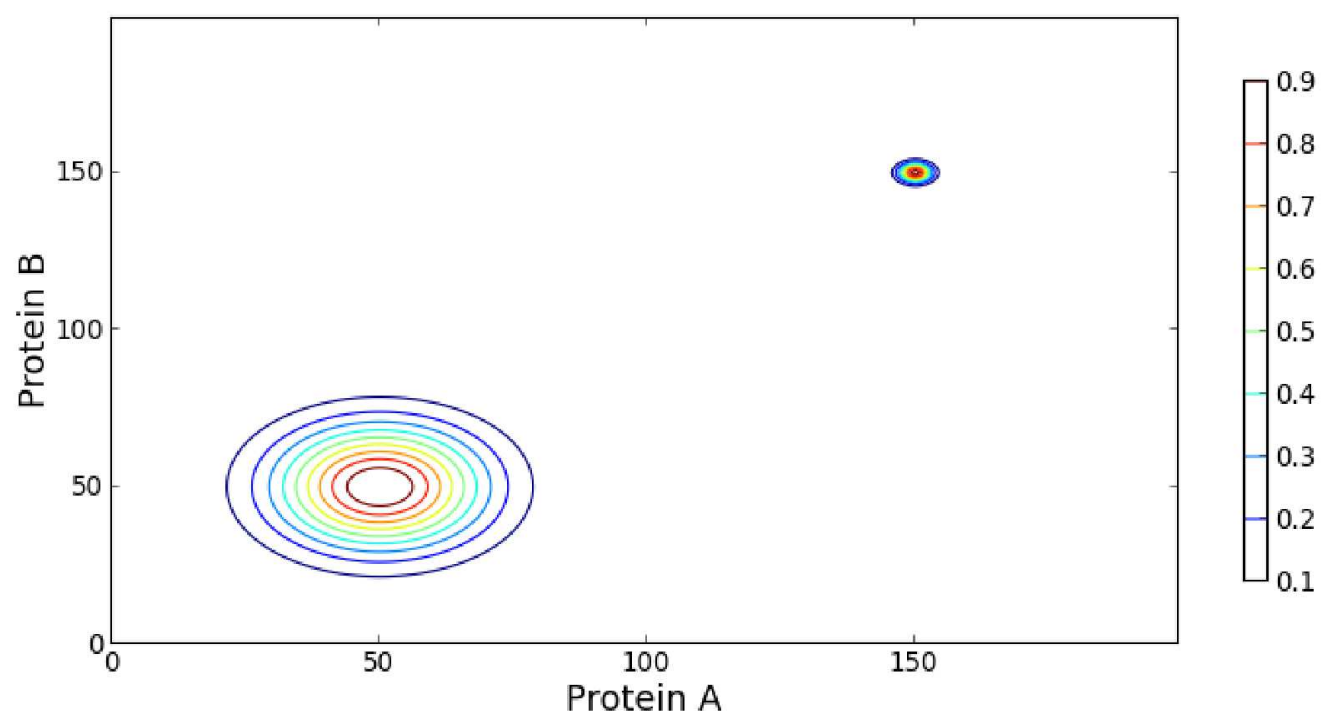

Figure 2.5: PIPE Score Contour Plot for a Prediction Pair

Due to the nature of the PIPE algorithm, frequently occurring protein fragments can have a detrimental effect on the specificity. If certain fragments are popular, not just in the interacting proteins but in the interactome overall, a protein pairs' score can be artificially inflated. To counteract this effect a computationally simple median filter is applied to the results matrix. For each cell, if the surrounding cells contain more zeroes than ones, the cell is set to zero, otherwise it is set to one. After this filter is applied, the final PIPE score is calculated [1].

\subsubsection{Interaction Site Prediction}

Simply predicting whether a pair of proteins will interact is useful, however it is also desirable to predict the binding sites for each interaction. Identifying these binding sites is the first step in modulating the protein interactions and the pathways these proteins are involved in, which is particularly important in drug design [6]. The PIPE score is an overall measure of interaction potential for the whole protein, however the resulting landscape produced by the PIPE algorithm 
can be examined to identify binding sites. The PIPE landscape consists of a number of peaks, where each peak and its surrounding area represent subsequences that are prevalent in interaction partners. For some interaction partners these peaks are more pronounced, as can be seen in Figure 2.4, which shows an example of two possible PIPE score peaks. A peak in the PIPE landscape represents an interaction site and the surrounding hill represents the coverage of the site on the respective proteins. Figure 2.5 shows the contour graph of the peaks from the 3D figure.

The interaction site itself is identified by examining each peak and the surrounding area. The maximum height of the peak, $\left|p_{i, j}\right|$, is first identified where $\mathrm{i}$ and $\mathrm{j}$ are locations in the PIPE results matrix. The PIPE site itself is initially only the single point of the peak at $(i, j)$. The height of the surrounding area is then examined by a walk in each direction from the peak to locations $\left|p_{i+1, j}\right|$, $\left|p_{i-1, j}\right|,\left|p_{i, j+1}\right|$ and $\left|p_{i, j-1}\right|$ which are compared to the preset cutoff percentage of peak height, percentPeak. If a point along the walk is below the percentPeak threshold value, $\left|p_{i-n, j-m}\right| \leq$ percentPeak $\times\left|p_{i, j}\right|$, then the walk is complete in that direction and the previous point, $\left|p_{i-n-1, j-m-1}\right|$, is the final point included in the interaction site prediction. The predicted interaction site on each protein is given by the minimum and maximum values found for $\mathrm{i}$ and $\mathrm{j}$ found during the walk algorithm. In the case of the PIPE experiments run for this thesis the cutoff percentage for the PIPE sites walk algorithm was $50 \%$. In order to filter out possible false positives caused by sharp peaks that occur from commonly occurring sequences, a minimum of number of walk steps in each direction is set. For all experiments run, the minimum number of walk steps was set to one.

In Figure 2.4 the interaction site would be the red/white area for a percentPeak value of $50 \%$. Figure 2.5 shows the same peaks in two dimensions with contour 
lines. The contour view shows that the peak in the upper right hand corner is less likely of being an interaction site as it has a very narrow base around its peak, compared with the lower left peak, which has a very wide base.

When reporting the interaction sites, PIPE-Sites will report at most the three PIPE sites from the three highest peaks that meet the criteria discussed. These three peaks are reported in order of peak height, the first site reported has the highest peak, the second is the second highest peak and so on. If there are two peaks with overlapping areas, both sites will be reported. An example of this can be seen in Figures 2.6 and 2.7. In this case the three sites that would be reported would come from the base areas of the three peaks in the lower left hand area of Figure 2.7, since these are the highest peaks. Each of the sites has some overlapping portion of the interaction site.

The interaction sites predicted by PIPE-Sites do not necessarily correspond to the interface between two proteins when they interact. However, PIPE-Sites does identify the sites that are important to the interaction in question whether they are on the surface, at the interface or buried within the protein.

\subsection{Limitations of PIPE}

While MP-PIPE represents a significant step forward towards computing a complete human interactome, there remain a number of limitations. In order to operate at a reasonable precision rate, we have tuned our decision thresholds to be extremely conservative, resulting in a limited sensitivity of $23 \%$, which is relatively low.

Where MP-PIPE has advantage over structure-based methods is in coverage: MP-PIPE requires only sequence as input and is therefore applicable to all protein 


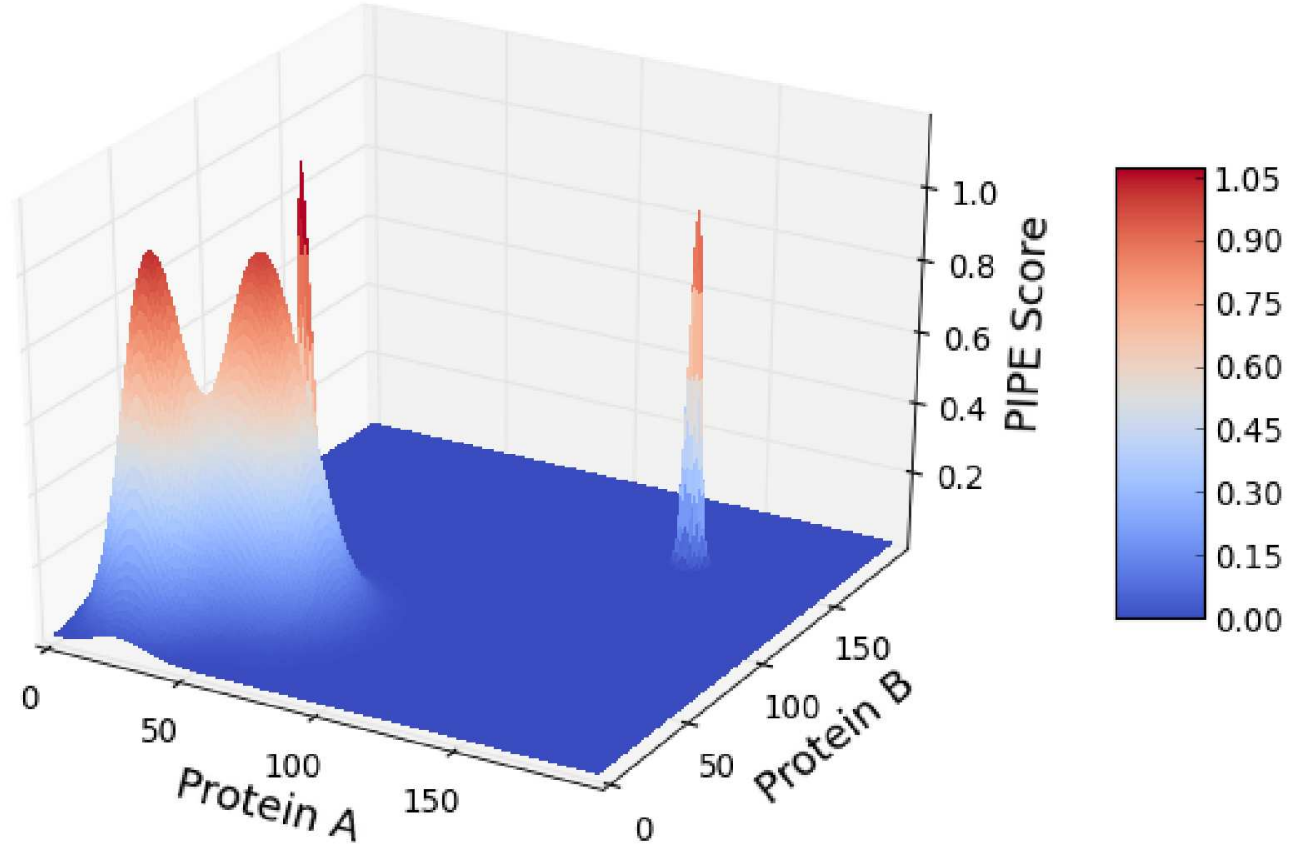

Figure 2.6: PIPE Score Surface Plot Showing More Than Three Peaks 


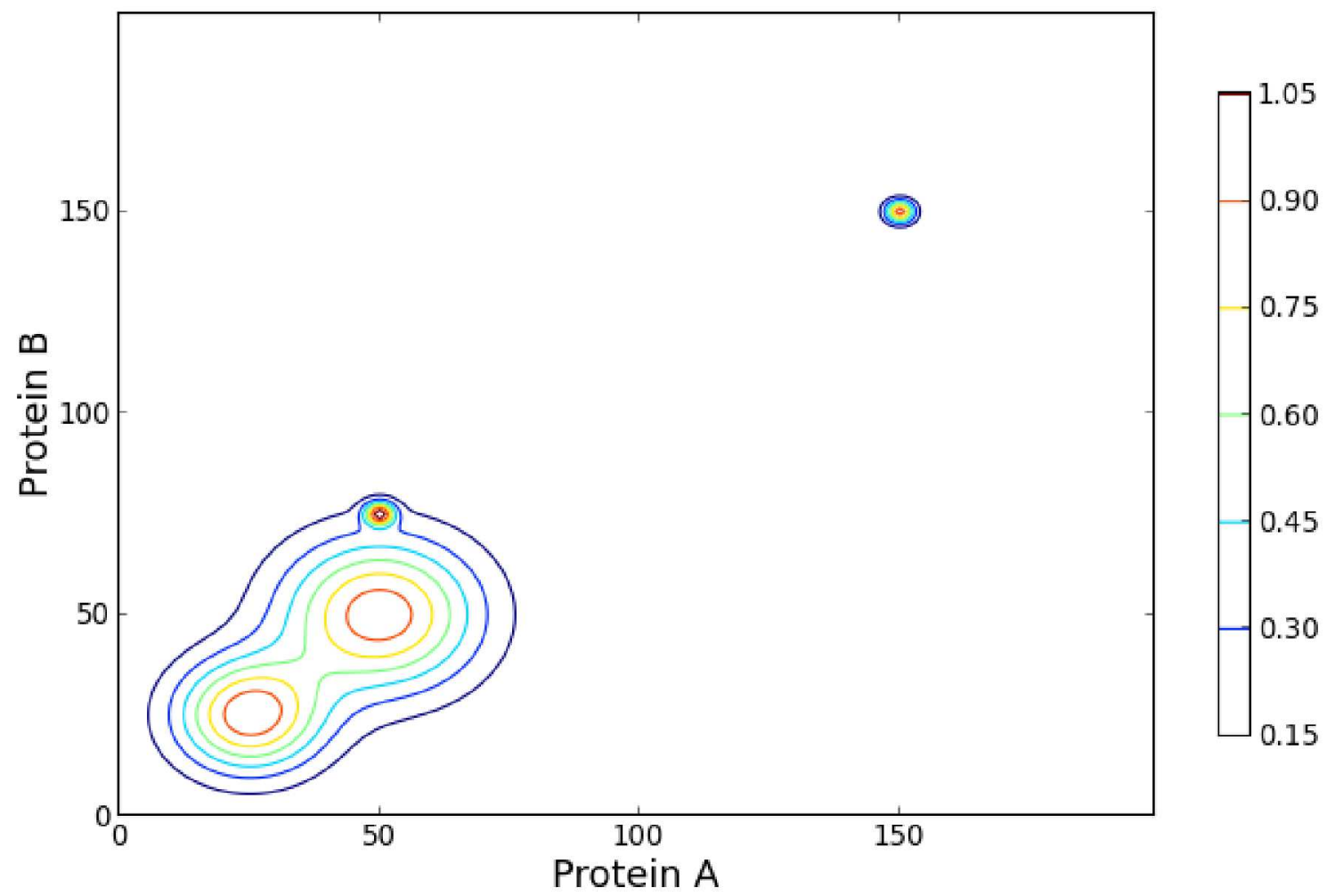

Figure 2.7: PIPE Score Contour Plot Showing More Than Three Peaks 
pairs. However, PIPE does not capitalize on the wealth of information available in the form of 3D protein structure data. At present, this represents only a small fraction of protein pairs, however, this proportion is expected to grow with ongoing large-scale protein structure determination initiatives.

As with all computational methods, another potential limitation in prediction accuracy is the quality of input data used to train MP-PIPE. As more experimental data of higher quality becomes available, we expect MP-PIPE to also become more accurate.

Lastly, further improvements to speed and parallelization 


\section{Quanitfying Performance of sequence-based PPI Prediction on SNPs}

To quantify the ability of sequence-based PPI prediction methods to detect the impact of SNPs on the interactome, the SKEMPI database, described in [7], was used. This database catalogs a series of point mutations on proteins and the corresponding effect on protein-protein binding affinity. The following sections describe the data, the methods, and the results related to addressing the first research question posed in Section 1.2.

\subsection{Data Source: SKEMPI database}

The SKEMPI database is a curated list of protein sequence mutations caused by SNPs in a number of organisms, including humans. The SNPs documented in the SKEMPI database do not necessarily occur in nature, as some of the data comes from experiments where mutations are made to DNA sequences in the lab with the goal of causing a change in PPIs [7].

Each mutation is listed as a reference to a wild-type Protein Data Bank (PDB) entry and has associated experimentally measured kinetic properties such as binding free energy, entropy, enthalpy and dissociation-rate constants.

PDB files describe the three dimensional structure of a protein and protein complexes, which are made up of multiple proteins. In the case of a complex of two or more interacting proteins, each of the individual proteins is labelled as a chain designated by a letter [4]. The data listed for each mutation includes only kinetic properties that were measured. Each mutation does not always have the same kinetic properties recorded. For both the mutant and wild type protein, it is 
important to note that the binding free energy, $\Delta \Delta G$, is recorded for all mutations [7]. The binding free energy is a measure of the strength of interaction between two proteins and a sufficient change indicates a loss or gain of interaction [3].

The SKEMPI database contains 3,047 $\Delta \Delta G$ measurements for 2,792 unique point mutations or sets of mutations. Of these mutations, there are 2,506 unique single amino acid substitutions applied to 158 different PDB protein-protein com-

plexes [7]. Each PDB chain's sequence for the mutated proteins were run through PIPE and the results analyzed. In total, including all chains of each PDB entry, the mutated chain sequences and the wild type sequences, there were 2,827 sequences and 65,300,873 test pairs that required the PIPE algorithm be run on them.

\subsection{Methods}

In order to use the SKEMPI data as an input for PIPE and sig_prod, some conversions were necessary to transform the data into the proper input format. Once converted, performance of both methods was evaluated.

\subsubsection{Data Conversion to PIPE Input}

There are a number of input parameters that needed to be computed from the SKEMPI proteins in order to run PIPE on the desired test interaction pairs from the SKEMPI database.

\section{Gentab: Generating the Protein Window Database}

Each PIPE run begins by precomputing the PIPE database of protein fragments used to generate the neighbor list $R$ via lookup instead of string-compare search, see Section 2.5.1 for the details. This database was generated using the parallel 
program GenTab on Carleton University's CGM Cluster. Algorithm 1 summarizes the Gentab program used to generate the PIPE database files.

The only required input for Gentab is the protein sequence file, which is a tab delimited file consisting of protein names and sequences for all proteins to be queried by the PIPE algorithm. The pseudo code in Algorithm 1 begins by loading parameters into arrays from the protein sequence file. Each array is indexed synchronously so the first sequence's corresponding length and name are the first entries in proteinLengths and proteinNames respectively. The numberOfProteins parameters is the number of proteins in the given sequence file.

Once all the parameters are loaded into their respective arrays, the sequences need to be converted from their character designations for amino acids to smaller binary number values of only 5 bits, which greatly decreases memory requirements.

After the conversion to a binary number protein sequence is complete, the parallel portion of the code begins. Each thread accesses a protein sequence from sequenceArray to operate on based on the processor rank assigned at the start of the MPI program. Each processor will then have an equal number of proteins to perform the sequence similarity search on. For each protein, the process compares each window of size $\mathrm{W}$ to all other windows of all other proteins, searching for similar sequences. The results are stored in a matrix, sequenceSimilarity Array, for each protein.

Once all comparisons are made, the resulting matrix results are output to the file. For this step only the proteins that have at least one similar window are recorded in the database file. Each protein has its own file recording the neighbour set, $R$, discussed in section 2.5.1. 


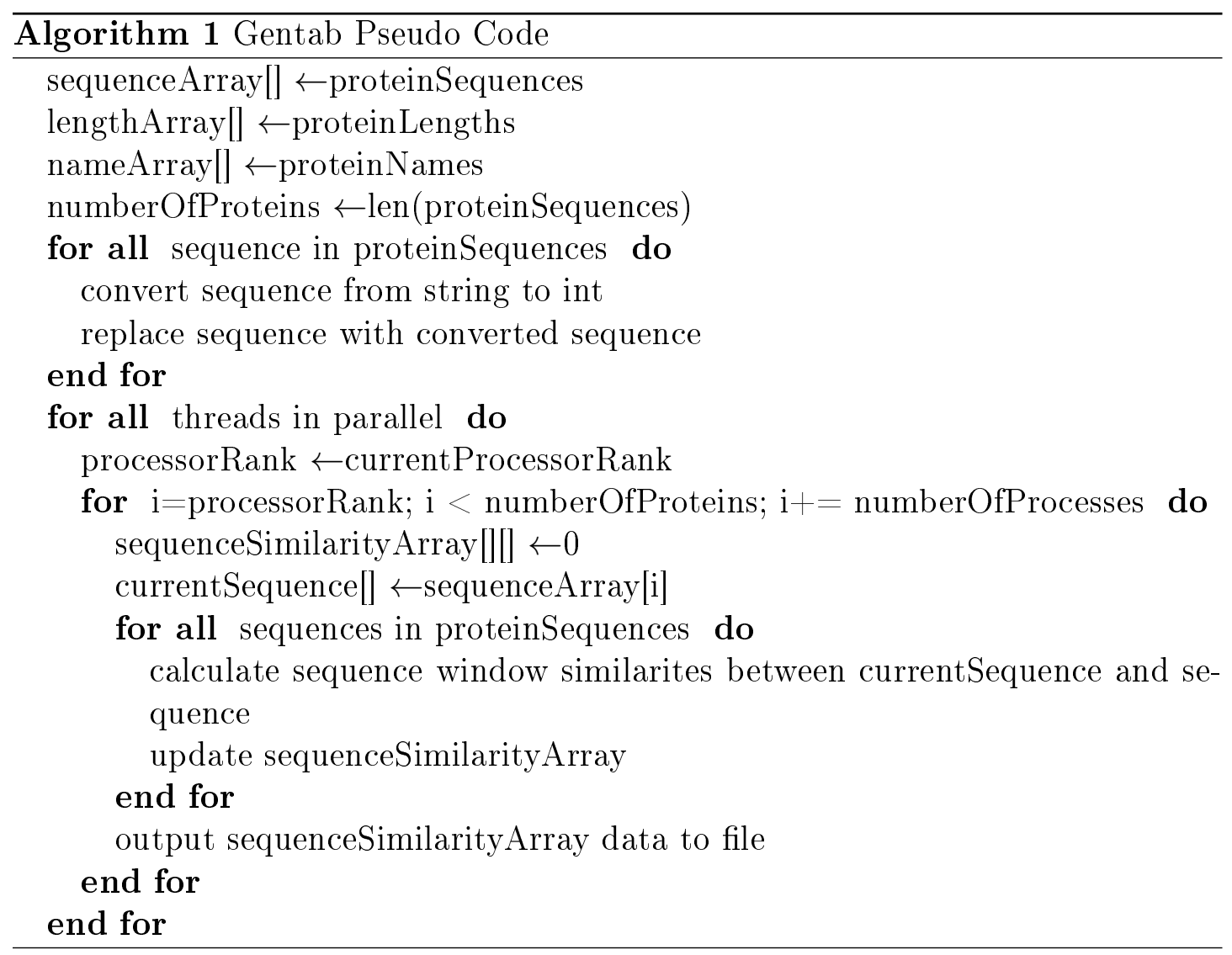




\begin{tabular}{|c|c|}
\hline Amino Acid Character & Amino Acid Binary Number \\
\hline \hline A & 00000 \\
\hline R & 00001 \\
\hline N & 00010 \\
\hline D & 00011 \\
\hline C & 00100 \\
\hline Q & 00101 \\
\hline E & 00110 \\
\hline G & 00111 \\
\hline H & 01000 \\
\hline I & 01001 \\
\hline L & 01010 \\
\hline K & 01011 \\
\hline M & 01100 \\
\hline F & 01101 \\
\hline P & 01110 \\
\hline S & 01111 \\
\hline T & 10000 \\
\hline W & 10001 \\
\hline Y & 10010 \\
\hline B & 10011 \\
\hline Z & 10100 \\
\hline X & 10101 \\
\hline U & 10110 \\
\hline & 10111 \\
\hline
\end{tabular}

Table 3.1: Amino Acid Conversion Table 


\subsubsection{Creating Mutated Protein Sequences from PDB Files}

It was challenging to identify a consensus amino acid sequence for each protein chain from the PDB file itself since the information contained therein relates to the 3D structure of the protein and not a linear sequence of amino acids. To overcome this challenge a mapping file was acquired to map the proper sequence of amino acids for each of the PDB chain references in the SKEMPI database. This mapping file is available from [27] and can directly map the linear amino acid sequence of PDB chains from their respective PDB entries. These sequences were then used in the PIPE algorithm.

Not all of the PDB proteins in the mapping file had a linear sequence and as a result, 2,376 of the 2,506 mutations mapped to PDB amino acid sequences. Of these 2,376 mutations, 1,349 occur in PDBs from the human proteome, comprising over $50 \%$ of the mutations that mapped to PDB sequences. Including all mutated and wild-type chains, there were 2,827 protein sequences requiring a one-to-all PIPE run.

Once the sequences for all the PDB chains were acquired, the PIPE algorithm was run between each chain and all other chains as well as to all Uniprot proteins in the PIPE database. This was effectively a one-to-all comparison for each PDB chain. Figure 3.1 shows a PDB entry, 1FCC, with two chains, A and B. The mutated sequence of chain A is labelled, for PIPE, as:

1FCC_A_ $<$ wild_type_amino_acid $><$ location $><$ mutant_amino_acid $>$

The wild type chain A is labelled similarly but without the mutation information. Both of these sequences have PIPE one-to-all runs, with PIPE being run between each sequence and all other PDB sequences as well as the Uniprot proteins already used in PIPE. On top of the mutated and wild type sequences for the mutated 


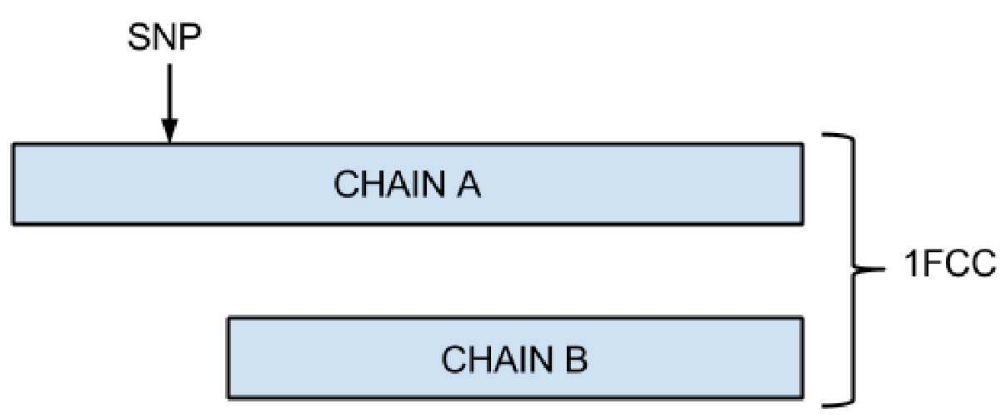

Figure 3.1: Chains from a PDB Entry

chain, the non-mutated chain, B in this case, also has a PIPE one-to-all run performed. This wild type chain was added in order to validate PIPE's sensitivity in examining the interaction between the two chains from the PDB entry.

\subsubsection{Generating Input Pairs for Individual PIPE SNP Runs}

To generate the files needed to run PIPE, scripts were written in Python. Python was selected as a language because of its simple and fast string manipulation syntax. There are only three files needed to begin the process of creating all inputs for the PIPE runs. They are:

1. Protein sequence file for all mutated proteins - contains only the mutated sequences for the SNPs, 2,827 in total

2. Protein sequence file for all wild type proteins - contains the wild type sequences, 20,273 in total

3. Original interaction graph file - file containing all known protein interactions These files must all be tab delimited.

The first script used generates the one-to-all input pairs for each SNP and each unmutated wild-type protein. 
This script has the following usage:

python create_sets_against_all_input.py <input1> <input2> <input3>

Where input1 is the sequence file only containing the sequences for mutated proteins, input2 is the wild type sequence file containing only the sequences for the non-mutated proteins and input 3 is the destination folder for all the input pair files. Further details on generating the input parameters and other information can be found in Appendix A.

\subsubsection{Running PIPE on the M9000 Enterprise Server}

PIPE is very portable to large scale parallel systems. In total, for the new 2,827 protein sequences, there are $65,300,873$ pairs to be run. Sequentially if each pair takes $780 \mu$ s to evaluate, as discussed in Section 3.2.5, this would require over 15 days of sequential CPU time. Obviously a large scale parallel system was required to complete the necessary PIPE runs for each SNP.

Compute Canada is an organization that manages High Performance Computing (HPC) resources in Canada. They provide these resources to research groups based on need, which is described through an application process. The High Performance Computer Visualization Laboratory (HPCVL) is one of the facilities managed by Compute Canada and the PIPE project was assigned an M9000 Enterprise server for use in this specific project from the HPCVL. The HPCVL uses the software GridEngine to manage resource allocation, however there were no issues getting CPU time for this project as an entire M9000 Enterprise server was dedicated for use by the PIPE project.

The M9000 is made up of 64 quad-core SUNSparc64 VII 2.53GHz processors. Each core itself is capable of executing 2 hardware threads through chip 
multi-threading. These components make the M9000 capable of running 512 simultaneous threads. Each core has $8 \mathrm{~GB}$ of RAM for a total of $2 \mathrm{~TB}$ of RAM. The architecture is shown in Figure 3.2, each hardware thread is labelled with an $a$ and $b$ respectively. Since the SUNSparc VII is a different architecture to the standard $\mathrm{x} 86$-based multicore processors, a different compiler was used. The compile command used was:

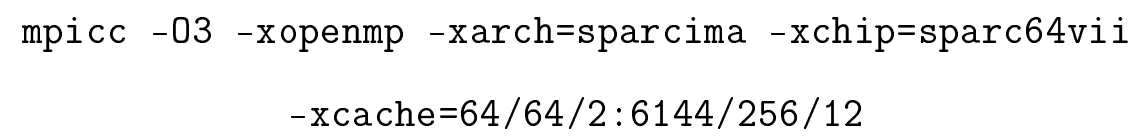

The amount of RAM available makes the M9000 particularly well suited to high memory applications, like that of running a high number of PIPE worker threads. Due to the highly variable length of proteins in the human proteome, there are a few proteins that are significantly longer than the others. Since PIPE currently allocates memory based on the maximum length of all proteins, this has led to memory issues in the past when PIPE has been run on clusters that have less memory per node available. In these cases the longer proteins in the interactome were run separately in order to speed up execution.

It is important to note that the M9000 uses the Big Endian file convention when interpreting binary files. This is important because the PIPE database is generate using the Little Endian byte reading convention and had to be converted prior to using it on the M9000.

On the M9000 the PIPE program is called as follows:

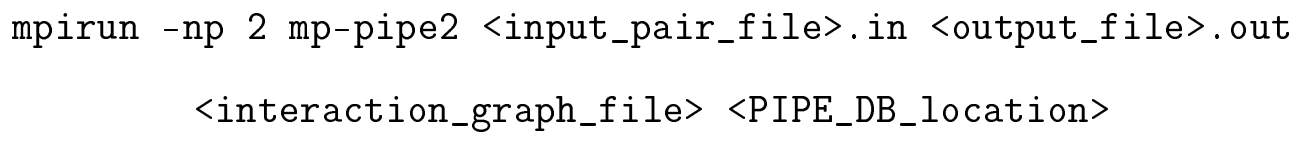

As described previously, only a single master and one slave is used since the 


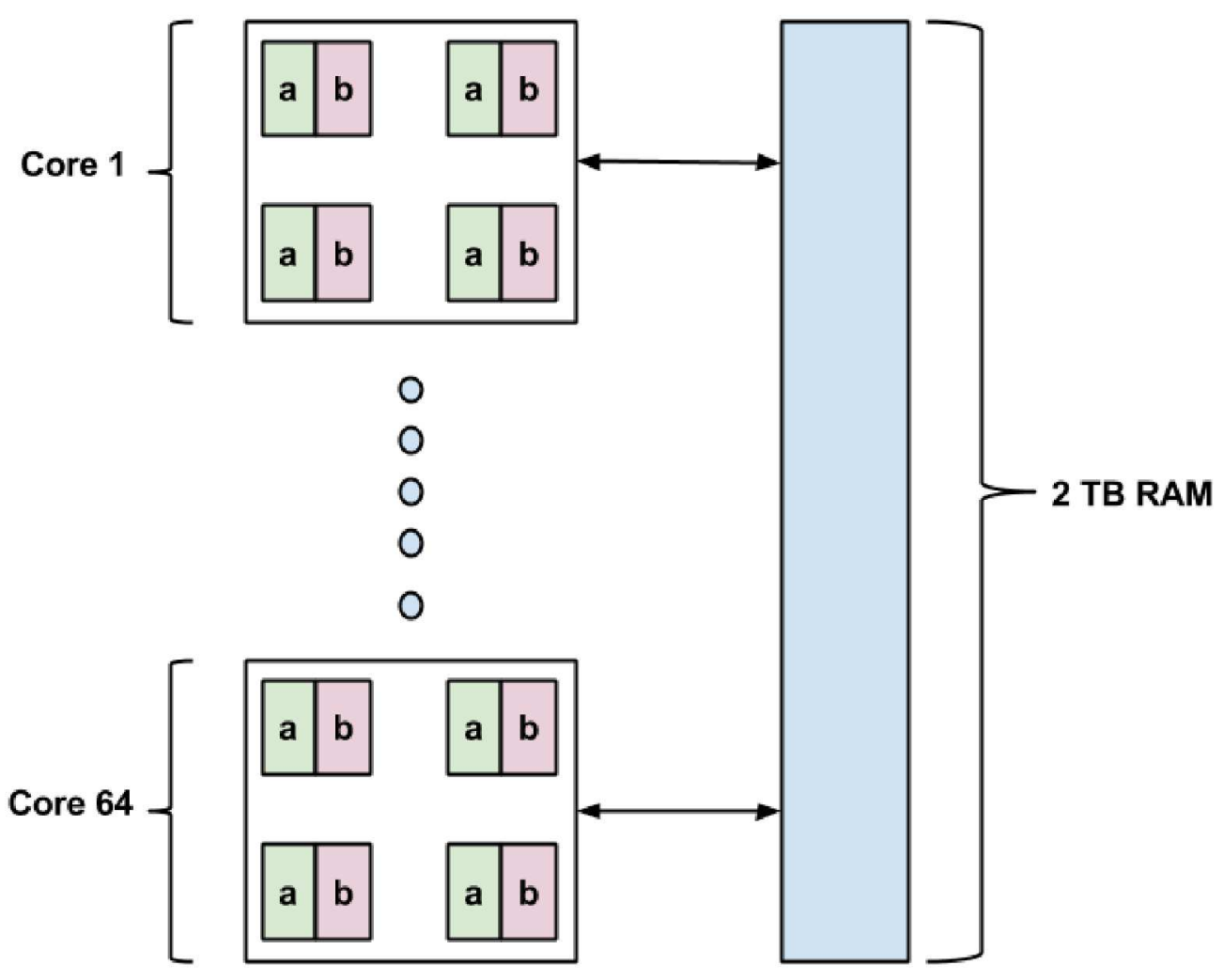

Figure 3.2: M9000 Enterprise Server Hardware Architecture 


\begin{tabular}{|c|c|c|}
\hline Threads & Run Time(s) & Speedup \\
\hline \hline 64 & 84065 & 82 \\
\hline 128 & 54210 & 127 \\
\hline 256 & 51222 & 135 \\
\hline 320 & 50959 & 136 \\
\hline
\end{tabular}

Table 3.2: M9000 Run Time Information

M9000 has such a large amount of memory, however, the single slave has a large number of worker threads.

\subsubsection{Run Time Information for M9000 PIPE Runs}

The M9000 performance was benchmarked using the SKEMPI PIPE data since it contained the largest number of pairs for which PIPE needed to be run.

As discussed in Section 3.1, the sequences from the PDB chain entries in the SKEMPI database had the PIPE algorithm run on them. In total there were 2,827 additional sequences added to the sequence library. For all the one-to-all runs for each of the new sequences there were $65,300,873$ test pairs on which PIPE needed to be run. These pairs were run on the M9000 using the full PIPE interaction graph. The PIPE algorithm used 320 threads and completed all predictions in 14.15 hours, which equates to approximately $780 \mu \mathrm{s}$ per test pair.

Table 3.2 shows a summary of the run time information in seconds. As can be seen there is significant speedup between 64 and 128 threads, however it plateaus for more threads, most likely due to the time taken due to allocating memory for the output files. The speedup was calculated using a sequential runtime estimate using the per pair calculation of $106 \mathrm{~ms}$ multiplied by the 65 million pairs to be computed which results in a sequential time of 6937564 seconds. This estimate was calculated using the runtime from the 128 thread PIPE run. 


\subsubsection{Alternative PPI Prediction Methods}

In [15] there are four sequence-based PPI prediction methods compared and measured against one another, included in these methods was PIPE. In order to compare PIPE's ability to detect interactome changes from SNPs against a competing method, these other PPI prediction methods were researched for availability of their source code. The only other method available was the method labelled M1 in [15] and is a method from [8]. This method uses signature products based on the amino acid sequence to convert each protein into a uniform length vector. A Support Vector Machine (SVM) is then trained on these vectors and used to make predictions. This method, called sig_prod, was applied to a subset of the SKEMPI SNPs and compared with PIPE's performance on the same subset of SNPs.

\subsection{Results for SKEMPI Data}

The initial data taken from SKEMPI and run through PIPE was discussed in Section 3.1. This set of SNPs provided the basis for the evaluation of PIPE's performance in predicting SNP effects on the interactome.

As discussed in Section 2.5, PIPE provides a score between 0 and 1 as a measure of the likelihood that two proteins interact. The metric to evaluate PIPE's performance here was the difference between mutant and wild type PIPE score. This score was calculated as score $_{\text {mutant }}-$ score $_{\text {wild }}$ and was then compared with the corresponding protein affinity change in the SKEMPI database.

All analysis was done using a PIPE threshold of 0.90849870 , which corresponds to a specificity of 0.9995 . The PIPE threshold was calculated using the leave-oneout cross-validation methodology detailed in [1], where an interaction is removed 
from the interaction graph and then PIPE is run on the removed pair to validate whether PIPE will predict this interaction if removed. This decision threshold is used to determine the cutoff for predicting an interaction between two proteins.

\subsubsection{PIPE Score Change for SKEMPI SNPs}

Initially the overall mean score change and standard deviation were calculated. This result was calculated for all interactions, not just interactions above the PIPE threshold. The mean change was $7.2267 e-4$ with a standard deviation of $1.3994 e-2$. The mean change in this case indicates that there is usually an increase in PIPE score, however since the standard deviation is high relative to the mean, there is high variability in this PIPE score change. Regardless, the magnitude of change is very small.

Since the mean change includes both positive and negative changes, some information is lost by simply looking at this one measure of change in PIPE score. The mean of the absolute value of all PIPE score changes was also calculated in order to better assess the magnitude of changes caused by a change in PIPE score.

The mean for the absolute value of the PIPE score change was found to be $2.9076 e-3$ with a standard deviation of $1.3707 e-2$. Once again the magnitude of these changes is small, which is as expected when considering the sliding-window algorithm used by PIPE.

Figure 3.3 shows a histogram of the PIPE score changes. As can be seen in the histogram, most mutations caused very small changes in PIPE score.

The interactions that were not predicted to interact by PIPE were also examined for their score change. The mean score change for these interactions was $4.73 e-04$ with a standard deviation of $8.80 e-0.03$. The absolute change had a mean of $2.30 e-03$ with a standard deviation of $8.56 e-03$. The standard 


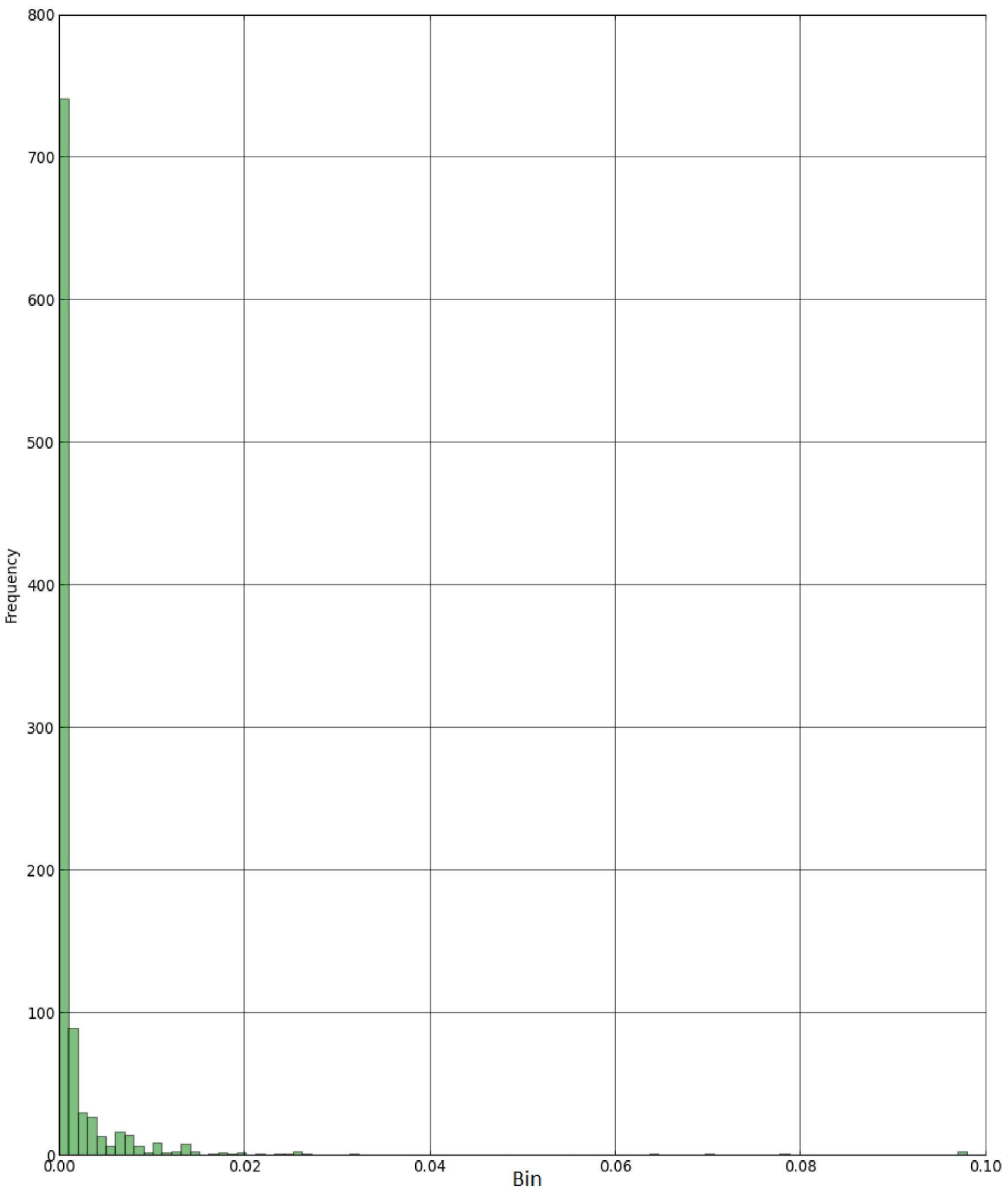

Figure 3.3: Histogram of Absolute PIPE Score Changes 
deviation is sufficiently large for both means that this difference from the PIPE predicted SKEMPI interactions is not significant. It is interesting to note that even though two proteins are not predicted to interact, they still exhibit a change in magnitude. This result may indicate that PIPE score could be used in some way to predict SNP-induced interactome changes regardless of whether the two proteins are predicted to interact or not.

\subsubsection{PDB and Affinity Analysis of SKEMPI Data using PIPE}

Each chain in a PDB entry is a part of a PDB protein complex. These PDB entries offered an opportunity to validate PIPE as a prediction method by seeing if PIPE will predict an interaction between the various chains of a PDB complex. With this in mind, each non-mutated chain pair from each PDB chain pair from the same complex had their PIPE scores compared to the PIPE threshold. In total PIPE predicted 407 of 2,183 chain pairs to interact, corresponding to a sensitivity of $18 \%$, which aligns with published performance standards related to PIPE $[15,6,1]$. The decision threshold for the PIPE algorithm has been tuned to be very conservative, sacrificing sensitivity to achieve a very low false positive rate. This decision is critical for applying PIPE to all protein pairs, considering the relative sparcity of the interactome.

While PIPE score changes are quantitatively small, it was hoped that they can still provide guidance in some way as to how a mutation will affect an individidual's interactome. To this end, comparing the PIPE score between mutated and wild type chains with experimentally observed protein affinity change offers another chance to validate PIPE as a method which can be used to predict interactome changes from SNPs. The PIPE score change and protein affinity change comparison has a total of 1,783 data points, made up of the mutations from 


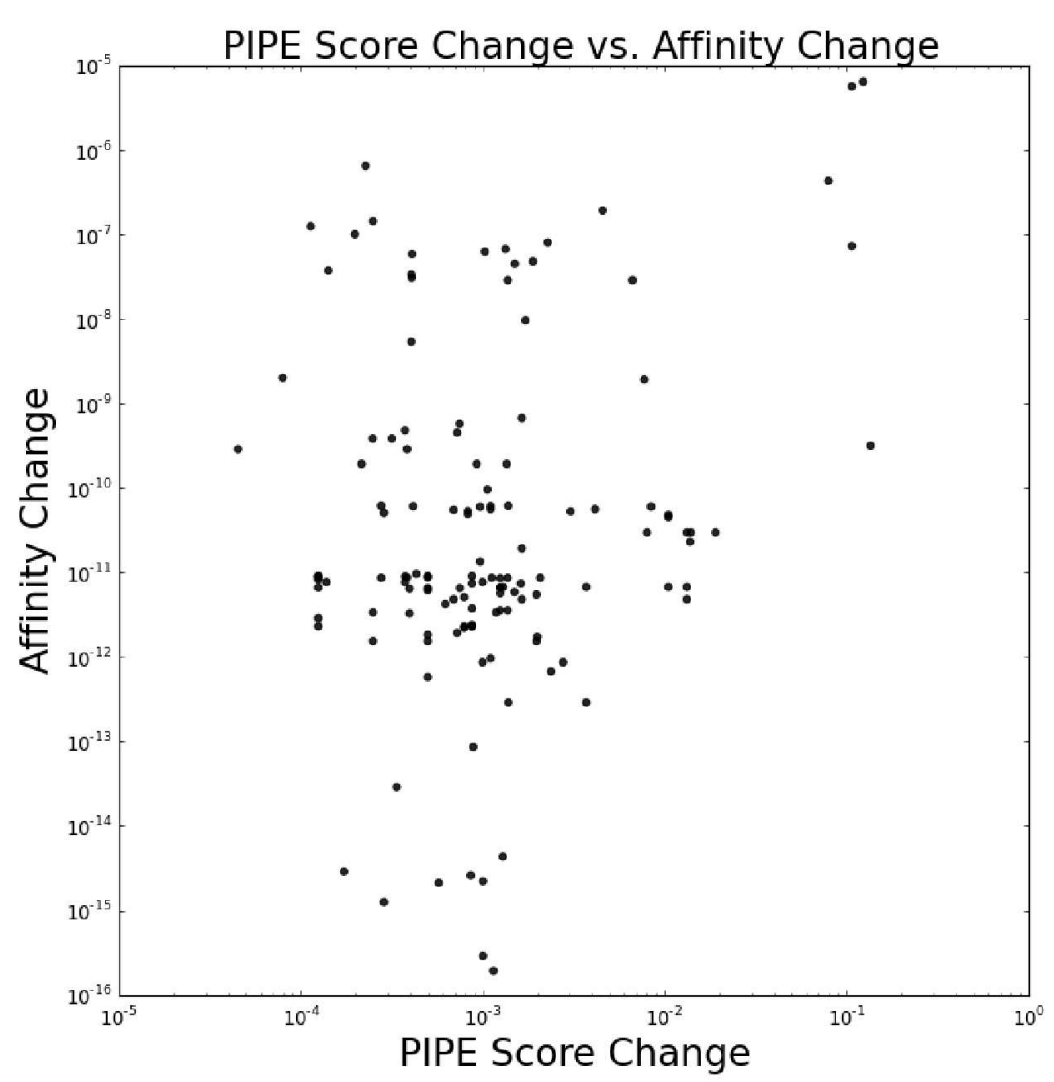

Figure 3.4: PIPE Score Change vs. Protein Affinity Change for SKEMPI SNPs

SKEMPI. There is not a strong correlation, however some pairs do appear to correlate higher changes in PIPE score with high changes in affinity. Figure 3.4 plots the protein affinity change along a log-scale y-axis and the PIPE score change along a log-scale x-axis. As can be seen there is virtually no correlation between protein affinity change and PIPE score change. With a log-curve fit to the data shown in Figure 3.4, the resulting correlation was 5.36e - 04, which validates the obvious lack of correlation. 


\subsubsection{Alternative Sequence-Based PPI Method Results using SKEMPI Data}

As discussed previously in Section 3.2.6, one alternative method, sig_prod, was used to make predictions on the SKEMPI dataset for comparison with PIPE's performance. Since this method is based on a Support Vector Machine, positive and negative interactions are needed to train the classifier to recognize characteristics of each. The PIPE interaction graph was used as the positive set.

The negative set was created using the methodology described in [28]. Using this methodology, interactions were generated uniformly at random. Ideally each protein from the positive interaction set would have the same number of occurrences in the negative interaction set, however sig_prod can only handle approximately 150,000 training pairs, so this constraint was relaxed. Instead of proportional occurrences of each protein in the positive and negative sets, each protein in the positive set of interactions occurs at least once in the negative set, which is an alternative method for negative set generation described [28].

The same set of data from SKEMPI, described in 3.1, was used to generate Figure 3.5. Similar to Figure 3.4, a log-scale was used on both axes. Again, this sequence-based method does not appear to have any correlation between the SNP-induced affinity changes from SKEMPI and the score changes in sig_prod.

This result is hardly surprising. The nature of sequence-based methods minimizes the impact a single amino acid change can have on the interactome. 


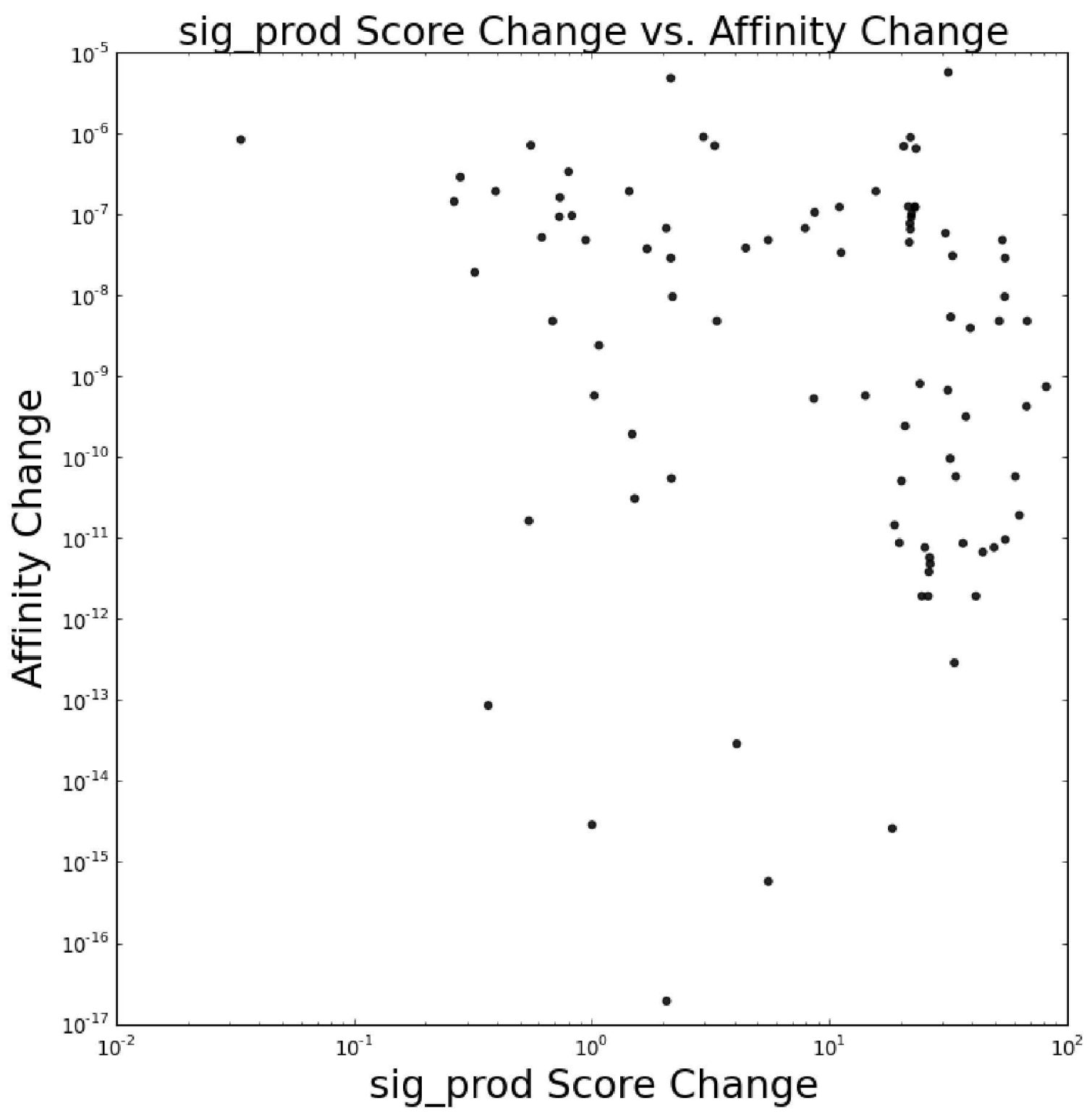

Figure 3.5: sig_prod Score Change vs. Protein Affinity Change for SKEMPI SNPs 


\subsection{Conclusion}

From the results shown in this Section, sequence-based PPI methods are not sensitive to SNP-induced interactome changes, based on the protein-affinity changes documented in the SKEMPI database. 


\section{Using PIPE-Sites to Identify Interactome Changes Caused by Disease-associated SNPs}

As expected, the PIPE scores from Section 3 did not exhibit any large changes in magnitude due to non-synonymous SNPs. This is due to the nature of the PIPE algorithm described in Section 2.5.1, whose sliding window approach will tend to reduce the effect a single amino acid substitution can make to the overall protein and interactome.

We next examined the location of the SNP, relative to the predicted location of the PPI interface, in conjunction with PIPE score change to see if a change or pattern could be extracted by incorporating this feature. The PIPE-Sites feature of PIPE was also used to identify subsets of SNPs which are enriched at predicted interaction sites. It is hoped that interactions which show statistically significant enrichment of SNPs at a PIPE site are more likely affected by those SNPs.

As discussed in Section 2.5.2 the PIPE interaction site prediction provides the top three most probable interaction sites, if they are available based on the constraints stated in that Section.

\subsection{Data Source: SNPdbe}

One of first challenges in evaluating PIPE-Sites in conjunction with PIPE score change was to find a dataset of known SNPs that would theoretically cause drastic changes to the human interactome. There are a number of organizations and projects that annotate and verify SNPs. Fortunately the SNPdbe project has amalgamated a number of SNP data sources into a single MySQL database which is publicly available. This database provides a collection of SNPs with annotated 
information such as predicted functional effect, disease association, source and experimental information. For the purposes of this project and due to the availability of data, the mutations from SNPdbe were only mined for those associated with disease.

The SNPs in SNPdbe were collected from four sources, Uniprot, 1000 Genomes (1KG), dbSNP and the Protein Mutant Database (PMD). In total there are 1,362,793 unique SNPs in 158,004 proteins from 2,684 organisms [4].

This research focused on SNPs in the human genome that directly mapped to the input format for proteins in PIPE, which uses Uniprot as the naming convention for protein information. Disease causing SNPs in particular were targeted with the expectation that these SNPs may cause disease through disruption of PPIs, and would therefore lead to more drastic changes to the interactome. In total 25,804 disease-causing SNPs were successfully harvested from the SNPdbe MySQL database.

Although we cannot be certain that these SNPs cause their associated disease through disruption of PPIs, we hypothesize a subset of these SNPs do this, and we aim to develop an approach to mine the interactome to identify likely candidates.

\subsubsection{SNPdbe PIPE Run Data}

Since each PIPE run requires a significant amount of run-time and resources, a subset of these disease-causing SNPs was selected to benchmark PIPE's performance on predicting the affect of SNPs in conjunction with PIPE-Sites. This subset is summarized in Table 4.1. In consultation with biologist collaborators, these diseases were targeted as it was felt that they potentially have more effect on protein interactions rather than protein function compared with other diseases in the disease list. Another factor in the selection of diseases used for the initial 


\begin{tabular}{|c|c|c|c|}
\hline Disease & $\begin{array}{c}\text { Number of } \\
\text { SNPs }\end{array}$ & $\begin{array}{c}\text { Number of } \\
\text { Proteins }\end{array}$ & $\begin{array}{c}\text { Mean SNPs } \\
\text { Per Protein }\end{array}$ \\
\hline \hline $\begin{array}{c}\text { colorectal adenocarcinoma } \\
\text { hereditary non-polyposis colorectal } \\
\text { ăcancerătype 2 }\end{array}$ & 84 & 55 & 1.52 \\
\hline familial hypercholesterolemia & 90 & 1 & 90 \\
\hline breastăcancer & 845 & 566 & 1.49 \\
\hline $\begin{array}{c}\text { familial hypertrophic } \\
\text { cardiomyopathy } \\
\text { type 1 }\end{array}$ & 167 & 1 & 167 \\
\hline Total & 1276 & 624 & 2.00 \\
\hline
\end{tabular}

Table 4.1: Disease SNPs from PIPE Benchmark Run

PIPE performance benchmark was the number of SNPs for each disease. Only diseases with 50 or more SNPs were considered.

This subset of SNPs was used only for the PIPE score change analysis in Section 4.3.5. All PIPE-Sites location analysis in Sections 4.3.1, 4.3.2 and 4.3.3 used all 25,804 SNPs harvested from SNPdbe.

\subsection{Methods for PIPE-Sites SNPdbe Data Analysis}

All 25,804 SNPs acquired from SNPdbe are associated with a congenital disease. Theoretically these SNPs have the greatest potential effect on protein function and protein interactions. Comparing the location of these harmful SNPs to that of PIPE sites for various interactions could provide insight into which SNPs affect protein interactions and which affect protein function in different ways. We seek to identify interactions with a statistically significant enrichment of SNPs within the predicted interaction interface, as we hypothesize that such interactions are more likely to be impacted by SNPs.

Various comparisons and statistics were used to analyze the location of all 25,804 SNPs and identify any statistically significant interactions that have a 
high probability of being affected by SNPs.

The PIPE score change was also analyzed for the 1,276 SNPs that are detailed in Section 4.1.1. These SNPs were run through PIPE and had their PIPE score change analyzed in conjunction with PIPE-Sites data. The details for the PIPE runs for this subset of SNPs can be found in Section 4.2.6.

\subsubsection{P Value Calculation}

In order to assess whether the proportion of SNPs that occurred in PIPE sites was significant, a p-value was required. Since the population size was large, a binomial distribution was used.

The equation used to calculate the Probability Density Function(PDF) value was:

$$
f(x=k)=\left(\begin{array}{l}
n \\
k
\end{array}\right) p^{k}(1-p)^{n-k}
$$

Each parameter in Equation 4.1 has a specific value that varied with each different type of analysis done on the data.

The " $n$ " parameter, which is the population size, was the number of total SNPs for that particular calculation.

The " $\mathrm{k}$ " parameter, which is the number of positive observations, was always assigned to be the number of SNPs that fell in a PIPE site.

The "p" parameter, which is the probability of observing an instance of " $\mathrm{k}$ ", was the probability of a SNP falling in a PIPE site. This value was represented by the proportion of the protein covered by the PIPE site.

The equation used to calculate the Cumulative Distribution Function(CDF) value was:

$$
P(X \leq x)=\sum_{i=0}^{x}\left(\begin{array}{c}
n \\
i
\end{array}\right) p^{i}(1-p)^{n-i}
$$


Where $x$ is the number of SNPs that occur in the PIPE site. By subtracting the value generated from equation 4.2 from 1 , the resulting value represents the probability that the results ( i.e. the observed level of enrichment or higher) were generate by chance, also described as $P(X>x)$. For the most part, the analysis was done at a significance level of 0.05 , meaning there is a $5 \%$ chance the observations were from a random population and there is actually no correlation.

All of the analysis for the interactions discussed was only done for interactions that were predicted to be positive by PIPE with a specificity of 0.99945003 and a sensitivity of 0.23766370 . The PIPE score threshold used to achieve these values was 0.90849870 .

\subsubsection{Per-Site Analysis of SNP Locations}

There were a number of ways PIPE-Sites could be used to analyze enrichment of SNPs. Since each site could be a different interaction interface, each PIPE site within all proteins was examined individually for any enrichment of SNPs. Interactions which had statistically significant enrichment of SNPs at a PIPE site are likely affected in some way by the SNPs.

The initial analysis looked at each of the top three PIPE sites as a separate binomial event that was assigned a p-value based on enrichment of SNPs. This analysis was labelled as "per-site" analysis. In this case, each site is analyzed separately.

The value for $p$ in Equation 4.2 in this case is taken to be the proportion of the protein that is covered by the PIPE site in question. For a protein $x$ this can be calculated using the formula:

$$
p=\frac{\mid \text { site }_{i} \mid}{|x|}
$$



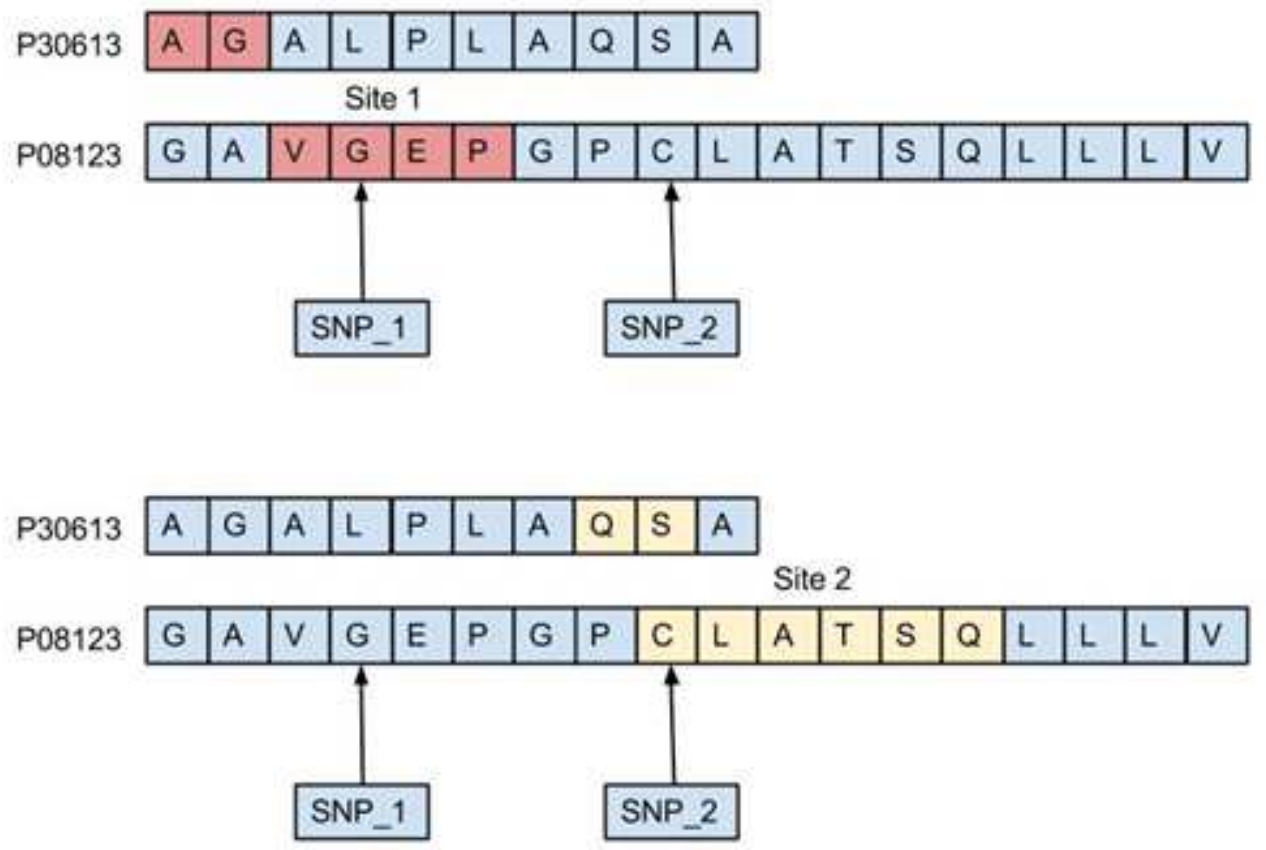

Figure 4.1: Per Site Interaction P-Value Analysis

Where $|x|$ is the length of protein $x$ in number of amino acids and $\mid$ site $_{i} \mid$ is the length of the interaction site $i$ in number of amino acids.

The number of positive occurrences, $k$ from Equation 4.2, is calculated as the total number of SNPs that fall into this single PIPE site, site ${ }_{i}$.

The population size, $n$ from Equation 4.2, is calculated as the number of SNPs from the SNPdbe database, that occur in the protein in question.

Figure 4.1 shows two PIPE sites from the same interactions. The first interaction in red covers $\frac{4}{18}$, which corresponds to $p$ from Equation 4.2. Correspondingly for Site 1 labelled in red, $n$ would be 2 and $k$ would be 1 . Site 2, highlighted in yellow, would have the same $n$ and $k$ values but would have a value of $\frac{6}{18}$, since the PIPE site covers more of the protein length. 


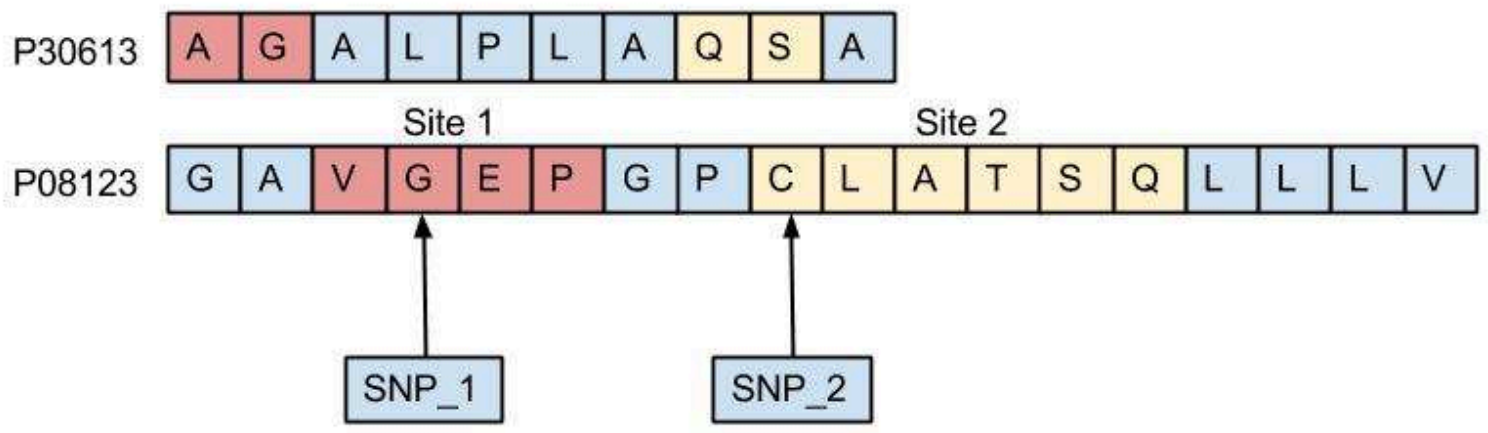

Figure 4.2: Per Protein Per Interaction Analysis

\subsubsection{Per-Protein and Per-Interaction, Site Analysis of SNP Locations}

The second form of analysis through which PIPE-sites was analyzed looked at each interaction for each protein in the dataset. Figure 4.2 shows an example of this analysis

In Figure 4.2 one interaction is between two proteins. In this case there are two interactions sites for P08123 and two SNPs. For this interaction, the coverage, $p$, is calculated as the proportion of the protein covered by both PIPE sites predicted for this interaction. Equation 4.4 shows the calculation for the coverage for all PIPE sites of an interaction, which is the length of all PIPE sites together divided by the length of the protein itself.

$$
p=\frac{\mid \text { site }_{1 x} \cup \text { site }_{2 x} \cup \text { site }_{3 x} \mid}{|x|}
$$

Once again, $k$ is the number of SNPs that occur in one of the PIPE sites for that interaction and $n$ is the total number of SNPs that occur in that protein. Each predicted interaction for P08123 was analyzed in this way and a p-value generated. 


\subsubsection{Per Disease Analysis}

The analysis carried out on each disease analyzed all the proteins which had SNPs associated with that disease then looked at all the interactions associated with the mutated proteins. For each of these interactions a p-value was calculated using the per site calculation from Equation 4.3 for the site coverage, but with only the first PIPE site, which is also the highest confidence prediction of an interaction site.

The $n$ value for the p-value calculation in Equation 4.2 was the number of SNPs that occur from the disease for the protein in question.

The $k$ value for the p-value calculation in Equation 4.2 was the number of SNPs that occur in the first PIPE site of the interaction in question.

In almost all cases, only one of the two interacting proteins had SNPs associated with a particular disease. In the rare case where both of the interacting proteins each had a SNP associated with the disease in question, the site coverage was calculated as shown in Equation 4.5.

$$
p=\frac{\mid \text { site }_{1 x} \cup \text { site }_{1 y} \mid}{|x|+|y|}
$$

In this case, the $n$ value for the p-value calculation in 4.2 was the number of SNPs that occur from the disease for both the proteins in question.

The $k$ value for the p-value calculation in 4.2 was the number of SNPs that occur in the both PIPE sites. Figure 4.3 shows an overview of an example process used to analyze the SNPs on a per disease basis for colorectal cancer.

In Figure 4.3 The SNPs for colorectal cancer and their associated proteins are shown. For all of the predicted interactions, one of which is shown between proteins A0AV96 and Q15757, a p-value is calculated using the first PIPE pre- 

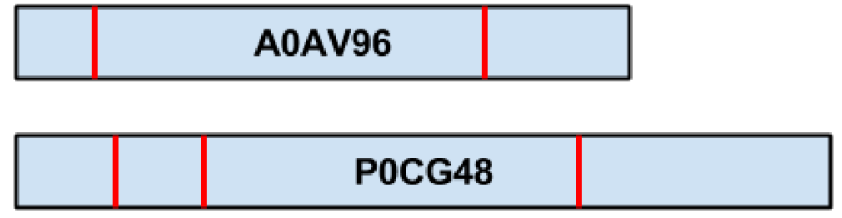

P62837
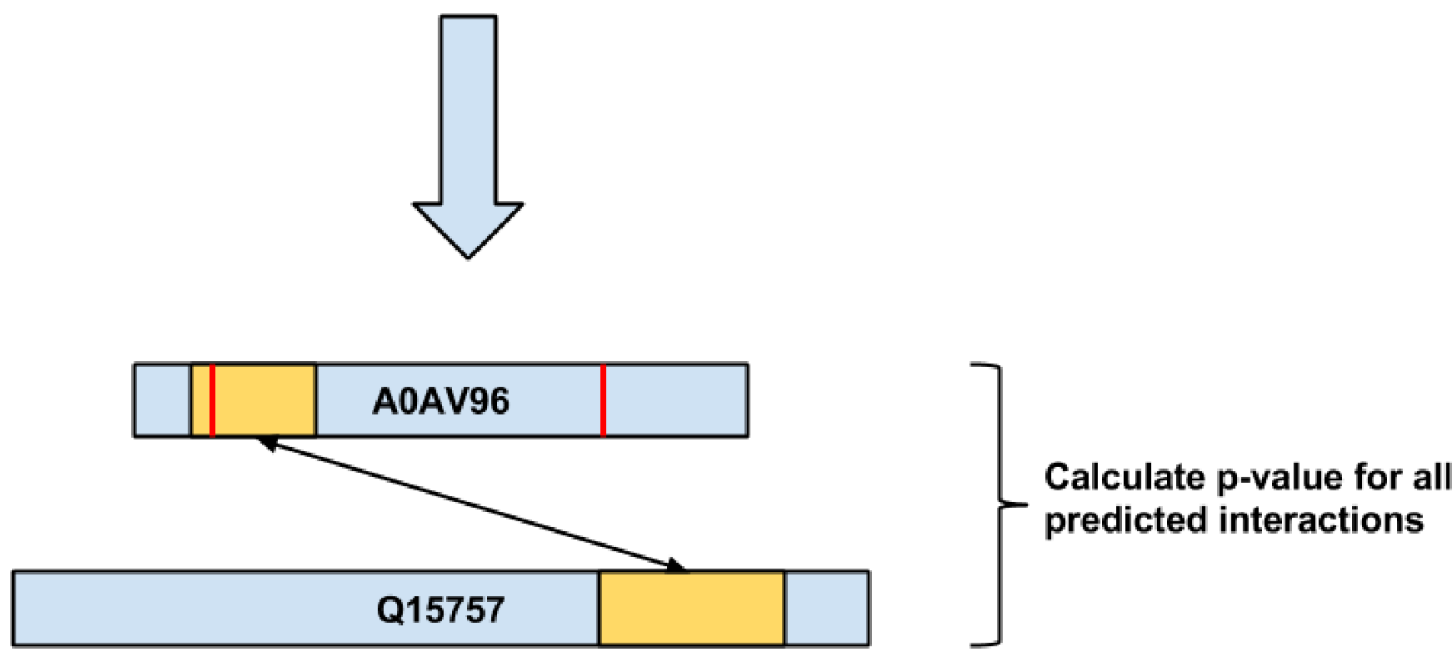

Figure 4.3: Per Disease P-Value Analysis 

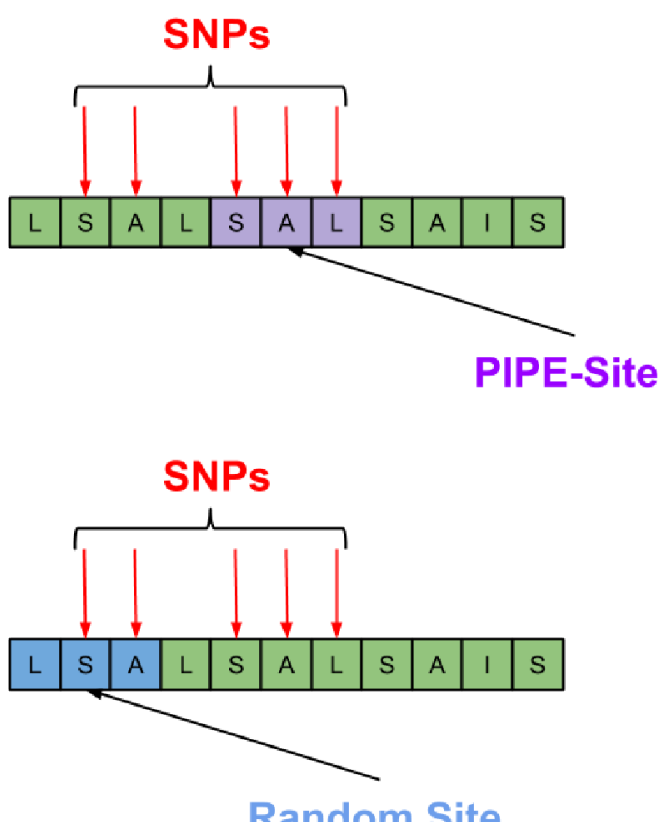

Figure 4.4: Randomly Generated Interaction Site

dicted interactions site. These p-values and the associated interactions were then recorded and analyzed.

In order to further validate the observed enrichment of SNPs at certain interaction sites, a random test was performed. In this test, for each interaction in each disease, a randomly generated interaction site of the same size as the PIPESite was generated and the p-value calculated as described earlier in this section. Figure 4.4 shows a protein with a PIPE-Site highlight in purple and SNPs shown in red. After analyzing the SNPs and PIPE-Site for statistically significant enrichment, a random site is generated and again statistically significant enrichment of SNPs is calculated. This process is repeated 100 times with different random sites and the average number of significant p-values were recorded for each interaction. 


\subsubsection{Q Value Analysis}

The p-value signifies the False Positive Rate (FPR), which is the proportion of events truly attributable to random chance (i.e. negatives) that are incorrectly labelled as being statistically significant (i.e. positive). When there are a large number of observations and p-values, the number of expected false positive results can dwarf the number of actually observed significant results. A more stringent measure of significance was required in order to identify the truly statistically significant interactions.

Equation 4.6 shows the mathematical description of the FPR, which is the number of False Positives(FP) divided by the number of True Negatives(TN) and FPs. As discussed, in this case this is a poor measure of significance.

$$
F P R=\frac{F P}{T N+F P}
$$

One method that is used to provide a more stringent threshold and can be used to identify significant results in cases such as this is the q-value [29, 30]. The $\mathrm{q}$-value is related to the p-value but is more useful in this case where there is a large number of observations. The q-value estimates the False Discovery Rate (FDR), which is the proportion of observations considered to be statistically significant (i.e. positive results) that are actually false positives. For 100 significant observations at an FDR of $0.05,5$ of the 100 observations would be expected to be false positives.

$$
F D R=\frac{F P}{T P+F P}
$$

Equation 4.7 shows the mathematical description of FDR, which is the number 
of FPs divided by the number of True Positives(TP) and FPs.

A q-value was calculated for each p-value then various thresholds were selected based on different q-value cutoffs. Each q-value was calculated using the software from $[29,30]$, which is freely available online at:

http://genomics.princeton.edu/storeylab/qvalue/

The q-value method is considered to be less conservative than other corrections for multiple tests, such as the Bonferoni Correction which effectively reduces the p-value threshold for significance by a factor equal to the number of observations $[29,30]$.

\subsubsection{Generating Inputs for Individual PIPE Runs of the SNPdbe Data}

As discussed in Section 4.1.1, a subset of the SNPdbe data was run on PIPE. This data was handled differently from the SKEMPI PIPE run and had significantly more work required to generate the input parameters, for reasons described below. The input parameter generation process is summarized in Figure 4.5 and further details can be found in Appendix A.

Since these mutations mapped directly to Uniprot, the first step is to mutate the wild type protein sequence. This step only requires changing the amino acid at the position specified to the specified mutant amino acid. It is important to note two things about the mutations in SNPdbe. First, all mutations from the SNPdbe are zero indexed and, second, SNPdbe does not specify the wild type amino acid at the mutation position, removing the opportunity of validating that the correct amino acid is being mutated. 
In Figure 4.5 there is a mutation at position four from a glycine to a cysteine. The sequence is mutated at this position, then stored with its wild-type sequence for use in future PIPE runs. The naming convention used is as follows: <protein_name $>$ _ $<$ Wild type amino acid $><$ SNP location $><$ Mutant Amino Acid $>$. This convention is shown in Figure 4.5.

The second step in preparation for a PIPE run is to create the interaction graph file. PIPE uses the interaction graph in the form of a list of known interactions in a file. The known interactions for each mutated protein were removed from the interaction graph in order to simulate the potential effect these SNPs have on the interactome and to provide more conclusive results regarding novel interaction predictions. These known interactions in the interaction graph were removed for both the wild-type and mutant-type PIPE runs of each mutation, effectively creating a leave-one-out test, where all interactions associated with the test sequence are removed from the database prior to testing for the interactions. Figure 4.5 shows two interactions that must be removed.

The final step is the PIPE run itself. Once the interaction graph file has been adjusted in step 2, the PIPE interaction pairs are created. The PIPE algorithm will run on these input pairs. For each mutated protein as well as for the wild type protein, a one-to-all input file was created where PIPE was run on each mutated or wild type protein against all other proteins. In step 3 shown in Figure 4.5 the one-to-all PIPE run is summarized.

As detailed previously in this section each protein mutated with a SNP and the wild type protein requires a separate interaction graph. This, combined with the individual protein pairs on which PIPE will be run, results in 1,276 different input protein pair files to run as well as 1,276 different interaction graphs to be generated. Once these files are generated they all need to be converted to binary 


\begin{tabular}{ll|l|l|l|}
\hline STEP 1: \\
Generate Mutated Protein \\
Sequence
\end{tabular}
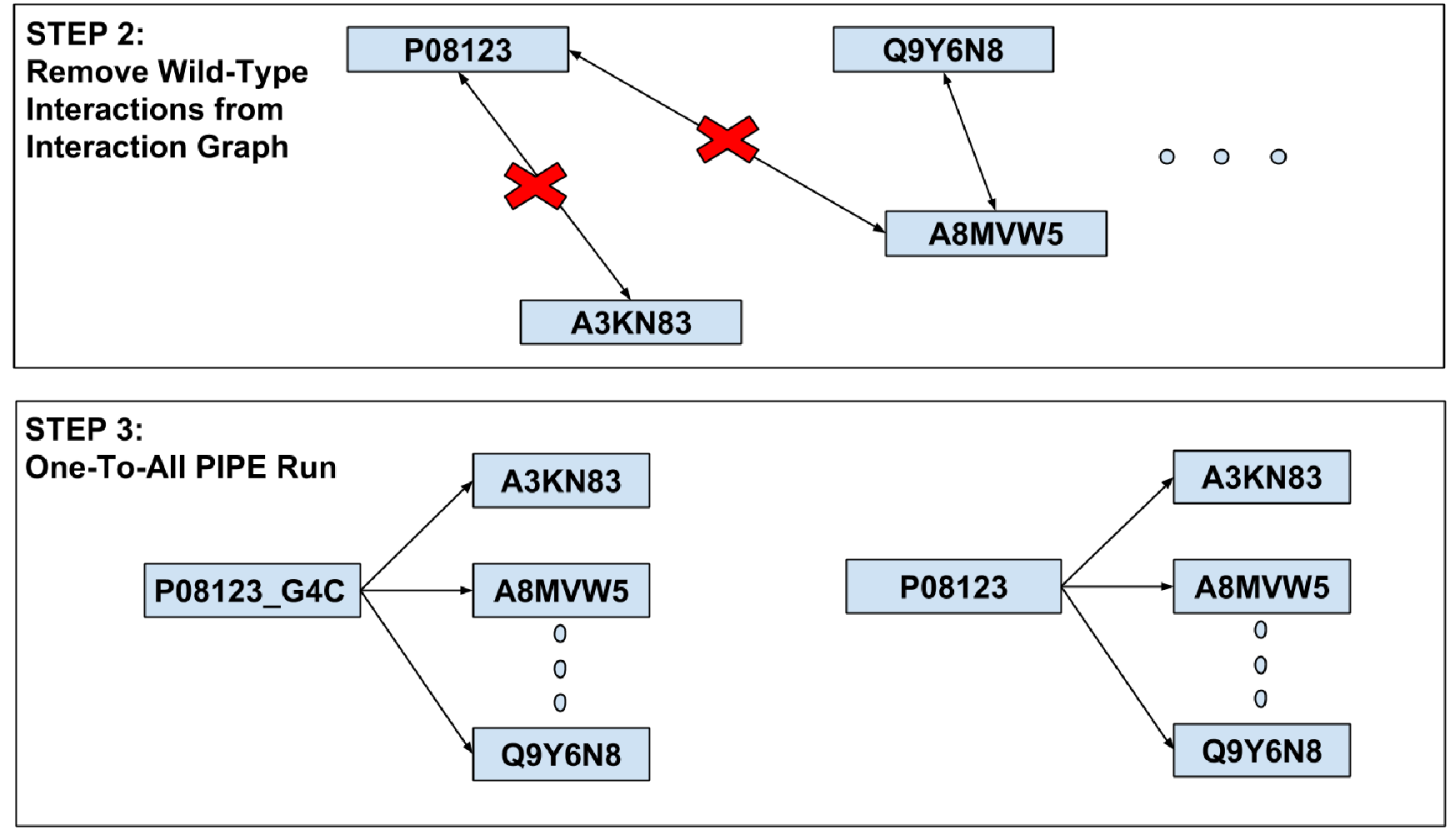

Figure 4.5: Workflow of a PIPE Run for one SNP 


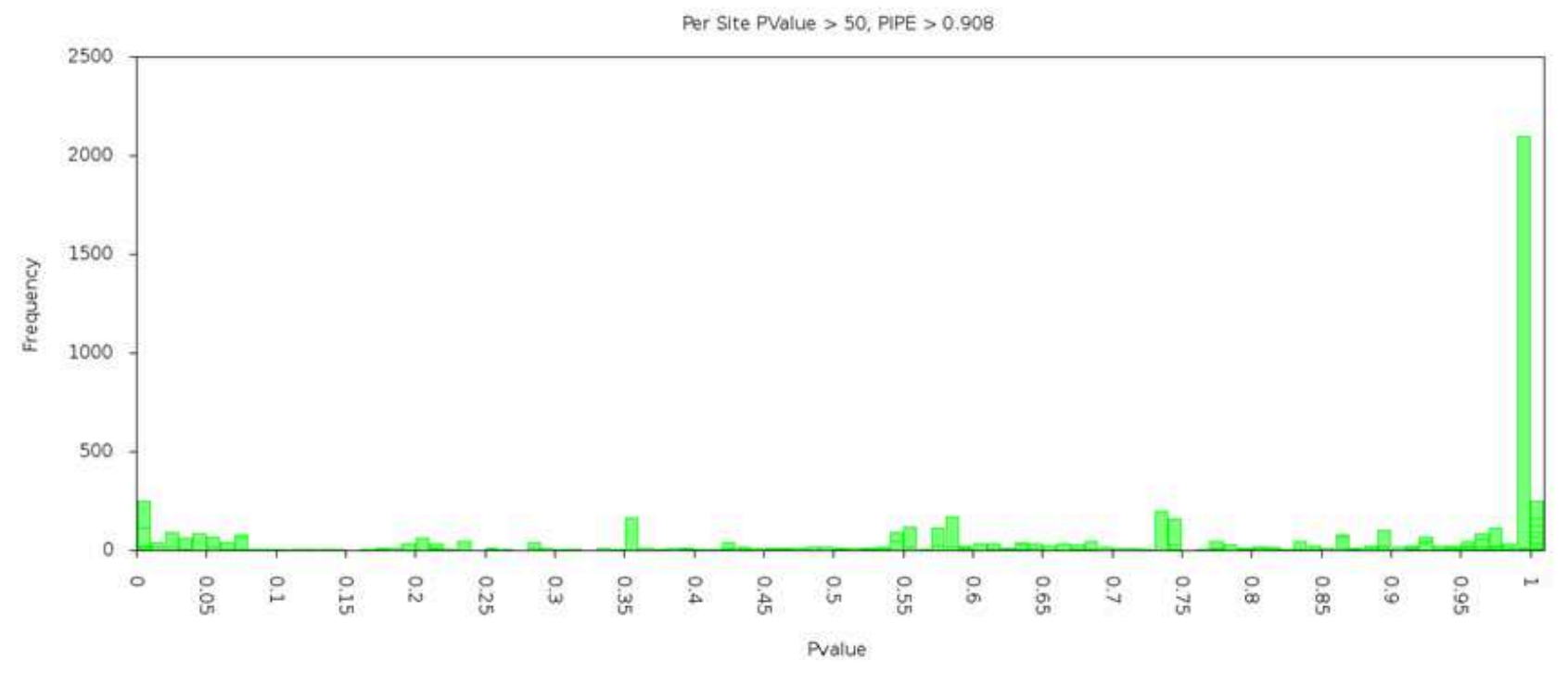

Figure 4.6: Per Site Analysis Histogram

numbers from their original string Uniprot names in order to save space and time. In order to expedite this process, input generation scripts were used, the details of which can be found in Appendix A.

\subsection{Results}

\subsubsection{Per Site Results}

For the results of the per-site analysis detailed in Section 4.2.2, the sites analyzed were limited to those diseases which had 50 or more SNPs to try and have a population sample large enough to remove the effects of random fluctuations. In total there were 13,833 interaction sites analyzed, at the given PIPE threshold of 0.908498. Of these interactions, 1,272 had a p-value of less than 0.05 , which is about $10 \%$ of the total interaction sites analyzed.

Figure 4.6 shows the histogram of the p-values for each PIPE site analyzed. As the Figure 4.6 shows, there is a notable spike of sites that are most definitely 
not significant, however as stated, there were 1,272 that had p-values of less than 0.05, indicating that some PPI sites are significantly enriched with SNPs. This provides evidence that this this subset of interactions may be impacted by diseaseassociated SNPs. These form testable hypotheses that require wet-lab validation experiments and further study.

\subsubsection{Per Protein and Per Interaction Results}

The per-protein, per-interaction analysis examined each mutated protein's interactions. Each of these interactions used all three PIPE sites, from the mutated protein, for the calculations discussed in Section 4.2.3.

In total there were 4,624 protein/interaction pairs which had a PIPE threshold above 0.908498 and more than 50 SNPs occurring in the protein being analyzed. These interactions were analyzed using the method detailed in Section 4.2.3.

From these 4,624 interactions, 337 were statistically significant with a p-value of 0.05 , about $8 \%$ of all interactions. As in previous results, there were significantly more protein/interaction pairs with p-values around 1 than any other value. Figure 4.7 shows a histogram in which the cluster of results around 1, which are not statistically significant, dominates the graph.

While $8 \%$ of the interactions showed statistically significant enrichment of SNPs in PIPE sites, this is not significantly more than would be expected at a p-value of 0.05 . This enrichment in a small number of interactions follows logically since a congenital disease that affects PPIs likely affects a very small subset of the interactome. 


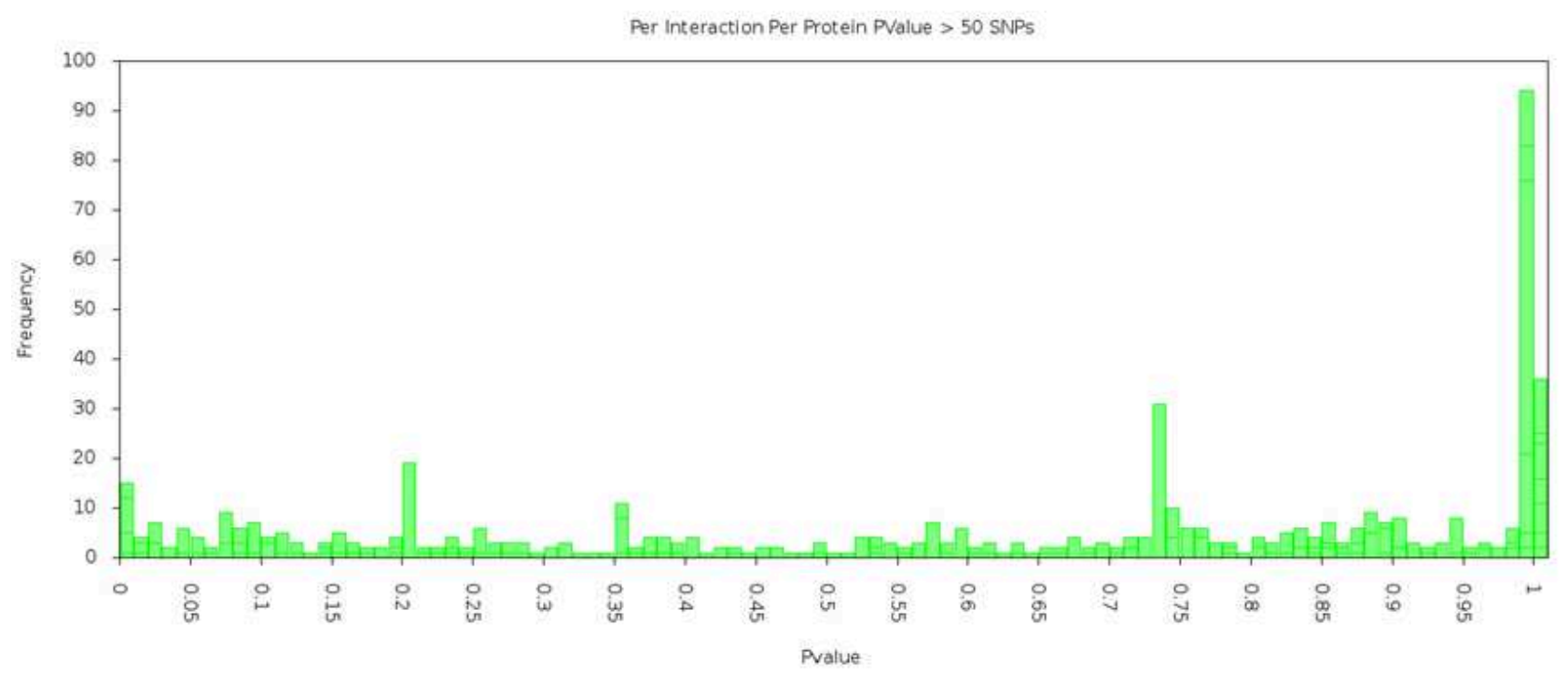

Figure 4.7: Per Protein, Per Interaction Analysis Histogram

\subsubsection{Per Disease Results}

As discussed in the previous sections, there were very few interactions with statistically significant enrichment of SNPs at PIPE interaction sites. While identifying the interactions and SNPs that were significant is valuable biologically, it was desirable to see if a more meaningful pattern could be found. To identify a subset of interactions that have enrichment of SNPs in PIPE predicted interaction sites, the SNPs from the SNPdbe, which are all disease associated, were analyzed on a per disease basis, the details of which can be found in Section 4.2.4. It is important to note all 25,804 disease-related mutations were considered for this experiment.

In total there were 221,983 positive interactions analyzed for the per disease analysis. Each of the 221,983 interactions was assigned a p-value using Equation 4.2 and the set of parameter definitions previously discussed in Section 4.2.4. There were 4,656 statistically significant interactions of the 221,983 were significant at $\mathrm{p}$-value threshold of 0.05 . At that $\mathrm{p}$-value, the expected number of significant results, at random, would be over 10,000, which means that all the 


\begin{tabular}{|c|c|c|}
\hline Q-Value Cutoff & Number of Significant Interactions & Expected False Discoveries \\
\hline \hline 0.05 & 384 & 19.2 \\
\hline 0.01 & 230 & 2.3 \\
\hline 0.001 & 121 & 0.121 \\
\hline
\end{tabular}

Table 4.2: Q-Value Results

statistically significant interactions found could be false positives. This issue was addressed by using the q-value analysis, which more directly relates to false positives and is described in Section 4.2.5.

Of all the random tests performed on each of the 221,293 interactions, only16 diseases and 49 interactions showed any SNP enrichment at the random sites. These 49 interactions represent a small fraction of the 4,656 statistically signficant interactions.

Since the per-disease analysis had such a large population size and relatively few significant interactions, the q-value analysis discussed in Section 4.2.5 was applied in order to determine the significant results.

The results of different q-value cutoffs for the PIPE sites analysis are summarized in Table 4.2 .

The interactions identified from the q-values in Table 4.2 are of high quality with relatively few expected false discoveries. These interactions offer an insight into the disruption to protein interactions caused by the disease with which they are associated. Each line shows the number of significant interactions found as well as the number of false discoveries expected at that particular q-value cutoff. The most promising results appear at a q-value of 0.001 where 121 statistically significant interaction/disease partners were identified with less than 1 expected false discovery. 


\subsubsection{D3JS Graphs}

The histograms above depict the distribution of p-values, which is useful when considering the number of significant results. However, the histograms do not provide any details on the interactions or allow the viewer to see the proteins, interaction sites or disease annotations. To remove the need to examine large text files to extract this detailed information, a web-based graphical presentation was developed using the Javascript library D3 presented in [31].

D3 is a library used for manipulating the Document Object Model (DOM) of HTML pages and facilitates the introduction of useful graphic transitions to create high quality, informative, data-driven images. D3 provides developers with a suite of tools to bind input data to document elements then dynamically transform the elements based on the data. All the elements of a D3 graphic are drawn as a Scalable Vector Graphic(SVG) element, which can then be adjusted to fit the data.

Similar to other document transformation languages like jQuery, the main tool used in D3 is the select command. The objects selected then have some operations performed on them, transforming their appearance according to the operations. For example:

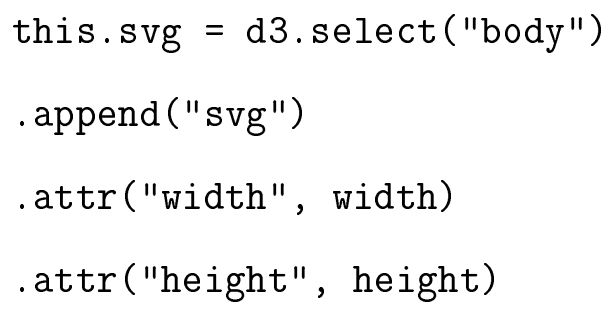

The commands shown select the body element of the HTML page, they then create a single SVG element defined by the parameters width and height. In this case, this command is used to create the SVG container for all other elements of 
the graphic and is usually set to the window size of the browser. The text shown represents one command. Separating each function with a new line makes the code more readable.

Another command that is used select all text in the figure is:

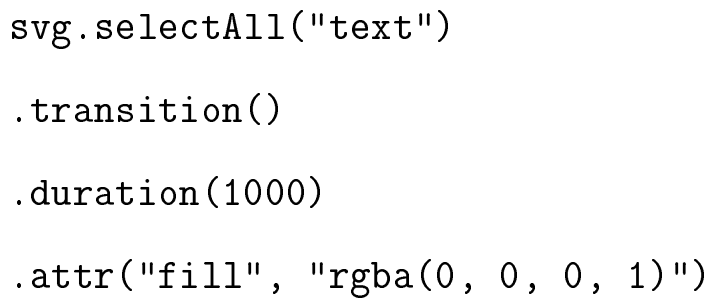

In this case, all text in the SVG element is selected and then transitioned from the original colour to black. The duration parameter takes the duration over which the change occurs in milliseconds.

D3 was used to create a graphic showing each of the high confidence protein interactions and associated data, such as the location of the mutations, location of the interaction sites, protein length and disease information. The proteins depicted are currently all significant interactions at a q-value of 0.05 . This graph is available at the location:

http://cgmlab.carleton.ca/allen/pipe_sites.html

Figure 4.8 shows the proteins without any text. The protein itself is in purple. SNPs are shown as red lines at their respective locations. PIPE predicted interaction sites are shown as three different colours, orange for the first site, pink for the second site and green for the third site. In the web page, all interactions with q-values greater than 0.05 are shown.

When the user hovers over the proteins with the mouse, more information is displayed. In order of appearance, the information displayed is: 
1. Disease Name

2. Mutated protein Uniprot ID

3. The number of SNPs in that protein

4. The protein length in number of amino acids labelled pl in the graphic

Figure 4.9 shows the information displayed after a transition.

It is hoped that this visualization of the results of our research will facilitate future analyses and encourage subsequent wet-lab validation of these testable hypotheses. These graphics can also be used in future web-server development in order to better present results to users.

\subsubsection{SNPdbe PIPE Score Results for PIPE-Sites Data}

The score change on its own did not exhibit any statistically significant change. However, it was hoped that, if analyzed in conjunction with PIPE-Sites, some statistically significant trends would emerge.

The score change data analyzed in this section used the results from the data detailed in Section 4.1.1. This analysis split the PIPE score changes for all mutant interactions into two groups, those where mutations occur in a PIPE site and those that do not occur in a PIPE site. Once the interaction PIPE score changes were separated in this way, the mean score change and standard deviation were calculated for a number of test statistics summarized in Table 4.3.

The first two means, In-Site and Not-in-Site, compare the mean PIPE score change for the interactions where the SNP occurs in the PIPE site and those that do not occur in the PIPE site respectively. 


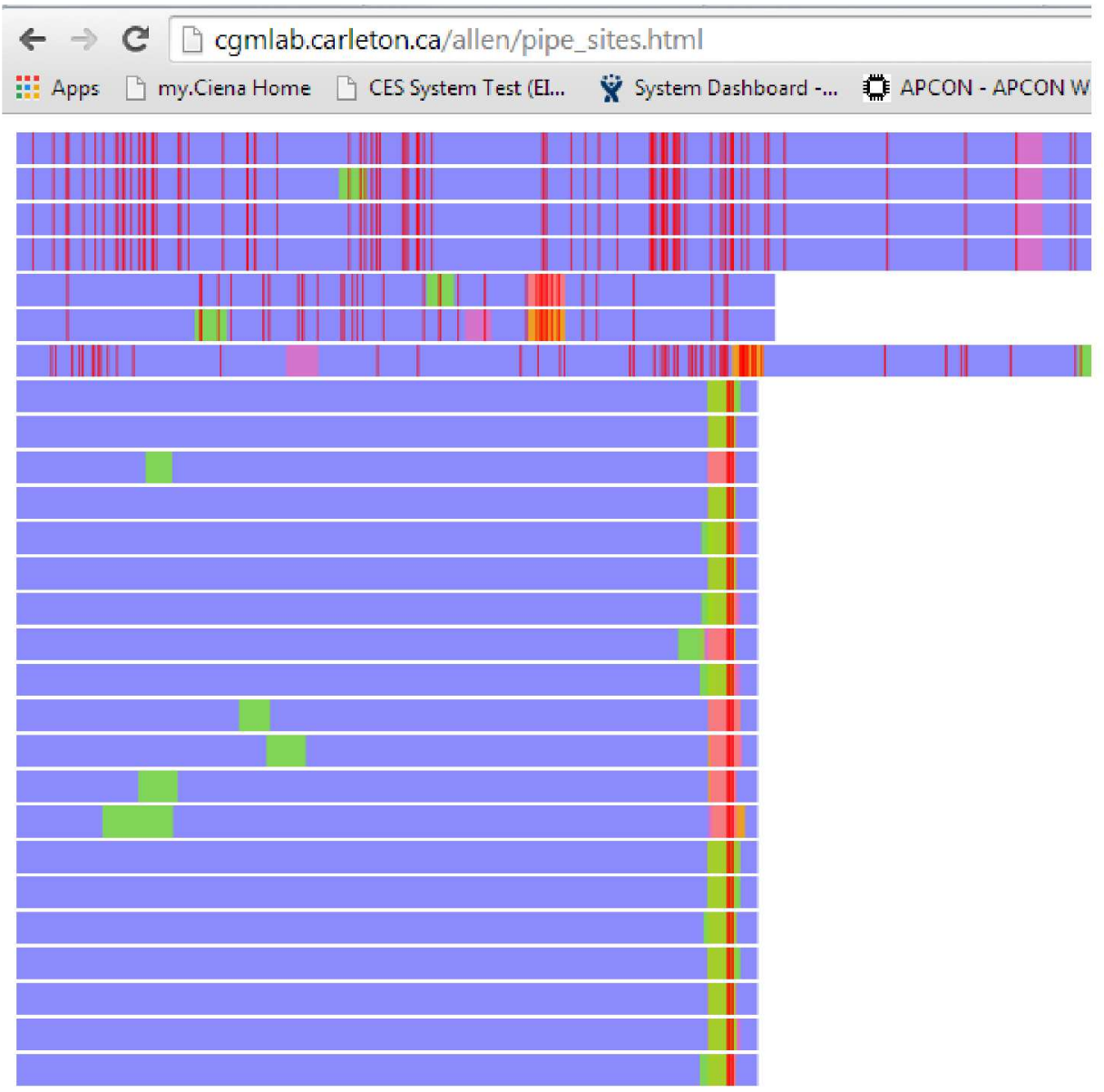

Figure 4.8: D3 Web Graph of Interactions with $\mathrm{Q}<0.05$ 


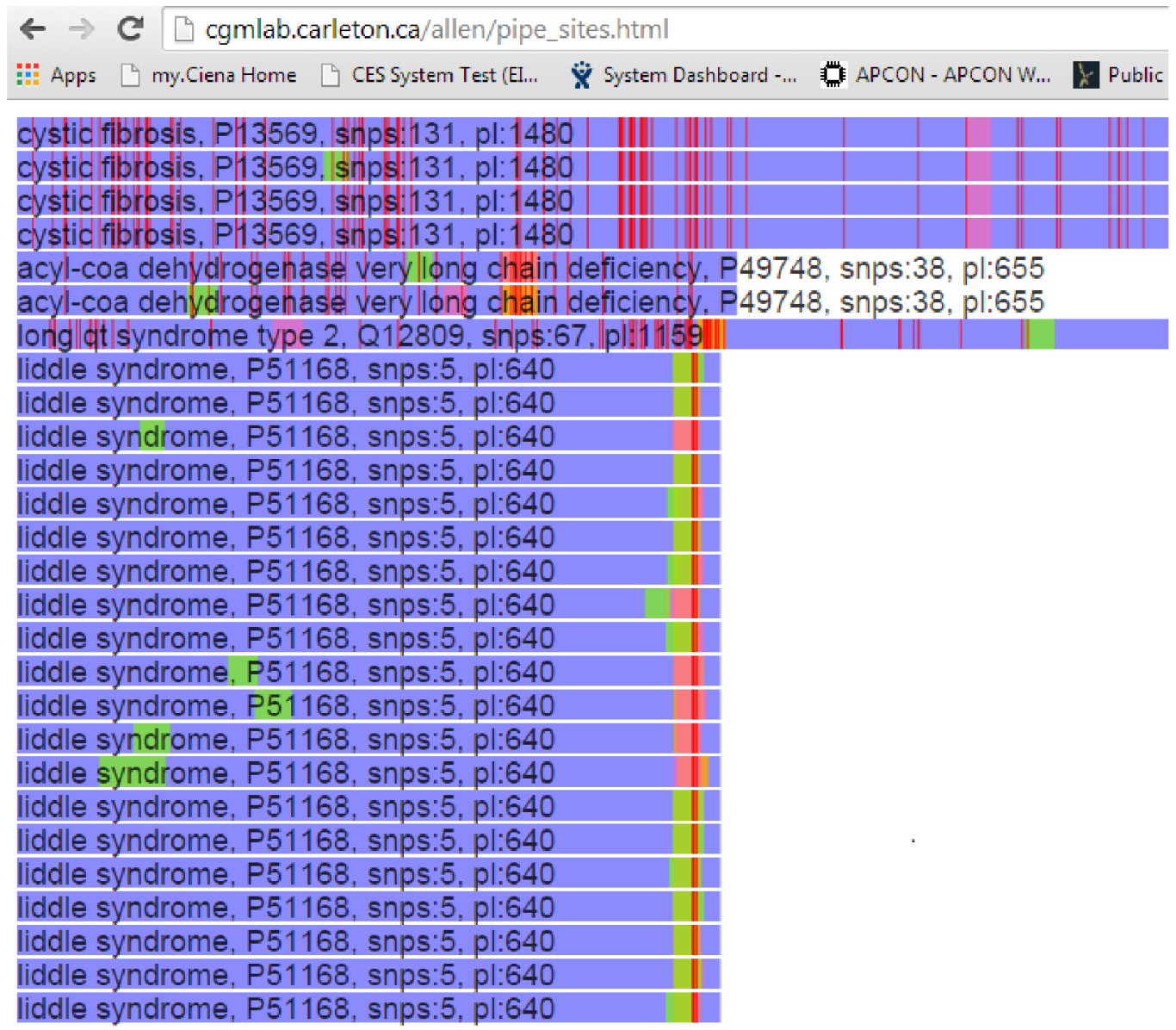

Figure 4.9: D2 Web Graph with Additional Information Shown after a Transition 


\begin{tabular}{|c|c|c|c|}
\hline Filter & Mean & STD & $\mathrm{n}$ \\
\hline \hline In Site & $5.88 \mathrm{E}-05$ & $9.27 \mathrm{E}-04$ & 5224902 \\
\hline Not in Site & $4.63 \mathrm{E}-05$ & $5.80 \mathrm{E}-04$ & 20623173 \\
\hline ABS(In Site) & $2.25 \mathrm{E}-04$ & $9.07 \mathrm{E}-04$ & 5224902 \\
\hline ABS(Not in Site) & $1.41 \mathrm{E}-04$ & $5.64 \mathrm{E}-04$ & 20623173 \\
\hline POS(In Site) & $4.42 \mathrm{E}-04$ & $1.23 \mathrm{E}-03$ & 2005564 \\
\hline POS(Not in Site) & $1.99 \mathrm{E}-04$ & $6.77 \mathrm{E}-04$ & 9712134 \\
\hline Non-zero in Site & $1.92 \mathrm{E}-04$ & $1.20 \mathrm{E}-03$ & 3104649 \\
\hline Non-zero not in Site & $5.88 \mathrm{E}-05$ & $6.52 \mathrm{E}-04$ & 16251267 \\
\hline ABS(Non-zero in Site) & $3.78 \mathrm{E}-04$ & $1.15 \mathrm{E}-03$ & 3104649 \\
\hline ABS(Non-zero not in Site) & $1.80 \mathrm{E}-04$ & $6.30 \mathrm{E}-04$ & 16251267 \\
\hline ABS(POS(Non-zero in Site)) & $4.42 \mathrm{E}-04$ & $1.23 \mathrm{E}-03$ & 2005564 \\
\hline ABS(POS(Non-zero not in Site)) & $1.99 \mathrm{E}-04$ & $6.77 \mathrm{E}-04$ & 9712134 \\
\hline
\end{tabular}

Table 4.3: PIPE Sites Score Change Statistics - All Mutations. Where 'n' is the number of interactions

In order to better compare the magnitude of the changes, the next two means labelled ABS are the means calculated for the absolute values for the PIPE score changes for the in site versus not in site mutations.

Further value can be gained examining only the positive changes to PIPE score. The two means labelled POS only use PIPE score changes that were positive, which signifies a decrease in PIPE score as a results of a SNP.

To further examine the data for magnitude changes, all entries which had no PIPE score change were removed. The Non-zero labelled entries in Table 4.3 use the PIPE score changes for all these non-zero PIPE score changes.

The final four entries are the absolute values for the non-zero and positive score changes, respectively.

It is interesting to note that the average in-site change in PIPE score is approximately 5 times larger than the average score change when a mutation does not occur in a PIPE site. This trend is further supported when looking at rows 5 and 6, when only positive PIPE score changes were considered. This trend indicates 


\begin{tabular}{|c|c|c|c|}
\hline Filter & Mean(C to W) & STD $(\mathrm{C}$ to W $)$ & $\mathrm{n}$ \\
\hline \hline In Site & $3.99 \mathrm{E}-04$ & $1.11 \mathrm{E}-03$ & 304955 \\
\hline Not in Site & $1.37 \mathrm{E}-04$ & $6.70 \mathrm{E}-04$ & 1600707 \\
\hline ABS(In Site) & $4.52 \mathrm{E}-04$ & $1.09 \mathrm{E}-03$ & 304955 \\
\hline ABS(Not in Site) & $1.81 \mathrm{E}-04$ & $6.60 \mathrm{E}-04$ & 1600707 \\
\hline POS(In Site) & $6.41 \mathrm{E}-04$ & $1.18 \mathrm{E}-03$ & 137401 \\
\hline POS(Not in Site) & $2.63 \mathrm{E}-04$ & $7.83 \mathrm{E}-04$ & 1019477 \\
\hline Non zero in Site & $5.04 \mathrm{E}-04$ & $1.25 \mathrm{E}-03$ & 172660 \\
\hline Non zero not in Site & $1.75 \mathrm{E}-04$ & $7.54 \mathrm{E}-04$ & 1312147 \\
\hline ABS(Non zero in Site) & $5.86 \mathrm{E}-04$ & $1.21 \mathrm{E}-03$ & 172660 \\
\hline ABS(Non zero not in Site) & $2.32 \mathrm{E}-04$ & $7.39 \mathrm{E}-04$ & 1312147 \\
\hline ABS(POS(In Site) & $6.41 \mathrm{E}-04$ & $1.18 \mathrm{E}-03$ & 137401 \\
\hline ABS(POS(not in Site)) & $2.63 \mathrm{E}-04$ & $7.83 \mathrm{E}-04$ & 1019477 \\
\hline
\end{tabular}

Table 4.4: PIPE Sites Score Change Statistics - C to W Mutations. Where 'n' is the number of interactions

that when there is a SNP in a PIPE-Site the results PIPE score change is larger than when the SNP is in another location on the protein.

Once again, there were very small changes to PIPE scores regardless of the metric used. In order to provide a sanity check to make sure that even the most disruptive SNPs do not cause large changes to PIPE, only interactions that had a C (Cysteine) mutated to a W (Tryptophan), which has the largest PAM score of all substitutions possible, were examined to see if this results in a larger change in PIPE score. Table 4.4 summarizes the score change for the interactions which had only a $\mathrm{C}$ to $\mathrm{W}$ mutation. The same filters were used as for the overall score change calculations.

When analyzing the the results, there is not a significant change, in magnitude, to PIPE score for any of the various filters applied to the data. The means for each in-site/not-in-site pair can be quantitatively compared and any significant difference identified.

In comparing the in-site/not-in-site mean values, to determine statistical sig- 
nificance, a null hypothesis was determined. In this case the null hypothesis is that there is no difference between the two sample means. The condition stated mathematically is:

$$
h_{0}: \mu_{1}-\mu_{2}=0
$$

where $\mu_{1}$ is the mean value of the PIPE score change for in-site interactions and $\mu_{2}$ is the mean value of the PIPE score change for not-in-site interactions.

Calculating the $\mathrm{Z}$ value for the test statistic $\mu_{1}-\mu_{2}$ will quantify the decision on whether the null hypothesis can be rejected.

To calculate the $\mathrm{Z}$ value, the following formula was used:

$$
z=\frac{\bar{x}_{1}-\bar{x}_{2}}{\sqrt{\frac{\sigma_{1}^{2}}{n_{1}}+\frac{\sigma_{2}^{2}}{n_{2}}}}
$$

Using this formula, a $\mathrm{Z}$ value was calculated comparing each pair of means for each filter, that is, row 1 and row 2 were compared, row 3 and row 4 and so on. Table 4.5 summarizes the results for each mean score-change pair. The results for the $\mathrm{C}$ to $\mathrm{W}$ mean score change pairs had similarly high $\mathrm{Z}$ values when compared. These high $\mathrm{Z}$ values indicate the difference between the two means is statistically significant and the null hypothesis can be rejected with a very high degree of confidence.

Based on the results shown in Table 4.5, all metrics exhibit a statistically significant difference in PIPE score change between the in-site/not-in-site cases. The most significant of these differences is the case of the non-zero measurement where all null change to PIPE score are removed. This is as expected since removing all of these null changes further emphasizes any changes there are to PIPE score. 


\begin{tabular}{|c|c|}
\hline Filter & Z Value \\
\hline \hline None & 159 \\
\hline ABS & 200 \\
\hline POS & 270 \\
\hline Non-zero & 296 \\
\hline ABS, None-zero & 296 \\
\hline ABS, POS & 270 \\
\hline
\end{tabular}

Table 4.5: Z Value Mean Comparison for Score Change of All SNPdbe Mutations

While these results are statistically significant and show that SNPs do cause a change to PIPE score it should be noted that, due to the size of the standard deviation relative to the mean, the PIPE score change may not be useful feature for predicting SNP-induced interactome changes.

\subsection{Conclusion}

PIPE-Sites appears to be a viable feature that can be used to identify potential SNP-induced interactome changes. The disease SNPs from SNP-dbe showed statistically significant enrichment at PIPE sites. Also, a subset of disease-causing SNPs that were run on PIPE caused a statistically significant score change based on location in relation to PIPE sites.

There are other methods that predict the effect SNPs have on proteins however theses tools generally use 3D structure data, which limits their use on the interactome scale. These methods also look at the functional [25] or structural $[23,25]$ effects, rather than effects on the interactome itself.

Currently there are no datasets that present the data needed to validate the interactions and diseases identified in this section.

In order to validate these findings through wet-lab experimentation, the PIPE sites which showed statistical enrichment and their associate interactions could 
be replicated. The mutated and wild-type interaction partners would then need to be measured for their respective affinities. If the mutated proteins no longer interacted or showed a large change in affinity, this would validate that SNP enrichment at PIPE sites can be used predict interactome changes.

The validation of some of the diseases identified through SNP-enrichment can be made by examing the diseases-specific research. Some of the diseases identified are known to function by disrupting PPIs, like cystic fibrosis [32], breast cancer [33], long QT syndrome [34] and colorectal adenomas [18]. This evidence supports the hypothesis that theses diseases function in part by disrupting critical protein interactions. 


\section{$5 \quad$ Using PIPE-Sites to Identify Interactome Changes Caused by Known Co-occurring SNPs from 1KG Genotypes}

Based on the results from the SNPdbe disease-causing mutations, collections of SNPs do show some enrichment at PIPE sites and mutations in PIPE sites do cause a statistically significant change to PIPE score, albeit small in magnitude. Thus far, the sets of SNPs analyzed so far do not necessarily co-occur.

Genotypes, sets of observed co-occurring SNPs, were examined to further investigate the potential enrichment of SNPs at PIPE-Sites. Statistically significant enrichment of SNPs at PIPE-Sites could be used to identify the interactome changes due to a particular genotype, which another step towards personalized interactome change prediction.

\subsection{Data Source: 1000 Genomes}

The 1000 Genomes (1KG) project was started in September 2007 with the aim of sequencing a large number of individuals from various representative populations across the globe. The goal in acquiring this data was to characterize mutations as well as to provide the research community with well-sequenced genomic data at no cost. Modern high-throughput gene sequencing technology is able to elucidate a gene sequence by taking a large number of short DNA reads of less than 50 base pairs each. The average read depth of a sample indicates the number of reads each base pair from the target sample is part of, on average. A higher read depth indicates better quality estimates of an individual's genome.

There were a number of phases to the $1 \mathrm{KG}$ project including low coverage 
sequencing of about 2500 individuals (2-6x read depth), higher coverage exontargeted sequencing $(50 \mathrm{x}-100 \mathrm{x}$ read depth) which expanded on the previous low coverage results and included more individuals, and finally analysis of genetic inheritance in families.

The collection of mutations found in specific samples provide an opportunity to measure PIPE-Sites' performance on known sets of SNPs that are shown to co-occur in nature. In order to gauge this performance, 64 sets of SNPs from 32 individuals in the GBR population were analyzed. Each individual had genotype information provided from the $1 \mathrm{KG}$ project and consequently had two sets of cooccurring SNPs available, from each of the respective copies of each chromosome. Samples are labelled as follows:

$<1$ KG Sample ID $>$ < Genotype: h1 or h2 $>$

\subsubsection{Sequence Alignment}

Each individual from the $1 \mathrm{KG}$ project had a multitude of short DNA sequence reads that are the result of next-generation sequence technologies DNA sequencing method. Each of these short sequence reads must be aligned to the reference genome in order to form a consensus genotype for each individual. This can be done manually using computational alignment tools like the SAMtools library, however the $1 \mathrm{KG}$ project provides aligned sequence reads as well as genotype information [35]. These aligned sequence reads were aligned using either the Burrows-Wheeler Alignment algorithm or the Mosaik alignment algorithm various research institutions, the details are available in [5]. 


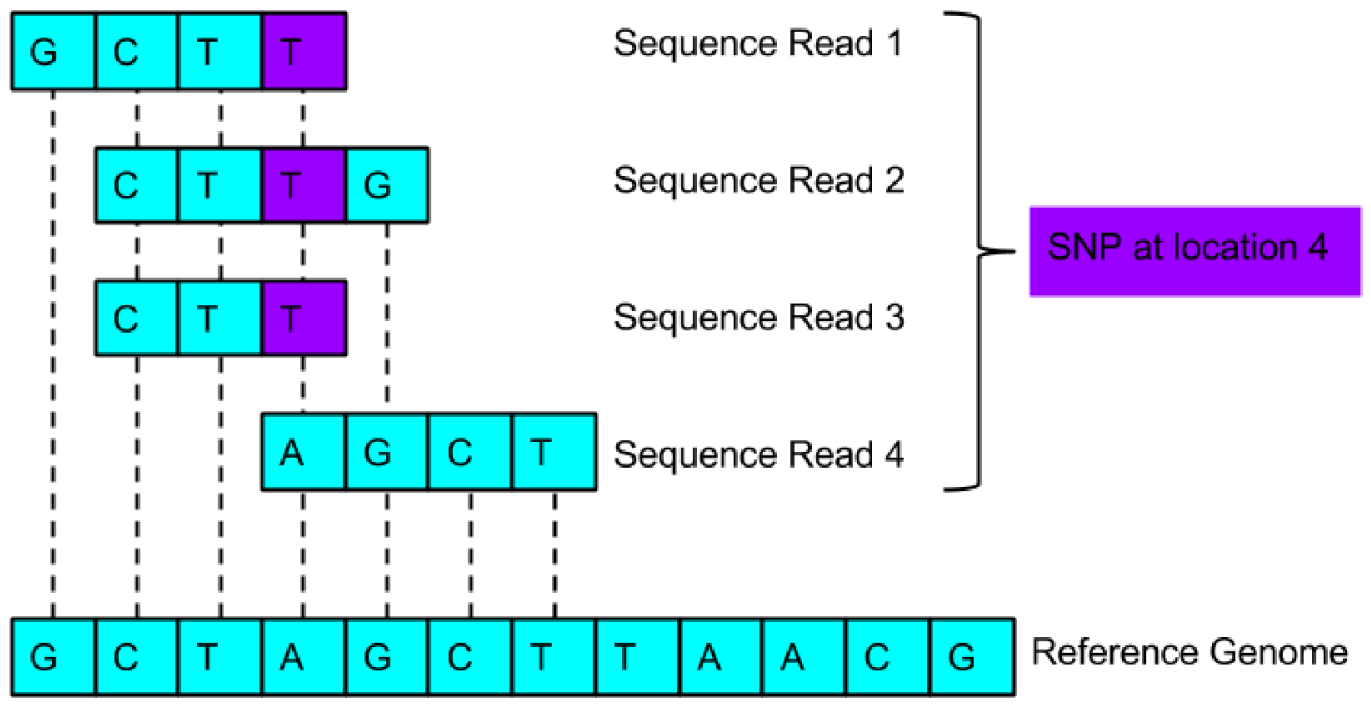

Figure 5.1: SNP Prediction from Multiple DNA Sequence Reads

\subsubsection{SNP Calling}

Once the sequence reads for an individual are aligned, differences between the individual's reads and the reference genome must be documented. DNA sequencing is an inexact science and due to factors like varying read-quality, lower read depths and the fact that humans have two copies of each gene, a difference between a sequence read and the reference genome is not always a true SNP [35].

Figure 5.1 shows an example of 4 sequence reads for an individual that are mapped to the reference genome. As can be seen, there is a potential SNP at position 4 . Three of the four sequence reads have an alternate base pair at location 4, a thymine instead of an adenine, which is highlighted in purple. Since the majority of the sequence reads show an alternate base at location 4 , this SNP would be documented as part of the individual's genotype.

In addition to looking at the consensus for each base pair in the sequence reads, there is also a quality score for each sequence read which is also incorporated into SNP calling for the individuals in the $1 K G$ project. 


\subsubsection{SNP Acquisition from $1 \mathrm{KG}$}

The $1 \mathrm{KG}$ project provides a condensed file containing high confidence SNPs for all individuals. The condensed file is in the Variant Call Format(VCF) which can be easily analyzed in a number of ways using the software toolkit vcf-tools. The condensed SNP file can be analyzed for SNPs only occurring in a specific individual using the vcf-tools command:

vcf-subset $-c<$ individual_id $><$ condensed_VCF_file $>\mid$ bgzip $-c><$ output $>$

Due to the size of this file $(\sim 62 \mathrm{G})$, extracting a subset for one individual takes on average 4.5 hours. Each individual's SNPs were acquired from the condensed VCF file using the command shown above and further subdivided into each individual's genotype.

\subsubsection{SNP Conversion to Amino Acid Substitutions}

Since PIPE deals with protein sequences, not DNA sequences, the genotypes of the individuals selected from the $1 \mathrm{KG}$ project had to be converted to the protein level. This process involved mapping the areas of DNA that code for proteins, called exons, to their respective protein sequences and then analyzing the location and effect of all the SNPs. This process is also known as gene annotation. Fortunately there is a free software package, ANNOVAR, available that accepts a VCF file as input and annotates the SNPs therein, providing information on the effects, including amino acid substitution, each SNP has [36].

The SNP information produced by ANNOVAR appears as a colon delimited field that has the following information, in order of appearance:

1. Gene Name

2. RefSeq Protein ID 


\begin{tabular}{|c|c|c|}
\hline Line Number & Type of SNP & SNP Information \\
\hline \hline 357 & Synonymous & NOC2L:NM_015658:exon9:c.A918G:p.E306E \\
\hline 411 & Non-Synonymous & PERM1:NM_001291367:exon5:c.T2048C:p.V683A \\
\hline
\end{tabular}

Table 5.1: ANNOVAR Output File

3. Exon Number

4. DNA Substitution in the form: c. $<$ wild type base $><$ DNA substitution location in gene $><$ mutant base $>$

5. Protein Amino Acid Substitution in the form:

p. $<$ wild type amino acid $><$ substitution location in protein $><$ mutant amino acid $>$

ANNOVAR did not directly map to the Uniprot protein ID so RefSeq protein IDs were used, then mapped to Uniprot using a mapping file from the Uniprot website. The mapping file provided by Uniprot was generated by uploading all the RefSeq IDs at the URL:

\section{http: //www.uniprot.org/uploadlists/}

The non-synonymous SNP shown in line 2 of Table 5.1 would first be mapped to its RefSeq counterpart and then mapped to the Uniprot protein and sequence. The amino acid at the mutation location of the Uniprot protein would then be compared to the wild type amino acid, V. If the amino acids match, the mapping would be recorded as a success. Any mutations for which the wild amino acid did not match the Uniprot entry were removed and not part of any analysis.

Tables 5.4 and 5.5 summarize the data for each individual analyzed from the GBR population. Shown are the total mutations, the mutations that map to Uniprot and the mutations that were not mapped for various reasons. As shown, 
on average $86 \%$ of the mutations mapped to Uniprot proteins. The last line of table 5.5 shows the mean values for each respective column.

\subsection{PIPE Sites 1KG Results}

A better indication of the strength of PIPE-Sites as a SNP-induced interactome change indicator would be to search for enrichment of co-occurring SNPs in PIPE sites. To this end, the SNP sets from the 1KG project discussed in Section 5.1 were analyzed for any enrichment at PIPE-predicted interaction sites. Similar to the disease analysis performed, there is strong evidence that there is enrichment of SNPs at some PIPE predicted interaction sites.

Each set of SNPs summarized in Tables 5.2 and 5.3 was analyzed to search for interactions that may have SNP enrichment at PIPE predicted interaction sites. In order to accomplish this, a similar analysis to the per site analysis described in 4.2.2 was performed, however only the first and most significant PIPE-Site was used.

For the analysis, every protein with more than 5 SNPs was analyzed. 5 SNPs was selected as the threshold since any less can be affected too much by randomness, while at the threshold of 5 still leaves many proteins that can be included in the analysis. Each protein with more than 5 SNPs had all interaction sites with positive interaction partners analyzed and a p-value calculated using the same calculation outlined in Section 4.2.2.

One case which this method does not account for was when both interaction partners have more than 5 SNPs. In this case the calculation for p-value used was the same as that for the per-interaction analysis described in Section A.6.

Both sets of p-values, whether there were mutations in one or both interaction 


\begin{tabular}{|c|c|c|c|}
\hline Sample & Total & Mapped to Uniprot & Percentage \\
\hline \hline HG00098_h1 & 6396 & 5487 & 0.857879925 \\
\hline HG00098_h2 & 6775 & 5874 & 0.86701107 \\
\hline HG00100_h1 & 6404 & 5472 & 0.854465959 \\
\hline HG00100_h2 & 6755 & 5863 & 0.867949667 \\
\hline HG00106_h1 & 6630 & 5667 & 0.854751131 \\
\hline HG00106_h2 & 6765 & 5757 & 0.850997783 \\
\hline HG0011__h1 & 6515 & 5647 & 0.866768995 \\
\hline HG0011__h2 & 6687 & 5758 & 0.861073725 \\
\hline HG00114_h1 & 6504 & 5609 & 0.862392374 \\
\hline HG00114_h2 & 6720 & 5762 & 0.857440476 \\
\hline HG00116_h1 & 6717 & 5746 & 0.855441417 \\
\hline HG00116_h2 & 6780 & 5869 & 0.865634218 \\
\hline HG00117_h1 & 6750 & 5815 & 0.861481481 \\
\hline HG00117_h2 & 6806 & 5821 & 0.855274758 \\
\hline HG00118_h1 & 6390 & 5507 & 0.861815336 \\
\hline HG00118_h2 & 6669 & 5786 & 0.867596341 \\
\hline HG00119_h1 & 6796 & 5854 & 0.861389052 \\
\hline HG00119_h2 & 6972 & 5991 & 0.85929432 \\
\hline HG00120_h1 & 6624 & 5775 & 0.87182971 \\
\hline HG00120_h2 & 6801 & 5885 & 0.865313924 \\
\hline HG00122_h1 & 6442 & 5538 & 0.85967091 \\
\hline HG00122_h2 & 6746 & 5845 & 0.866439371 \\
\hline HG00123_h1 & 6278 & 5394 & 0.859190825 \\
\hline HG00123_h2 & 6549 & 5598 & 0.85478699 \\
\hline HG00124_h1 & 6972 & 5992 & 0.859437751 \\
\hline HG00124_h2 & 7203 & 6132 & 0.851311953 \\
\hline HG00126_h1 & 6532 & 5567 & 0.852265769 \\
\hline HG00126_h2 & 6767 & 5795 & 0.856361756 \\
\hline HG00131_h1 & 6738 & 5739 & 0.85173642 \\
\hline HG00131_h2 & 7001 & 5948 & 0.849592915 \\
\hline HG00141_h1 & 6412 & 5590 & 0.87180287 \\
\hline HG00141_h2 & 6795 & 5851 & 0.861074319 \\
\hline
\end{tabular}

Table 5.2: 1KG Sample Data Summary 1 


\begin{tabular}{|c|c|c|c|}
\hline Sample & Total & Mapped to Uniprot & Percentage \\
\hline \hline HG00142_h1 & 6652 & 5726 & 0.860793746 \\
\hline HG00142_h2 & 6791 & 5851 & 0.861581505 \\
\hline HG00143_h1 & 6435 & 5574 & 0.866200466 \\
\hline HG00143_h2 & 6733 & 5828 & 0.865587405 \\
\hline HG00144_h1 & 6171 & 5288 & 0.85691136 \\
\hline HG00144_h2 & 6243 & 5361 & 0.858721768 \\
\hline HG00145_h1 & 6370 & 5534 & 0.868759812 \\
\hline HG00145_h2 & 6622 & 5724 & 0.864391423 \\
\hline HG00146_h1 & 6768 & 5787 & 0.855053191 \\
\hline HG00146_h2 & 7007 & 6001 & 0.856429285 \\
\hline HG00147_h1 & 6465 & 5486 & 0.848569219 \\
\hline HG00147_h2 & 6823 & 5899 & 0.8645757 \\
\hline HG00148_h1 & 6390 & 5495 & 0.859937402 \\
\hline HG00148_h2 & 6745 & 5809 & 0.861230541 \\
\hline HG00149_h1 & 6406 & 5455 & 0.851545426 \\
\hline HG00149_h2 & 6850 & 5879 & 0.858248175 \\
\hline HG00150_h1 & 6273 & 5355 & 0.853658537 \\
\hline HG00150_h2 & 6509 & 5586 & 0.858196344 \\
\hline HG00151_h1 & 6370 & 5481 & 0.86043956 \\
\hline HG00151_h2 & 6476 & 5571 & 0.860253243 \\
\hline HG00152_h1 & 6537 & 5630 & 0.861251339 \\
\hline HG00152_h2 & 6864 & 5878 & 0.856351981 \\
\hline HG00153_h1 & 6326 & 5452 & 0.861840025 \\
\hline HG00153_h2 & 6787 & 5826 & 0.858405776 \\
\hline HG00156_h1 & 6507 & 5640 & 0.866758875 \\
\hline HG00156_h2 & 6666 & 5715 & 0.857335734 \\
\hline HG00158_h1 & 6538 & 5654 & 0.864790456 \\
\hline HG00158_h2 & 6982 & 5978 & 0.856201661 \\
\hline HG00159_h1 & 6625 & 5688 & 0.858566038 \\
\hline HG00159_h2 & 6871 & 5931 & 0.863193131 \\
\hline HG00160_h1 & 6366 & 5497 & 0.86349356 \\
\hline HG00160_h2 & 6682 & 5763 & 0.862466327 \\
\hline All_ & 6636.578125 & 5707.4375 & 0.860018946 \\
\hline
\end{tabular}

Table 5.3: 1KG Sample Data Summary 1 
partners, were recorded separately then compared.

In order to further validate that the observed enrichment of SNPs at certain interaction sites, a randomized test was performed as well on each interaction examined, in the same way described in Section 4.2.4. Only one interaction from individual HG00144_h2 showed any random SNP enrichment.

Tables 5.4 and 5.5 summarize the number of sites analyzed as well as the number of significant interactions found. On average, approximately $18 \%$ of single mutation sites were significant and $19 \%$ of double mutations sites were significant, at a p-value of 0.05 .

For each genotype there were two files generated. The interactions and their associated p-values which had a single protein with mutations were contained in one file and the other file contained interactions and the associated p-values for those interactions in which both proteins had mutations. Each file had 3 columns, the first two corresponding to the interacting proteins and the third to the calculated p-value.

Overall a single file containing all unique interactions predicted to be affected by at least one genotype was generated. This file presents all statistically significant interactions found. Some interactions may be repeated, for those cases, the lowest p-value for the interaction was recorded. In total, there were 2,726 unique statistically significant interactions of a possible 8,121. Since each genotype had, on average about 2,000 statistically significant interactions and there were only 2,726 unique interactions, a large number of interactions must have been shared by multiple genotypes. These interactions represent the first step in quantifying the effects co-occurring SNPs have on the interactome. These SNPs, which presumably are not as disruptive as the disease causing SNPs from SNPdbe, still exhibit some enrichment at PIPE sites. This further proves that PIPE-Sites can 
be used as a tool to identify changes to the interactome caused, not only by disease causing SNPs, but more common SNPs from patient's genotypes.

These interactions can be examined and used by biologists for wet lab experiments examining the changes caused by co-occurring SNPs to the interactome. The list of interactions is publicly available for download at:

http://cgmlab.carleton.ca/allen/interaction.list

\subsection{Conclusion}

Analysis of congenital disease-causing SNPs in Section 4 resulted in the identification of potential SNP-induced interactome changes. These disease SNPs do not necessarily occur together, so we decided to examine sets of known genotypes from the $1 \mathrm{KG}$ project, in conjunction with PIPE-Sites. A list of interactions from these genotypes was generated which show statistically significant enrichment of SNPs in PIPE-Sites. This list of interactions represents the potential interactome changes caused by the genotypes from the $1 \mathrm{KG}$ project. These interactome changes are publicly available from the CGM server at Carleton University.

In order to validate the findings here, each interaction would need to be tested in the lab. Ideally, the two proteins in any of the list of affected interactions would be shown to interact without mutation and the protein affinity would be masured. The mutations would then be performed and the affinity and interaction between the two proteins would then be measured. To develop a more robust experiment, a third case where a number of random mutations were performed could also be quantified to see if there is a statistically significant difference between the interaction change when mutations occur randomly. 


\begin{tabular}{|c|c|c|c|c|}
\hline Sample & Total Single & Total Double & Significant Single & Significant Double \\
\hline HG00098_h1 & 1776 & 431 & 319 & 87 \\
\hline HG00098_h2 & 2166 & 533 & 342 & 78 \\
\hline HG00100_h1 & 1056 & 356 & 209 & 49 \\
\hline HG00100_h2 & 1511 & 361 & 342 & 103 \\
\hline HG00106_h1 & 1745 & 522 & 317 & 92 \\
\hline HG00106_h2 & 1338 & 423 & 309 & 83 \\
\hline HG00112_h1 & 1236 & 290 & 277 & 57 \\
\hline HG00112_h2 & 1454 & 387 & 327 & 68 \\
\hline HG00114_h1 & 1110 & 278 & 277 & 51 \\
\hline HG00114_h2 & 1133 & 279 & 286 & 73 \\
\hline HG00116_h1 & 1583 & 388 & 250 & 65 \\
\hline HG00116_h2 & 1640 & 445 & 261 & 64 \\
\hline HG00117_h1 & 1444 & 366 & 322 & 77 \\
\hline HG00117_h2 & 1578 & 338 & 269 & 68 \\
\hline HG00118_h1 & 1032 & 204 & 269 & 65 \\
\hline HG00118_h2 & 1567 & 395 & 277 & 57 \\
\hline HG00119_h1 & 1682 & 396 & 312 & 64 \\
\hline HG00119_h2 & 1719 & 451 & 331 & 101 \\
\hline HG00120_h1 & 2177 & 629 & 357 & 95 \\
\hline HG00120_h2 & 1985 & 731 & 380 & 121 \\
\hline HG00122_h1 & 1415 & 382 & 266 & 69 \\
\hline HG00122_h2 & 1720 & 560 & 317 & 103 \\
\hline HG00123_h1 & 1525 & 318 & 271 & 67 \\
\hline HG00123_h2 & 1581 & 410 & 310 & 70 \\
\hline HG00124_h1 & 1822 & 467 & 344 & 111 \\
\hline HG00124_h2 & 1618 & 279 & 293 & 73 \\
\hline HG00126_h1 & 1775 & 398 & 299 & 79 \\
\hline HG00126_h2 & 2121 & 648 & 403 & 114 \\
\hline HG00131_h1 & 1950 & 600 & 288 & 84 \\
\hline HG00131_h2 & 1632 & 509 & 295 & 72 \\
\hline HG00141_h1 & 1468 & 376 & 290 & 69 \\
\hline HG00141_h2 & 2155 & 604 & 329 & 104 \\
\hline
\end{tabular}

Table 5.4: 1KG PIPE-Sites Analysis Results Summary 1 


\begin{tabular}{|c|c|c|c|c|}
\hline Sample & Total Single & Total Double & Significant Single & Significant Double \\
\hline HG00142_h1 & 1604 & 459 & 285 & 82 \\
\hline HG00142_h2 & 1703 & 410 & 283 & 74 \\
\hline HG00143_h1 & 1294 & 346 & 238 & 48 \\
\hline HG00143_h2 & 1564 & 416 & 395 & 102 \\
\hline HG00144_h1 & 1217 & 271 & 235 & 66 \\
\hline HG00144_h2 & 1331 & 383 & 305 & 102 \\
\hline HG00145_h1 & 1158 & 297 & 295 & 71 \\
\hline HG00145_h2 & 1549 & 360 & 350 & 97 \\
\hline HG00146_h1 & 1873 & 509 & 328 & 104 \\
\hline HG00146_h2 & 1912 & 583 & 305 & 86 \\
\hline HG00147_h1 & 1580 & 417 & 282 & 88 \\
\hline HG00147_h2 & 1668 & 428 & 275 & 86 \\
\hline HG00148_h1 & 1664 & 328 & 283 & 61 \\
\hline HG00148_h2 & 1911 & 557 & 309 & 89 \\
\hline HG00149_h1 & 1824 & 546 & 373 & 97 \\
\hline HG00149_h2 & 1778 & 440 & 401 & 94 \\
\hline HG00150_h1 & 1436 & 357 & 333 & 90 \\
\hline HG00150_h2 & 1852 & 471 & 301 & 75 \\
\hline HG00151_h1 & 1123 & 240 & 234 & 51 \\
\hline HG00151_h2 & 1165 & 339 & 201 & 43 \\
\hline HG00152_h1 & 2056 & 523 & 345 & 97 \\
\hline HG00152_h2 & 2120 & 609 & 323 & 103 \\
\hline HG00153_h1 & 1211 & 379 & 234 & 61 \\
\hline HG00153_h2 & 1969 & 348 & 377 & 72 \\
\hline HG00156_h1 & 1440 & 368 & 267 & 81 \\
\hline HG00156_h2 & 1587 & 405 & 284 & 65 \\
\hline HG00158_h1 & 1629 & 326 & 327 & 63 \\
\hline HG00158_h2 & 2163 & 567 & 358 & 91 \\
\hline HG00159_h1 & 1845 & 504 & 267 & 93 \\
\hline HG00159_h2 & 1422 & 351 & 339 & 83 \\
\hline HG00160_h1 & 963 & 258 & 206 & 51 \\
\hline HG00160_h2 & 1880 & 450 & 329 & 78 \\
\hline All & 1612.578125 & 421.859375 & 303.203125 & 79.328125 \\
\hline
\end{tabular}

Table 5.5: 1KG PIPE-Sites Analysis Results Summary 2 


\section{Conclusion}

While PIPE does not appear to be sensitive to SNP-induced interactome changes based solely on a change in PIPE score, PIPE was successfully used to show enrichment of SNPs at PIPE predicted interaction sites. The enrichment of SNPs in PIPE predicted interaction sites for the various datasets has two useful outcomes:

1. Validating that PIPE predicted interaction sites have some significance in relation to SNPS

2. Producing a list of interactions that are highly likely to be affected by the SNPs in question, thereby identifying biological pathways and processes affected by the SNP set

\subsection{Summary of Outcomes}

One outcome from this project is a pipeline of scripts that can easily convert SNPs into the required inputs for a PIPE run. These scripts could easily be streamlined into a web service for users to submit sets of SNPs on which PIPE could be run. SNPs from various different annotation standards, including RefSeq from the 1KG project, Uniprot from SNPdbe and PDB from SKEMPI, were converted to sequences that were run by PIPE. Conversion between these types to PIPE inputs can be done easily using scripts developed for the purpose of this project.

In addressing the first research goal from Section 1.2, PIPE was shown to perform well on the M9000 enterprise server, a medium-size compute cluster with a significant amount of shared memory. On the M9000, predictions were made, on average, every $780 \mu s$.

Also to this end, the results in Section 3, PIPE and another sequence-based 
PPI prediction method were evaluated and shown to predict little to no change on the interactome scale for single SNPs. When considering the sequence-based PIPE algorithm this result is as expected.

The second research goal was addressed in Section 4. Here it was shown that, considering the various ways in which PPI sites could be interpreted, there was statistically significant enrichment of SNPs at a subset of PIPE-predicted interaction sites. The various interpretations of PIPE-Sites included on a per-site, perinteraction and per-disease basis. These results were presented in a dynamic web graph, created using D3, and lists of significant interactions were also generated. The per-site and per-disease interpretations of PIPE-Sites were used to identify a number of interactions that are very likely affected by their corresponding SNPs. Furthermore the per-disease interpreation was used to identify diseases that may operate by disrupting PPIs as well as identify the PPIs that these diseases may disrupt.

To further illustrate the effectiveness of PIPE-Sites in relation to SNPs, a subset of disease-causing SNPs were run on PIPE. The results from this PIPE run were then analyzed and it was concluded that, though PIPE score changes are small, when a SNP occurs in a PIPE-predicted interaction site, the resulting score change is significantly higher than when the SNP occurs outside of the PIPE site. The details of this analysis can be found in Section 4.3.5.

To further understand the enrichment of SNPs at PIPE-Sites, genotypes, which are sets of known co-occurring SNPs, from the $1 \mathrm{KG}$ project were analyzed. This analysis is also the focus of the third research goal from Section 1.2. Using genotypes from 32 individuals, 2,726 unique interactions were identified that have a high probability of being affected by the genotypes of those individuals, based on a significant enrichment of SNPs within the PIPE-predicted interaction site. This 


\begin{tabular}{|c|c|c|}
\hline Outcome & Potential Applications & Supporting Chapter \\
\hline $\begin{array}{c}\text { Sequence-based PPI } \\
\text { prediction methods are not } \\
\text { sensitive to SNP-induced } \\
\text { interactome changes }\end{array}$ & $\begin{array}{l}\text { - Application of PPI pre- } \\
\text { diction methods to per- } \\
\text { sonalized medicine }\end{array}$ & 3 \\
\hline $\begin{array}{c}\text { Identify disease-related } \\
\text { SNP-induced interactome } \\
\text { changes }\end{array}$ & $\begin{array}{l}\text { - Direct research on dis- } \\
\text { ease treatment } \\
\text { - Potential drug targets }\end{array}$ & 4 \\
\hline $\begin{array}{l}\text { Identify diseases which } \\
\text { operate by PPI disruption }\end{array}$ & $\begin{array}{l}\text { - Identify disease mecha- } \\
\text { nism of action }\end{array}$ & 4 \\
\hline $\begin{array}{l}\text { Identify SNP-induced } \\
\text { interactome changes from } \\
\text { co-occurring SNPs }\end{array}$ & $\begin{array}{l}\text { - Health risk identifica- } \\
\text { tion } \\
\text { - Personalized medicine } \\
\text { - Focus for wet-lab exper- } \\
\text { iments }\end{array}$ & 5 \\
\hline
\end{tabular}

Table 6.1: Summary of Outcomes and Applications

list of interactions is publicly available from the CGM server at Carleton.

Table 6.1 summarizes the otucomes from this thesis and highlights some potential applications.

\subsection{Future Work}

A major theme for future work will be to develop a PPI prediction system capable of detecting SNP-induced changes in an individual's interactome. This requires substantially improving the sensitivity of the method, without increas- 
ing the computational complexity. This latter requirement will ensure that entire interactomes can be examined by the method within reasonable runtime.

There are various facets of analysis that could be done in the future to further the project towards its goal of providing a personalized interactome based on an individual's genome.

The mapping between types could be improved as not all mutations from SNPdbe mapped to Uniprot proteins. Mapping mutations in from the SKEMPI database to their sequences could be improved by mapping the remaining mutations that did not line up with their stated mutant amino acids.

In the SKEMPI PIPE run no proteins were removed from the interaction graph, as they were for the SNPdbe runs. This was not done in part because the PDB sequences from the SKEMPI proteins were not directly mapped to Uniprot, however it would be worth performing a sequence similarity search, such as BLAST, and then removing any of the protein interactions for which the SKEMPI sequences have a certain level of sequence identity.

Adjusting PIPE score based on a mutation being in or out of the predicted interaction site would be helpful in getting PIPE score to better reflect the effects of a mutation in an interaction site.

If PIPE were to be used for numerous personalized interactomes it would be valuable to examine certain common haplotypes, SNPs that are known to co-occur and are inherited together, and precompute the results to provide faster feedback to patients.

Besides improvements to PIPE, there is significant opportunity for improvement in the interactome and genome analysis tools available. The tool used to acquire a subset of a VCF file, vcf-subset from vcf-tools, took far longer than necessary, considering the process of computing a subset of a file is embarrass- 
ingly parallel. This tool in general could be parallelized to improve speed. As mentioned previously, a tool to convert between all annotation types would also be useful when analyzing genomic and proteomic data.

This thesis successfully shows that sequence-based tools, such as PIPE, can be used to provide some direction in predicting SNP-induced interactome changes and provides the first step towards providing personalized interactomes.

In this thesis, only SNPs were examined, which are variants that have been observed a statistically significant number of times in the population. SNVs are single nucleotide variations which may not be observed as often in the population as SNPs. SNVs represent less common variations and warrant investigation for their potential effects on the interactome. 


\section{References}

[1] A Schoenrock, F Dehne, JR Green, Ashkan Golshani, and Sylvain Pitre. MP-PIPE: a massively parallel protein-protein interaction prediction engine. Proceedings of the International Conference on Supercomputing, 2011.

[2] Bob Carlson. Next Generation Sequencing: The Next Iteration of Personalized Medicine. Biotechnology healthcare, pages 21-25, 2012.

[3] PR Bernstein, A Buschauer, and GJ Georg. Topics in Medicinal Chemistry. Springer, 2012.

[4] Christian Schaefer, Alice Meier, Burkhard Rost, and Yana Bromberg. SNPdbe: constructing an nsSNP functional impacts database. Bioinformatics (Oxford, England), 28(4):601-2, February 2012.

[5] Goncalo R Abecasis, Adam Auton, Lisa D Brooks, Mark a DePristo, Richard M Durbin, Robert E Handsaker, Hyun Min Kang, Gabor T Marth, and Gil a McVean. An integrated map of genetic variation from 1,092 human genomes. Nature, 491(7422):56-65, November 2012.

[6] Adam Amos-Binks, Catalin Patulea, Sylvain Pitre, Andrew Schoenrock, Yuan Gui, James R Green, Ashkan Golshani, and Frank Dehne. Binding site prediction for protein-protein interactions and novel motif discovery using re-occurring polypeptide sequences. BMC bioinformatics, 12:225, January 2011.

[7] Iain H Moal and Juan Fernández-Recio. SKEMPI: a Structural Kinetic and Energetic database of Mutant Protein Interactions and its use in empirical models. Bioinformatics (Oxford, England), 28(20):2600-7, October 2012. 
[8] Shawn Martin, Diana Roe, and Jean-Loup Faulon. Predicting protein-protein interactions using signature products. Bioinformatics (Oxford, England), 21(2):218-26, January 2005.

[9] Christopher M. Yates and Michael J E Sternberg. The effects of nonsynonymous single nucleotide polymorphisms (nsSNPs) on protein-protein interactions, 2013.

[10] Michael P H Stumpf, Thomas Thorne, Eric de Silva, Ronald Stewart, Hyeong Jun An, Michael Lappe, and Carsten Wiuf. Estimating the size of the human interactome. Proceedings of the National Academy of Sciences of the United States of America, 105(19):6959-6964, 2008.

[11] Arne Mu, Robert M Maccallum, and Michael J E Sternberg. Structural Characterization of the Human Proteome. Genome Research, (0):1625-1641, 2002.

[12] Jean-François Rual, Kavitha Venkatesan, Tong Hao, Tomoko HirozaneKishikawa, Amélie Dricot, Ning Li, Gabriel F Berriz, Francis D Gibbons, Matija Dreze, Nono Ayivi-Guedehoussou, Niels Klitgord, Christophe Simon, Mike Boxem, Stuart Milstein, Jennifer Rosenberg, Debra S Goldberg, Lan V Zhang, Sharyl L Wong, Giovanni Franklin, Siming Li, Joanna S Albala, Janghoo Lim, Carlene Fraughton, Estelle Llamosas, Sebiha Cevik, Camille Bex, Philippe Lamesch, Robert S Sikorski, Jean Vandenhaute, Huda Y Zoghbi, Alex Smolyar, Stephanie Bosak, Reynaldo Sequerra, Lynn DoucetteStamm, Michael E Cusick, David E Hill, Frederick P Roth, and Marc Vidal. Towards a proteome-scale map of the human protein-protein interaction network. Nature, 437(7062):1173-8, October 2005. 
[13] W Babel I Endo, S Enfors A Fiechter, M Hoare W Hu, B Mattiasson J Nielsen, K Schügerl G Stephanopoulos, U Von Stockar G T Tsao, and R Ulber C Wandrey. Computational Methods For Predicting ProteinâA $\breve{S P r o t e i n}$ Interactions. Springer-Verlag Berlin Heidleberg, 2008.

[14] Qiangfeng Cliff Zhang, Donald Petrey, Lei Deng, Li Qiang, Yu Shi, Chan Aye Thu, and Brygida Bisikirska. Structure-based prediction of protein-protein interactions on a genome-wide scale. Nature, pages 1-7, 2012.

[15] Yungki Park. Critical assessment of sequence-based protein-protein interaction prediction methods that do not require homologous protein sequences. BMC bioinformatics, 10:419, January 2009.

[16] Quan Zhong, Nicolas Simonis, Qian-Ru Li, Benoit Charloteaux, Fabien Heuze, Niels Klitgord, Stanley Tam, Haiyuan Yu, Kavitha Venkatesan, Danny Mou, Venus Swearingen, Muhammed A Yildirim, Han Yan, Amélie Dricot, David Szeto, Chenwei Lin, Tong Hao, Changyu Fan, Stuart Milstein, Denis Dupuy, Robert Brasseur, David E Hill, Michael E Cusick, and Marc Vidal. Edgetic perturbation models of human inherited disorders. Molecular Systems Biology, 5(321):321, 2009.

[17] S V Tavtigian, a M Deffenbaugh, L Yin, T Judkins, T Scholl, P B Samollow, D de Silva, a Zharkikh, and a Thomas. Comprehensive statistical study of 452 BRCA1 missense substitutions with classification of eight recurrent substitutions as neutral. Journal of medical genetics, 43(4):295-305, April 2006.

[18] Nicola S Fearnhead, Jennifer L Wilding, Bruce Winney, Susan Tonks, Sylvia Bartlett, David C Bicknell, Ian P M Tomlinson, Neil J McC Mortensen, 
and Walter F Bodmer. Multiple rare variants in different genes account for multifactorial inherited susceptibility to colorectal adenomas. Proceedings of the National Academy of Sciences of the United States of America, 101(45):15992-15997, 2004.

[19] CM Coleman and S Hannush. A novel mutation in the helix termination motif of keratin K12 in a US family with Meesmann corneal dystrophy. American Journal of Ophthalmology, pages 687-691, 1999.

[20] Richard M. Siegel, Jagan R. Muppidi, Malabika Sarker, Adrian Lobito, Melinda Jen, David Martin, Stephen E. Straus, and Michael J. Lenardo. SPOTS: Signaling protein oligomeric transduction structures are early mediators of death receptor-induced apoptosis at the plasma membrane. Journal of Cell Biology, 167(4):735-744, 2004.

[21] Hugues Abriel, Johannes Loffing, John F Rebhun, J Howard Pratt, Laurent Schild, Jean-daniel Horisberger, Daniela Rotin, and Olivier Staub. Defective regulation of the epithelial Na channel by Nedd4 in Liddles syndrome. Regulation, 103(5):667-673, 1999.

[22] William Amos. Even small SNP clusters are non-randomly distributed: is this evidence of mutational non-independence? Proceedings. Biological sciences / The Royal Society, 277(1686):1443-9, May 2010.

[23] Douglas E V Pires, David B Ascher, and Tom L Blundell. mCSM: predicting the effects of mutations in proteins using graph-based signatures. Bioinformatics (Oxford, England), 30(3):335-42, February 2014. 
[24] Prateek Kumar, Steven Henikoff, and Pauline C Ng. Predicting the effects of coding non-synonymous variants on protein function using the SIFT algorithm. Nature protocols, 4(7):1073-81, January 2009.

[25] Lei Bao and Yan Cui. Prediction of the phenotypic effects of non-synonymous single nucleotide polymorphisms using structural and evolutionary information. Bioinformatics (Oxford, England), 21(10):2185-2190, 2005.

[26] Asa Ben-Hur and William Stafford Noble. Choosing negative examples for the prediction of protein-protein interactions. BMC bioinformatics, $7 \mathrm{Suppl}$ 1:S2, January 2006.

[27] ACR Martin. Mapping PDB chains to UniProtKB entries. Bioinformatics, $00(00): 1-6,2005$.

[28] Yungki Park and Edward M Marcotte. Revisiting the negative example sampling problem for predicting protein-protein interactions. Bioinformatics (Oxford, England), 27(21):3024-8, November 2011.

[29] John D. Storey. A direct approach to false discovery rates. Journal of the Royal Statistical Society: Series B (Statistical Methodology), 64(3):479-498, August 2002.

[30] John D Storey and Robert Tibshirani. Statistical significance for genomewide studies. Proceedings of the National Academy of Sciences of the United States of America, 100(16):9440-5, August 2003.

[31] Michael Bostock, Vadim Ogievetsky, and Jeffrey Heer. D3: Data-Driven Documents. IEEE transactions on visualization and computer graphics, 17(12):2301-9, December 2011. 
[32] Franziska M Gisler, Thomas von Kanel, Richard Kraemer, André Schaller, and Sabina Gallati. Identification of SNPs in the cystic fibrosis interactome influencing pulmonary progression in cystic fibrosis. European journal of human genetics : EJHG, 21(4):397-403, April 2013.

[33] Venüs Ummiye Onay, Laurent Briollais, Julia a Knight, Ellen Shi, Yuanyuan Wang, Sean Wells, Hong Li, Isaac Rajendram, Irene L Andrulis, and Hilmi Ozcelik. SNP-SNP interactions in breast cancer susceptibility. BMC cancer, 6:114, January 2006.

[34] Gene Herg Mutations, Eckhard Ficker, Carlos A Obejero-paz, Shuxia Zhao, and Arthur M Brown. The Binding Site for Channel Blockers That Rescue Misprocessed Human Long QT Syndrome Type 2 ether-a-gogo-related Gene (HERG) Mutations. Journal of Biological Chemistry, 2002.

[35] Heng Li, Bob Handsaker, Alec Wysoker, Tim Fennell, Jue Ruan, Nils Homer, Gabor Marth, Goncalo Abecasis, and Richard Durbin. The Sequence Alignment/Map format and SAMtools. Bioinformatics (Oxford, England), 25(16):2078-9, August 2009.

[36] Kai Wang, Mingyao Li, and Hakon Hakonarson. ANNOVAR: functional annotation of genetic variants from high-throughput sequencing data. Nucleic acids research, 38(16):e164, September 2010. 


\section{A Pseudo Code, Extraneous Data}

\section{A.1 Optimization of the PIPE Algorithm}

In order to run interactome-wide PPI comparisons, the PIPE algorithm was parallelized and optimized in a number of ways. There were speed improvements to facilitate running the algorithm in a virtual environment as well as parallelization which enabled scaling of the PIPE algorithm to different architectures. These optimizations facilitate PIPE's ability to make fast, interactome-wide PPI predictions.

In the Uniprot protein database used for experiments there are 20,273 proteins and 78,346 known interactions for humans. A full all-to-all run of the PIPE algorithm, making predictions on every possible pair of proteins, totals over 410 million predictions. Since the PIPE algorithm sequentially analyzes small subsequences of a protein, longer proteins take longer to run. The protein neighbour list, which directly affects run-time, in the human protein set is highly variable, which can lead to high latency if the algorithm is not properly load balanced.

Three critical improvements were made to the sequential algorithm that make it possible to perform interactome-wide comparisons in a reasonable amount of time. The first of these is storing the protein sequence as a binary number instead of a sequence of characters. This significantly decreases the space needed for each protein sequence and removed the need to convert a character to an index when calculating PAM scores.

The second improvement to the sequential algorithm applies incremental updates to the sliding window methodology, moving both windows together in sync. For two proteins with $m$ and $n$ windows to be compared, the number of addition/subtraction operations to perform the exhaustive comparison of all fragmetns 
is decreased from $n \times m$ to $\frac{n \times m}{2}$ by applying incremental updates.

The third improvement used to provide significant speedup was the creation of a database of the interaction partners for all possible sequences. When the list of neighbours, $R$, is being constructed this means that a lookup operation is performed in the database as opposed to performing an exhaustive search through the interaction graph itself. These three techniques provide significant speed gains to the sequential PIPE algorithm[1].

The algorithm described in Section 2.5.1 requires no interaction between each individual PIPE run and as a result making predictions for large numbers of interactions is an embarrasingly parallel problem. For this reason PIPE is designed to be run on a number of parallel architectures from large scale commodity clusters to smaller scale coarse grained multicomputers. There are a number of levels of parallelism possible for the PIPE algorithm, however a two tiered master-slave architecture was used to make PIPE more flexible and portable[1].

One of the main challenges in developing an efficient parallel implementaiton of the PIPE algorithm is the highly variable run-time for each protein pair prediction. There are two main cases that can cause increased runtime. In the first case, if during the fragment search in protein $A$, fragment $a_{i}$ has a large number of similar sequences and the resulting neighbour set, $R$, is large, then all fragments must be compared with all these neighbours in the exhaustive search.

The second factor that leads to a highly variable runtime is the protein length itself. In the human dataset there are proteins as small as 4 amino acids in length and some proteins that are in excess of 34000 amino acids long. The change in length significantly changes the number of comparison/lookup operations that must be performed because a longer protein has more fragments to compare. These two factors make load balancing in the parallel implementation of PIPE of 
high importance for fast and efficient execution.

The two tiered master-slave architecture has a single node acting as the scheduler which divides the input pairs into packets, distributes these packets to the worker nodes then compiles the results as they are returned from the PIPE worker threads. When a node completes predictions on the pairs in a packet, it is assigned more work if there are still packets remaining at the scheduler on which predictions need to be made. The packet size cannot be too small as this will lead to excess communication, however if the packet size is too large there can be large work imbalances. The master scheduler algorithm is shown in Algorithm 2 and is implemented in MPI.

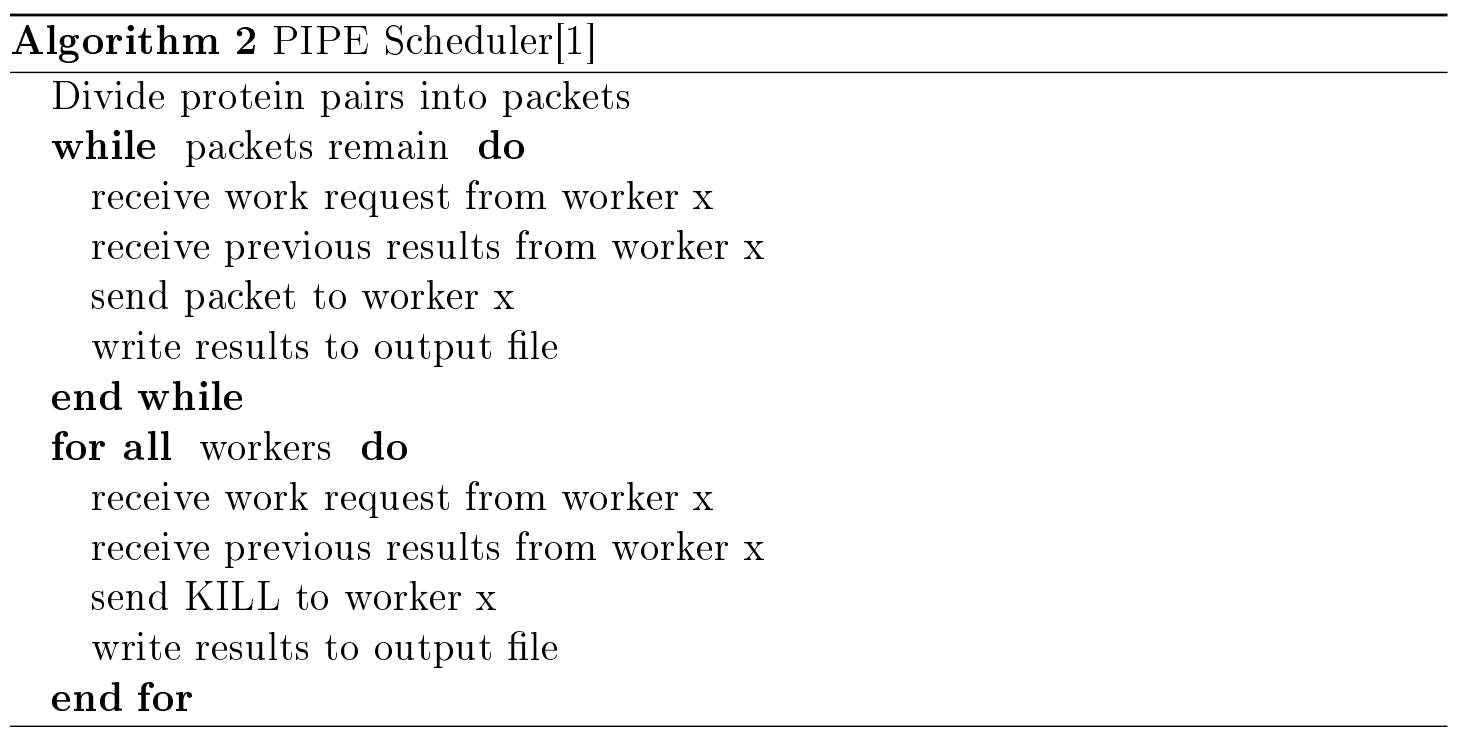

The PIPE worker begins by initializing the interaction graph, $G$, and the database of protein fragments. The PIPE worker then launches several worker threads to begin running the PIPE algorithm on the pairs received from the master scheduler. If there are no longer any pairs to be operated on in the current packet, the first worker thread to have no more input pairs to work on sends the results to the scheduler then requests another packet to perform work on. If the message 
received is the KILL signal, all processes exit. Each worker thread shares a copy of the PIPE interaction graph as well as the fragment database discussed previously. The results file, interaction graph and database are all parallel data structures that can be operated on concurrently by all worker threads. The PIPE worker code is shown in Algorithm 3 and is implemented in OpenMP.

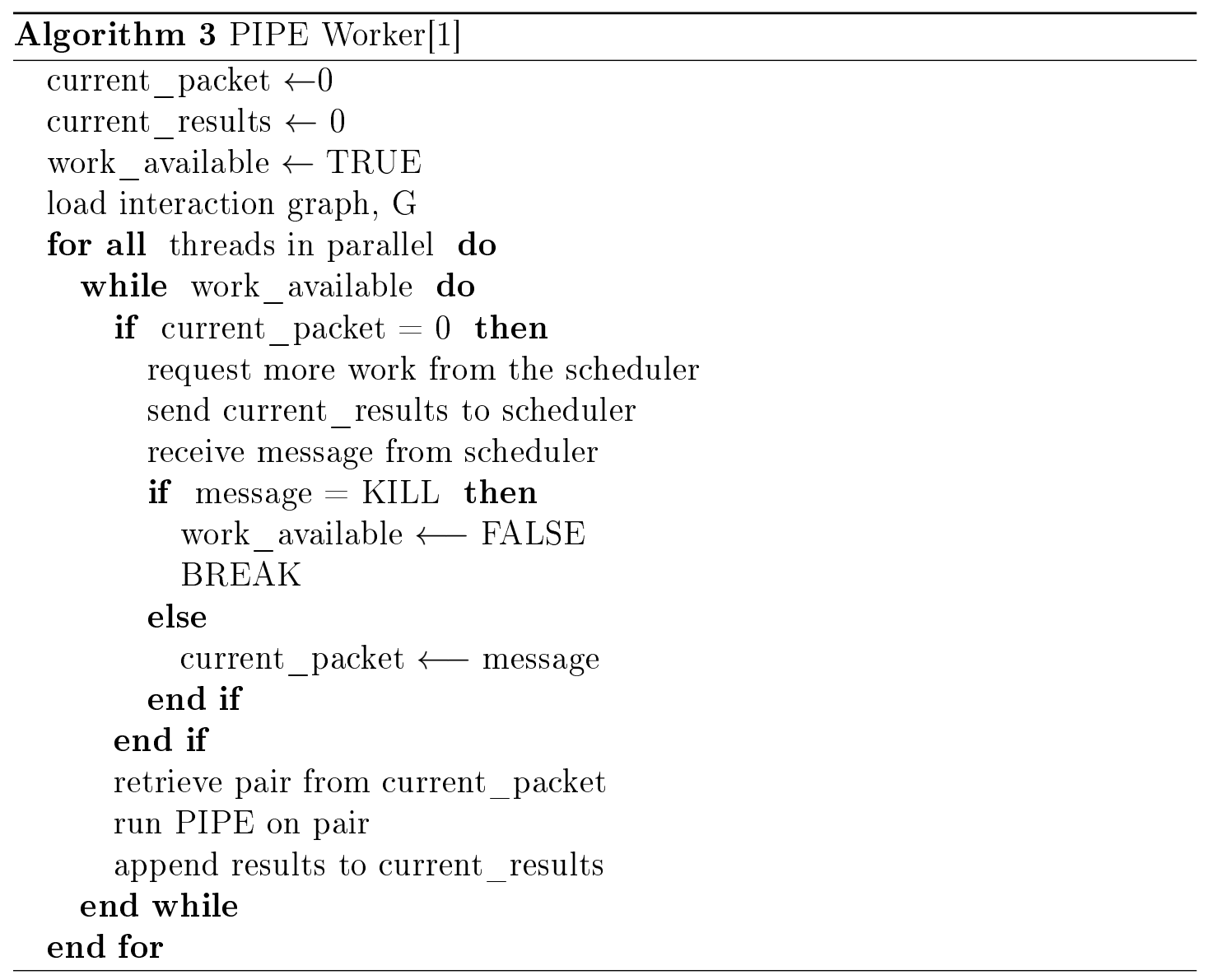

The number of PIPE workers is specified at run time and then number of threads is hardcoded into the PIPE code itself. Figure A.1 shows an overview of the architecture of the tiered master-slave architecture of PIPE. 


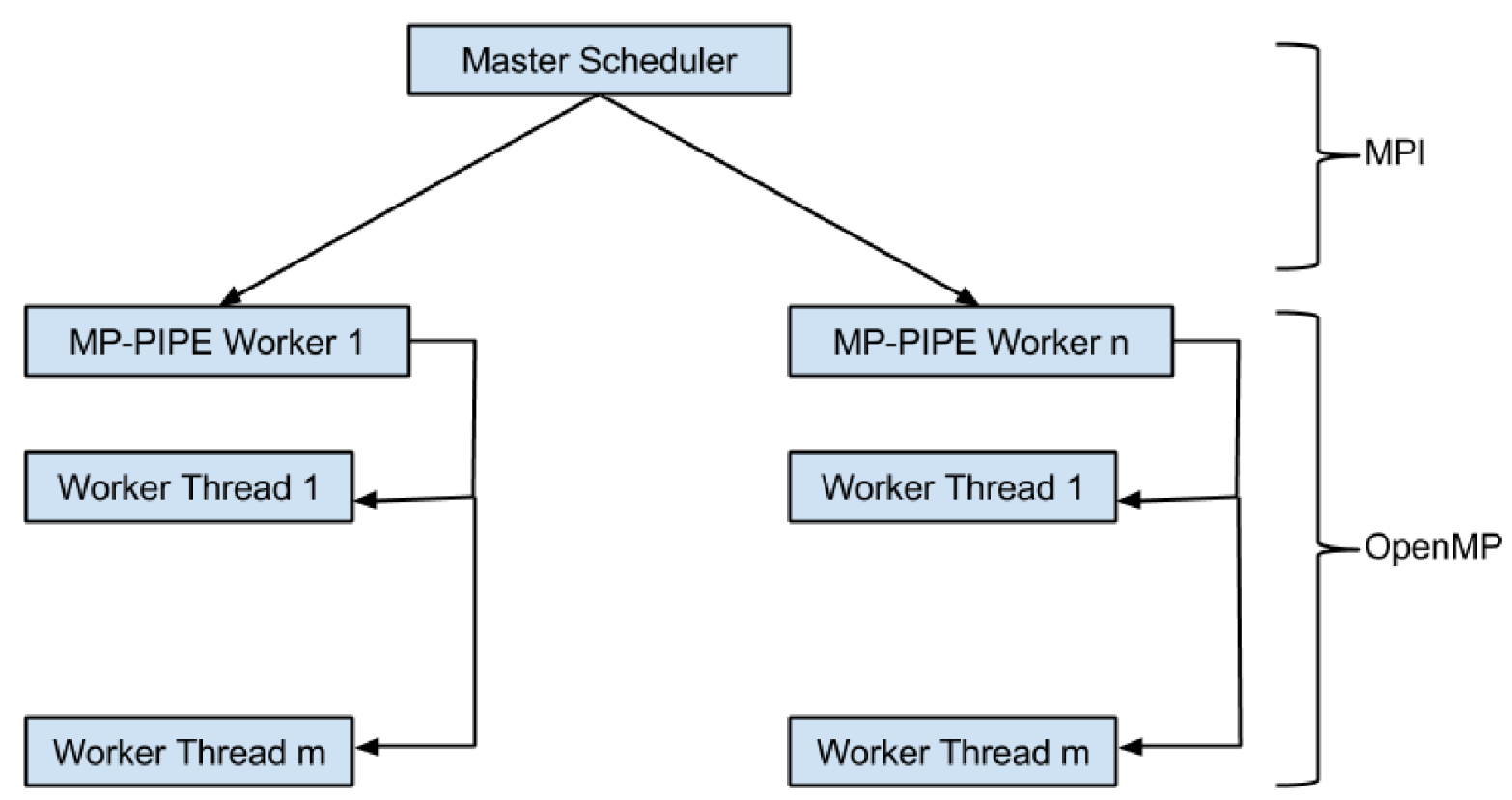

Figure A.1: PIPE Overview

\section{A.2 Pseudo-Code for Input Generation}

The pseudo-code in Algorithm 4 summarizes the process of creating the input protein pairs for each separate SNP file.

The code initializes all variables at the beginning, including input_proteins, which are the proteins that require a one-to-all input pair file, the wild_type_proteins set which is the set of only the wild type proteins.

The first loop goes through the sequence file containing the SNPs and adds the SNP names to the input_proteins array.

The second loop goes through the wild type sequence file and creates a set of wild type proteins used to generate the input pairs for the one to all run. The wild type proteins are then added to the list of proteins that need input one-to-all files created.

The third loop goes through each protein needing a one-to-all file and creates 


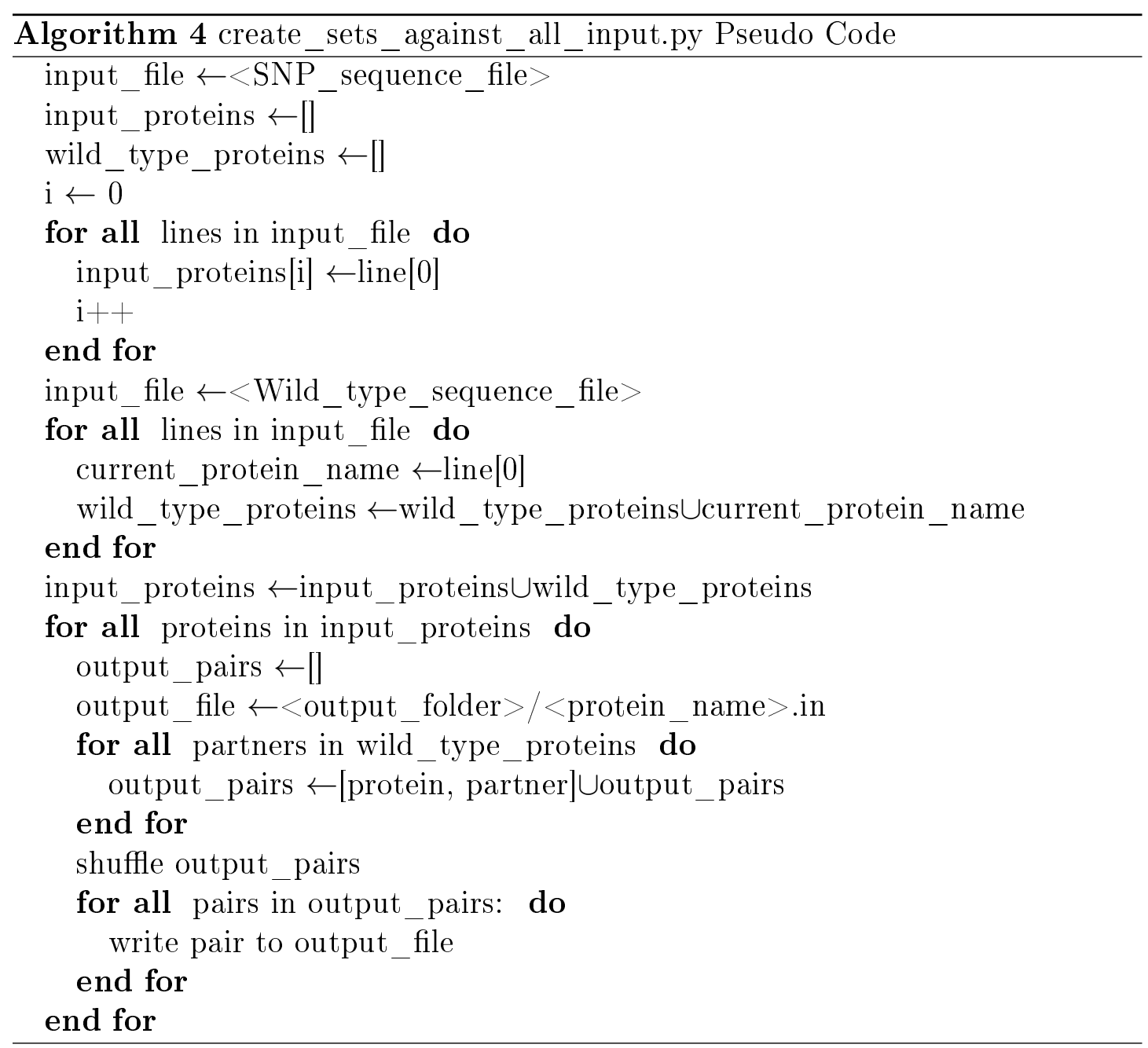


the file named after the protein, whether it is a wild type or mutated protein.

This loop then creates an array of input protein pairs using the current protein and all other wild type proteins from the wild type protein set created previously. The output pairs are then shuffled and written to the file.

When run, the script will generate pruned interaction graph files using the file names in the input pair files folder. Algorithm 5 describes the code used to generate the interaction graph files.

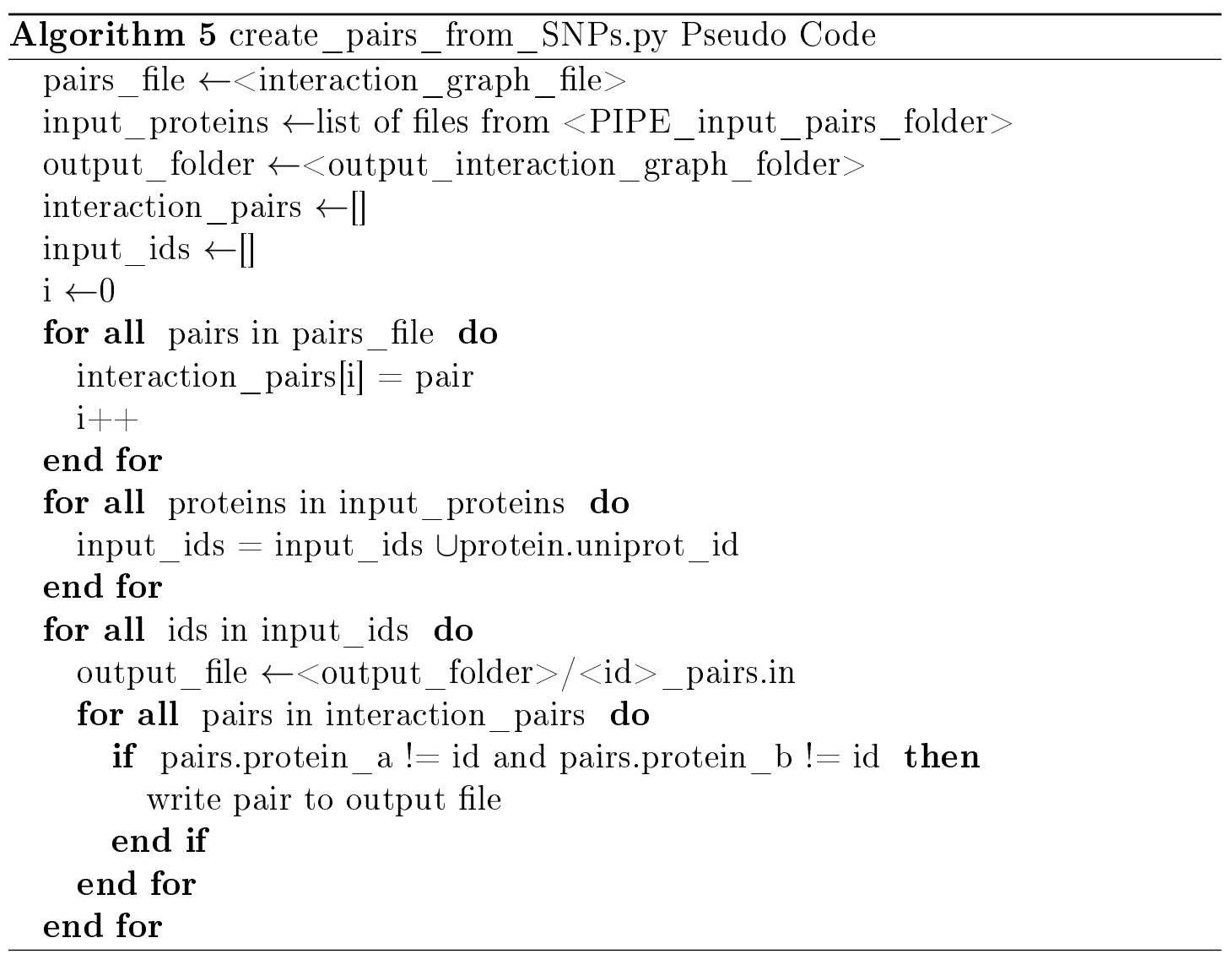

All variables are initialized at the beginning of the code shown in Algorithm 5. The interaction grap file is loaded into pairs_file, input_proteins is initialized to be the list of files from the input pairs directory, the output folder is taken directly from the command line argument and the other variables are initialized to null 
or zero values. The interaction pairs file will hold each pair of known interacting proteins, input_ids is a set of all the wild type Uniprot IDs and i is used as the index for populating the interaction_pairs array.

The first for loop populates the interaction_pairs array with the known interactions pairs from the interaction graph.

The second for loop populates the input_ids set with the uniprot ID of all the proteins involved in the SNPs.

The third for loop iterates through the Uniprot IDs in the input_ids set and creates the customized interaction graph for that protein. Each interaction pair is then examined and the pairs that do not contain the current protein are written to the pruned interaction graph.

The pseudo code for the script is shown in Algorithm 6 .

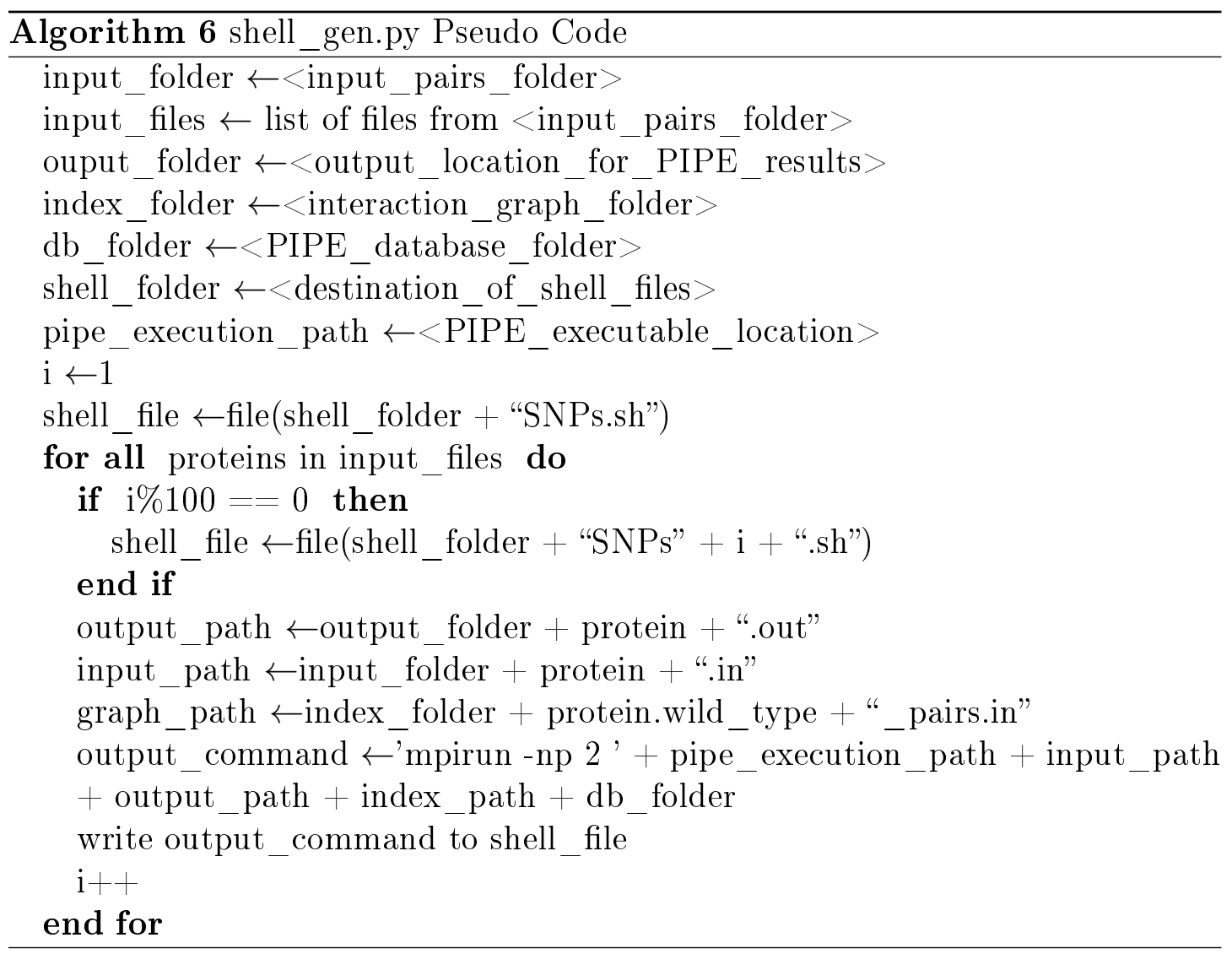


The initial commands in Algorithm 6 prepare each of the file paths needed for the PIPE run. The input folder is the location of the input pairs, that is the one-to-all runs for each SNP and wild type proteins and input_files is the list of all files from this directory.

The output folder is the location of the PIPE output, not the output for the shell scripts.

The index folder is the location of the interaction graph files, db_folder is the location of the PIPE window database, the shell folder is where the shell scripts generated will be output and the pipe_execution_path is the location of the PIPE executable used to execute PIPE.

Once all these parameters are initialized all the one-to-all input files are looped over by the for loop. For each mutated or wild type protein, the PIPE run command line information described previously is generated and appended to the shell script file. Once a file reaches 100 PIPE run commands it is closed and a new file started. Each shell script is named using the convention: SNPS $<$ index_of_first_SNP_run $>$. Using this convention the first shell script is SNPs.sh, the second which starts with the 101st SNP one-to-all run is SNPs100.sh and so on.

\section{A.3 Generating Interaction Graphs for Individual PIPE Runs}

After generating the input pairs for each SNP and each wild type protein, the pruned interaction graph files must similarly be generated. This process involves removing the known interactions for the wild-type protein in which a SNP occurs and creating an interaction graph specific to the mutated protein. The required inputs for this script are:

1. Folder location of the PIPE input pair files 


\begin{tabular}{|c|c|c|c|}
\hline Interaction Protein & Mutant Score & Wild Type Score & Difference in PIPE Score \\
\hline \hline Q9Y4D1 & 0.015 & 0.016 & 0.001 \\
\hline
\end{tabular}

Table A.1: Example PIPE Analysis File of a SNP

2. Original interaction graph file

3. Output folder for pruned interaction graph files

This script has the following usage:

python create_pairs_from_SNPs.py <input1><input2><input3>

Where input1 is the folder where the input pairs for PIPE are located, input2 is the original interaction graph and input3 is the destination folder for all the interaction graphs files.

It is important to note that the input pairs must have the naming convention discussed previously and all files must be tab delimited.

\section{A.4 SKEMPI PIPE Run Analysis Files}

As discussed, since there were no interactions pruned from the interaction graph for the SKEMPI protein sequences, each sequence did not need a unique interaction graph. Since all test pairs shared the same interaction graph, there was no need to separate the input pairs into separate files either. This resulted in a single PIPE run for all the test pairs for all SKEMPI proteins, totalling 65,300,873 as mentioned in Section 3.1.

Once these analysis files were created, the results were analyzed in a number of different ways.

When the decision threshold is applied to the mutated PIPE scores, in total there was only one gained interaction and no lost interactions. The gained 
interaction occurred between the mutant protein Q8NCB2_R39W and Q156A1. Originally the wild type interaction between Q156A1 and Q8NCB2 was right on the edge of being a predicted interaction with a PIPE score of 0.90510850 . The change from a an Arginine(R) to a Tryptophan(W) cause the slight increase in PIPE score needed to predict the mutated pairs to interact.

\section{A.5 Per Protein Interaction Site Analysis of SNP Locations}

Extending the idea of looking at the coverage of a PIPE site, the next variation on PIPE site analysis looked at the union of all PIPE sites in a protein from all positive interactions.

The value for $\mathrm{p}$ in Equation 4.2 in this case is taken to be the proportion of the protein in which the mutations occurs that is covered by the PIPE site in question. For a protein $x$ this can be calculated using the formula:

$$
p=\frac{\mid \text { site }_{\text {total }} \mid}{|x|}
$$

Where the magnitude on the bottom is the length of protein $x$. The site $_{\text {total }}$ value is calculated using all the edges, E, or interactions, for the protein $x$ that were predicted using the PIPE algorithm. This value is calculated as follows.

$$
\forall e \in E_{x}: \forall \text { site } \in e: \text { site }_{\text {total }} \leftarrow \text { site }_{\text {total }} \cup \text { site }
$$

Equation A.2 states that for each interaction in the positive interactions for protein $x$, site total $_{\text {is }}$ updated to be union of all PIPE interactions sites in that interaction.

The number of positive occurrences, $k$ from Equation 4.2, is calculated as the 


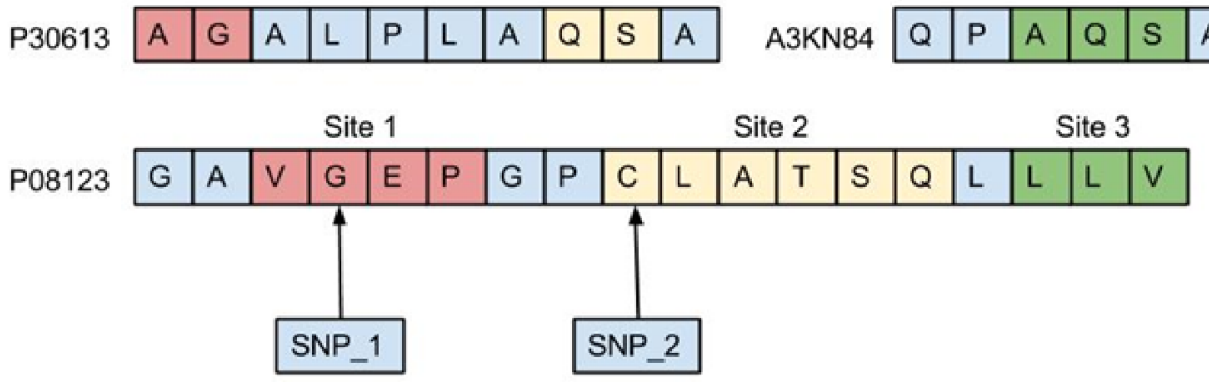

Figure A.2: Per Protein Interaction P-Value Analysis

number of SNPs that occur in that protein that fall into the site $_{\text {total }}$ amalgamated site.

The population size, $n$ from Equation 4.2, is calculated as the number of SNPs from the SNPdbe database, that occur in the protein in question.

Figure A.2 shows the PIPE sites from two interacting proteins. The coverage of the PIPE site is the total coverage for both sets of PIPE sites and in total is $\frac{13}{18}$.

\section{A.5.1 Per Protein Results}

The Per Protein analysis results once again only looked at proteins with more than 50 SNPs to minimize the probability of randomness affecting results. In total there were 111 proteins with more than 50 SNPs. Of these 111 proteins, 13 had a p-value of significance at a cutoff of 0.05 . The rest of the results were relatively uniformly spread between 0 and 1 . 


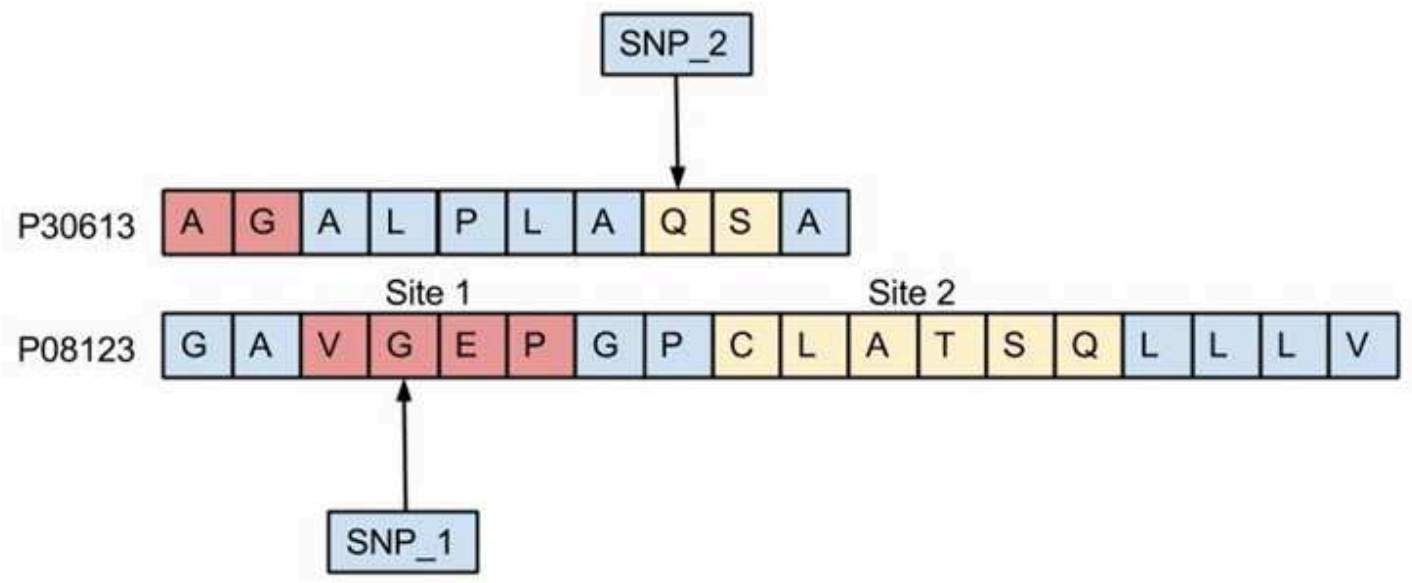

Figure A.3: Per Interaction P-Value Analysis

\section{A.6 Per Interaction, Interaction Site Analysis of SNP Lo- cations}

The next analysis of the location of SNPs in relation to PIPE sites extended the idea of the per protein/per interaction analysis and analyzed each interaction as a separate binomial distribution. In this analysis, only proteins which had more than 50 SNPs combined between the two proteins interacting were used. Figure A.3 shows an example of a protein interaction used in the per interaction analysis.

In Figure A.3, there are two SNPs, one in each of the proteins. The analysis in this case calculates the coverage of the PIPE site, $\mathrm{p}$ from Equation 4.2, is the length of the union of the PIPE sites in both proteins divided by the length of both proteins. For two proteins, x and y, Equation A.3 would be used to calculate the coverage.

$$
p=\frac{\mid \text { site }_{1 x} \cup \text { site }_{2 x} \cup \text { site }_{3 x} \cup \text { site }_{1 y} \cup \text { site }_{1 y} \cup \text { site }_{1 y} \mid}{|x|+|y|}
$$

Furthermore, $k$ is the number of SNPs that occur in both proteins and $n$ is 


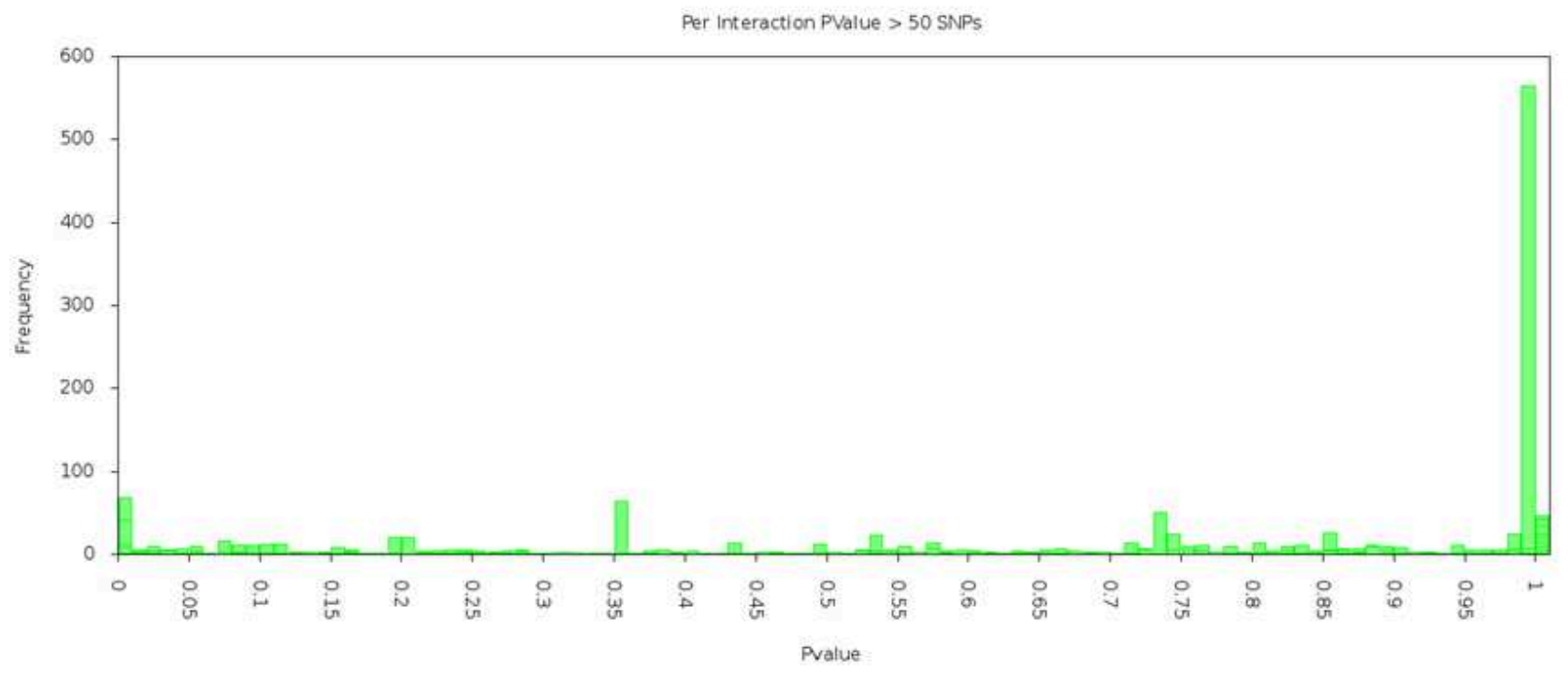

Figure A.4: Per Interaction Analysis Histogram of P-values

the total number of SNPs that occur in both proteins.

\section{A.6.1 Per Interaction Analysis Results}

In all there were 4,648 interactions analyzed, 24 more than the per protein/per interaction analysis. This addition was because of the inclusion of the SNPS to the interaction partner of each protein in the interaction. Of these 4,648 , 531 had statistically significant p-value at a significance of 0.05 . This marks an increase of 194 interactions compared with the per protein/per interaction analysis, which is not unexpected as the coverage of the PIPE site was decreased in most cases, resulting in a lower probability of a positive occurence, the value $p$, from Equation4.2. Figure A.4 shows the histogram of the p-values for all the interactions analysed.

It should be noted that the peak for the per interaction analysis had a much higher peak around 1 than the per protein/per interaction analysis, though it also had more significant interactions. 


\section{A.7 Significant Interactions at a Q-Value of 0.001}

\begin{tabular}{|c|c|c|c|}
\hline disease & $\mathrm{p} 1$ & $\mathrm{p} 2$ & num_mutations \\
\hline \hline long qt syndrome type 2 & Q12809 & Q517T1 & 67 \\
\hline liddle syndrome & P51168 & Q9BXS5 & 5 \\
\hline liddle syndrome & P51168 & Q8N668 & 5 \\
\hline liddle syndrome & P51168 & Q9Y6Q5 & 5 \\
\hline $\begin{array}{c}\text { cerebral autosomal dominant } \\
\text { arteriopathy with subcortical }\end{array}$ & Q9UM47 & O00548 & 108 \\
\hline $\begin{array}{c}\text { leukoencephalopathy } \\
\text { cerebral autosomal dominant } \\
\text { arteriopathy with subcortical }\end{array}$ & Q9UM47 & Q7Z3S9 & 108 \\
\hline $\begin{array}{c}\text { leukoencephalopathy } \\
\text { renal cell carcinoma papillary }\end{array}$ & P08581 & Q07890 & 15 \\
\hline renal cell carcinoma papillary & P08581 & O95757 & 15 \\
\hline renal cell carcinoma papillary & P08581 & O15357 & 15 \\
\hline renal cell carcinoma papillary & P08581 & P14210 & 15 \\
\hline renal cell carcinoma papillary & P08581 & Q07889 & 15 \\
\hline renal cell carcinoma papillary & P08581 & P22681 & 15 \\
\hline renal cell carcinoma papillary & P08581 & P40763 & 15 \\
\hline renal cell carcinoma papillary & P08581 & Q6PJ21 & 15 \\
\hline renal cell carcinoma papillary & P08581 & Q99619 & 15 \\
\hline
\end{tabular}




\begin{tabular}{|c|c|c|c|}
\hline $\begin{array}{c}\text { amyloidosis } \\
\text { transthyretin-related }\end{array}$ & $\mathrm{P} 02766$ & Q9BQE3 & 73 \\
\hline cancer & $\mathrm{P} 42336$ & $\mathrm{P} 07948$ & 27 \\
\hline cancer & $\mathrm{P} 42336$ & $\mathrm{P} 06239$ & 27 \\
\hline $\begin{array}{c}\text { myelogenous leukemia } \\
\text { lymphoblastic leukemia } \\
\text { patients and in acute } \\
\text { myelogenous leukemia } \\
\text { lymphoblastic leukemia }\end{array}$ & $\mathrm{P} 36888$ & $\mathrm{O} 43255$ & 5 \\
\hline $\begin{array}{c}\text { myelogenous leukemia } \\
\text { lymphoblastic leukemia } \\
\text { patients and in acute } \\
\text { myelogenous leukemia } \\
\text { lymphoblastic leukemia }\end{array}$ & $\mathrm{P} 36888$ & $\mathrm{O} 15524$ & 5 \\
\hline $\begin{array}{c}\text { myelogenous leukemia } \\
\text { lymphoblastic leukemia } \\
\text { patients and in acute } \\
\text { myelogenous leukemia } \\
\text { lymphoblastic leukemia }\end{array}$ & $\mathrm{P} 36888$ & $\mathrm{P} 08238$ & 5 \\
\hline $\begin{array}{c}\text { myelogenous leukemia } \\
\text { lymphoblastic leukemia } \\
\text { patients and in acute } \\
\text { myelogenous leukemia } \\
\text { lymphoblastic leukemia }\end{array}$ & $\mathrm{P} 36888$ & O14933 & \\
\hline & & & \\
\hline
\end{tabular}




\begin{tabular}{|c|c|c|c|}
\hline $\begin{array}{c}\text { myelogenous leukemia } \\
\text { lymphoblastic leukemia } \\
\text { patients and in acute } \\
\text { myelogenous leukemia } \\
\text { lymphoblastic leukemia }\end{array}$ & P36888 & Q13588 & 5 \\
\hline $\begin{array}{c}\text { myelogenous leukemia } \\
\text { lymphoblastic leukemia } \\
\text { patients and in acute } \\
\text { myelogenous leukemia } \\
\text { lymphoblastic leukemia }\end{array}$ & P36888 & P07900 & 5 \\
\hline $\begin{array}{c}\text { myelogenous leukemia } \\
\text { lymphoblastic leukemia } \\
\text { patients and in acute } \\
\text { myelogenous leukemia } \\
\text { lymphoblastic leukemia }\end{array}$ & P36888 & P0CG48 & \\
\hline $\begin{array}{c}\text { myelogenous leukemia } \\
\text { lymphoblastic leukemia } \\
\text { patients and in acute } \\
\text { myelogenous leukemia } \\
\text { lymphoblastic leukemia }\end{array}$ & P36888 & Q156A1 & 5 \\
\hline $\begin{array}{c}\text { myelogenous leukemia } \\
\text { lymphoblastic leukemia } \\
\text { patients and in acute } \\
\text { myelogenous leukemia } \\
\text { lymphoblastic leukemia }\end{array}$ & P36888 & P0CG47 & \\
\hline & & & \\
\hline
\end{tabular}




\begin{tabular}{|c|c|c|c|}
\hline $\begin{array}{c}\text { myelogenous leukemia } \\
\text { lymphoblastic leukemia } \\
\text { patients and in acute } \\
\text { myelogenous leukemia } \\
\text { lymphoblastic leukemia }\end{array}$ & P36888 & Q8IUQ4 & 5 \\
\hline $\begin{array}{c}\text { myelogenous leukemia } \\
\text { lymphoblastic leukemia } \\
\text { patients and in acute } \\
\text { myelogenous leukemia } \\
\text { lymphoblastic leukemia }\end{array}$ & P36888 & Q58FF6 & 5 \\
\hline $\begin{array}{c}\text { myelogenous leukemia } \\
\text { lymphoblastic leukemia } \\
\text { patients and in acute } \\
\text { myelogenous leukemia } \\
\text { lymphoblastic leukemia }\end{array}$ & P36888 & Q92569 & \\
\hline $\begin{array}{c}\text { myelogenous leukemia } \\
\text { lymphoblastic leukemia } \\
\text { patients and in acute } \\
\text { myelogenous leukemia } \\
\text { lymphoblastic leukemia }\end{array}$ & P36888 & Q15843 & 5 \\
\hline $\begin{array}{c}\text { myelogenous leukemia } \\
\text { lymphoblastic leukemia } \\
\text { patients and in acute } \\
\text { myelogenous leukemia } \\
\text { lymphoblastic leukemia }\end{array}$ & P36888 & Q8IXJ6 & \\
\hline & & & \\
\hline
\end{tabular}




\begin{tabular}{|c|c|c|c|}
\hline $\begin{array}{c}\text { myelogenous leukemia } \\
\text { lymphoblastic leukemia } \\
\text { patients and in acute } \\
\text { myelogenous leukemia } \\
\text { lymphoblastic leukemia }\end{array}$ & P36888 & P27986 & 5 \\
\hline $\begin{array}{c}\text { myelogenous leukemia } \\
\text { lymphoblastic leukemia } \\
\text { patients and in acute } \\
\text { myelogenous leukemia } \\
\text { lymphoblastic leukemia }\end{array}$ & P36888 & Q58FF8 & 5 \\
\hline $\begin{array}{c}\text { myelogenous leukemia } \\
\text { lymphoblastic leukemia } \\
\text { patients and in acute } \\
\text { myelogenous leukemia } \\
\text { lymphoblastic leukemia }\end{array}$ & P36888 & Q9Y6K9 & 5 \\
\hline $\begin{array}{c}\text { myelogenous leukemia } \\
\text { lymphoblastic leukemia } \\
\text { patients and in acute } \\
\text { myelogenous leukemia } \\
\text { lymphoblastic leukemia }\end{array}$ & P36888 & Q58FG1 & \\
\hline $\begin{array}{c}\text { myelogenous leukemia } \\
\text { lymphoblastic leukemia } \\
\text { patients and in acute } \\
\text { myelogenous leukemia } \\
\text { lymphoblastic leukemia }\end{array}$ & P36888 & P62993 & 5 \\
\hline
\end{tabular}




\begin{tabular}{|c|c|c|c|}
\hline $\begin{array}{c}\text { myelogenous leukemia } \\
\text { lymphoblastic leukemia } \\
\text { patients and in acute } \\
\text { myelogenous leukemia } \\
\text { lymphoblastic leukemia }\end{array}$ & P36888 & Q14568 & 5 \\
\hline $\begin{array}{c}\text { myelogenous leukemia } \\
\text { lymphoblastic leukemia } \\
\text { patients and in acute } \\
\text { myelogenous leukemia } \\
\text { lymphoblastic leukemia }\end{array}$ & P36888 & Q58FG0 & 5 \\
\hline $\begin{array}{c}\text { myelogenous leukemia } \\
\text { lymphoblastic leukemia } \\
\text { patients and in acute } \\
\text { myelogenous leukemia } \\
\text { lymphoblastic leukemia }\end{array}$ & P36888 & Q58FF7 & 5 \\
\hline meesmann corneal dystrophy & Q99456 & P27348 & 12 \\
\hline In colorectal adenomas & P01116 & O75398 & 8 \\
\hline In colorectal adenomas & P01116 & P04637 & 8 \\
\hline In colorectal adenomas & $\mathrm{P} 01116$ & O15379 & 8 \\
\hline In colorectal adenomas & P01116 & P0CG47 & 8 \\
\hline In colorectal adenomas & $\mathrm{P} 01116$ & Q7Z569 & 8 \\
\hline In colorectal adenomas & P01116 & Q99832 & 8 \\
\hline In colorectal adenomas & P01116 & Q07889 & 8 \\
\hline In colorectal adenomas & P01116 & O14827 & 8 \\
\hline
\end{tabular}




\begin{tabular}{|c|c|c|c|}
\hline In colorectal adenomas & P01116 & Q9BY41 & 8 \\
\hline In colorectal adenomas & P01116 & Q15843 & 8 \\
\hline In colorectal adenomas & P01116 & Q96B97 & 8 \\
\hline In colorectal adenomas & P01116 & P04049 & 8 \\
\hline In colorectal adenomas & P01116 & P27986 & 8 \\
\hline In colorectal adenomas & P01116 & Q13009 & 8 \\
\hline In colorectal adenomas & P01116 & Q05639 & 8 \\
\hline In colorectal adenomas & P01116 & Q13574 & 8 \\
\hline In colorectal adenomas & P01116 & P50749 & 8 \\
\hline In colorectal adenomas & P01116 & P10398 & 8 \\
\hline In colorectal adenomas & P01116 & O60812 & 8 \\
\hline In colorectal adenomas & P01116 & Q9UQ13 & 8 \\
\hline In colorectal adenomas & P01116 & Q9Y6X8 & 8 \\
\hline In colorectal adenomas & P01116 & P55196 & 8 \\
\hline In colorectal adenomas & P01116 & P06213 & 8 \\
\hline In colorectal adenomas & P01116 & Q8WWW0 & 8 \\
\hline In colorectal adenomas & P01116 & P16452 & 8 \\
\hline In colorectal adenomas & P01116 & Q5VU43 & 8 \\
\hline In colorectal adenomas & P01116 & Q156A1 & 8 \\
\hline In colorectal adenomas & P01116 & Q96RT1 & 8 \\
\hline In colorectal adenomas & P01116 & Q92769 & 8 \\
\hline In colorectal adenomas & P01116 & Q13972 & 8 \\
\hline In colorectal adenomas & P01116 & P07910 & 8 \\
\hline In colorectal adenomas & P01116 & P0CG48 & 8 \\
\hline
\end{tabular}




\begin{tabular}{|c|c|c|c|}
\hline In colorectal adenomas & P01116 & P60866 & 8 \\
\hline In colorectal adenomas & P01116 & Q96ID5 & 8 \\
\hline In colorectal adenomas & P01116 & Q96EY1 & 8 \\
\hline In colorectal adenomas & P01116 & Р00749 & 8 \\
\hline In colorectal adenomas & P01116 & Q9P212 & 8 \\
\hline In colorectal adenomas & P01116 & P52306 & 8 \\
\hline In colorectal adenomas & P01116 & P22681 & 8 \\
\hline In colorectal adenomas & P01116 & P01112 & 8 \\
\hline In colorectal adenomas & P01116 & Q92888 & 8 \\
\hline In colorectal adenomas & P01116 & Q15717 & 8 \\
\hline In colorectal adenomas & P01116 & Q12967 & 8 \\
\hline In colorectal adenomas & P01116 & Q9NS23 & 8 \\
\hline In colorectal adenomas & P01116 & P01111 & 8 \\
\hline In colorectal adenomas & P01116 & Q13671 & 8 \\
\hline In colorectal adenomas & P01116 & P21359 & 8 \\
\hline In colorectal adenomas & P01116 & Q9NXA8 & 8 \\
\hline In colorectal adenomas & P01116 & P27482 & 8 \\
\hline In colorectal adenomas & P01116 & Q8IZJ4 & 8 \\
\hline In colorectal adenomas & P01116 & Q9UJ41 & 8 \\
\hline In colorectal adenomas & P01116 & P81274 & 8 \\
\hline In colorectal adenomas & P01116 & P25685 & 8 \\
\hline In colorectal adenomas & P01116 & Q96KQ4 & 8 \\
\hline In colorectal adenomas & P01116 & Q9NRM7 & 8 \\
\hline In colorectal adenomas & P01116 & Q5U651 & 8 \\
\hline
\end{tabular}




\begin{tabular}{|c|c|c|c|}
\hline In colorectal adenomas & P01116 & P15056 & 8 \\
\hline In colorectal adenomas & P01116 & Q02750 & 8 \\
\hline In colorectal adenomas & P01116 & Q99614 & 8 \\
\hline In colorectal adenomas & P01116 & A6NIZ1 & 8 \\
\hline In colorectal adenomas & P01116 & O00329 & 8 \\
\hline In colorectal adenomas & P01116 & O43924 & 8 \\
\hline In colorectal adenomas & P01116 & P48736 & 8 \\
\hline In colorectal adenomas & P01116 & B7ZW38 & 8 \\
\hline Antithrombin III deficiency & P01008 & Q15843 & 16 \\
\hline Antithrombin III deficiency & P01008 & P0CG48 & 16 \\
\hline Antithrombin III deficiency & P01008 & P0CG47 & 16 \\
\hline $\begin{array}{c}\text { pachyonychia congenita type } \\
2\end{array}$ & Q04695 & P08670 & 9 \\
\hline $\begin{array}{l}\text { pachyonychia congenita type } \\
2\end{array}$ & Q04695 & $\mathrm{P} 08727$ & 9 \\
\hline $\begin{array}{c}\text { pachyonychia congenita type } \\
2\end{array}$ & Q04695 & P41219 & 9 \\
\hline $\begin{array}{c}\text { pachyonychia congenita type } \\
2\end{array}$ & Q04695 & P31947 & 9 \\
\hline $\begin{array}{c}\text { pachyonychia congenita type } \\
2\end{array}$ & Q04695 & P35900 & 9 \\
\hline $\begin{array}{c}\text { pachyonychia congenita type } \\
2\end{array}$ & Q04695 & P19012 & 9 \\
\hline
\end{tabular}




\begin{tabular}{|c|c|c|c|}
\hline $\begin{array}{c}\text { pachyonychia congenita type } \\
2\end{array}$ & Q04695 & Q6PH85 & 9 \\
\hline $\begin{array}{c}\text { pachyonychia congenita type } \\
2\end{array}$ & Q04695 & Q14CN4 & 9 \\
\hline $\begin{array}{c}\text { autoimmune } \\
\text { lymphoproliferative syndrome } \\
\text { type 1a }\end{array}$ & P25445 & P63165 & 22 \\
\hline $\begin{array}{c}\text { autoimmune } \\
\text { lymphoproliferative syndrome } \\
\text { type 1a }\end{array}$ & P25445 & Q14195 & 22 \\
\hline $\begin{array}{c}\text { autoimmune } \\
\text { lymphoproliferative syndrome } \\
\text { type 1a }\end{array}$ & P25445 & Q6EEV6 & 22 \\
\hline $\begin{array}{c}\text { autoimmune } \\
\text { lymphoproliferative syndrome } \\
\text { type 1a }\end{array}$ & P25445 & Q9UKR5 & 22 \\
\hline $\begin{array}{c}\text { autoimmune } \\
\text { lymphoproliferative syndrome } \\
\text { type 1a }\end{array}$ & P25445 & P52565 & \\
\hline
\end{tabular}

